\title{
Expanding Beyond the Micropore: Active-Site Engineering in Hierar- chical Architectures for Beckmann Rearrangement
}

\author{
Stephanie H. Newland, ${ }^{ \pm}$Wharton Sinkler, ${ }^{\S}$ Thomas Mezza,${ }^{\S}$ Simon R. Bare,${ }^{\S}$ Marina Carravetta, ${ }^{ \pm}$Ibra- \\ heem M. Haies, ${ }^{ \pm}$Alan Levy, ${ }^{\phi}$ Scott Keenan, ${ }^{\phi}$ Robert Raja ${ }^{ \pm *}$ \\ ${ }^{ \pm}$School of Chemistry, University of Southampton, Southampton SO17 1BJ, UK \\ ${ }^{\S}$ UOP, a Honeywell company, Des Plaines, IL 60017, USA \\ ${ }^{\phi}$ Honeywell Int., 101 Columbia Road, Morristown, NJ 0762, USA. \\ *R.Raja@soton.ac.uk
}

\begin{abstract}
The ability to engineer discrete solid-acid centers within hierarchically porous architectures that contain micropores with interconnected mesopores, offers the potential to overcome the spatial restraints and diffusional limitations imposed by conventional microporous zeotype catalysts, which often lead to decreased catalyst lifetimes and restricted substrate scope. By employing a one-step soft-templating approach, coupled with detailed physicochemical and spectroscopic characterization, isolated, solidacid sites can be suitably tailored and discretely modulated within the micropores and mesopores. The design strategy facilitates a synergistic enhancement in catalytic activity, selectivity, substrate versatility, and longevity, compared to analogous microporous zeotypes that have been extensively employed in the chemical industry, as solid-acid catalysts, for the production of $\varepsilon$-caprolactam (precursor for Nylon-6).
\end{abstract}

KEYWORDS: Solid-Acid Catalysis, Beckmann Rearrangement, Hierarchically Porous, Silicoaluminophosphates, Soft-templating,

\section{INTRODUCTION}

Microporous zeolites and zeotypes have proved to be exceptional solid-acid catalysts in the chemical industry, owing to their highly robust nature and tunable catalytic properties. It has been established that by careful consideration of the topology and composition of the zeotype, the nature and strength of (active) acid centers can be optimized for specific reactions, with high activities and selectivities. ${ }^{1}$ For example the Beckmann rearrangement of cyclic ketones relies upon very weak acid sites, therefore highly siliceous zeolites, such as ZSM-5 with silanol nests, are used within the chemical industry (Figure S1). ${ }^{2}$ In stark contrast, the methanol-to-olefin (MTO) process requires much stronger Brønsted acid sites, and zeotypes such as SAPO-34 (Figure S2 and S3) are better suited for this purpose. ${ }^{3}$ The ability to design and engineer isolated active sites (single-sites) at the molecular level has led to these catalysts being prolific within academia and industry, with advances in in situ and operando spectroscopic tools paving the way for establishing meaningful structureproperty correlations. ${ }^{4}$

Despite the widespread applicability of zeolites in industrial (acid-catalyzed) processes, ${ }^{5}$ their interconnecting micropores retard diffusion of organic molecules, which lowers reaction rates, increases coking and affords reduced substrate versatility. ${ }^{2,} 6$ To circumvent these limitations, mesoporous materials with adjustable larger pore sizes $\left(2-50 \mathrm{~nm}\right.$ diameter) such as M41S ${ }^{7}, \mathrm{FSM}-16^{8}$ and SBA- $n^{9}$ were developed. These materials proved somewhat effective in the functionalization of larger substrates for acidcatalyzed transformations; ${ }^{10}$ however their amorphous nature, coupled with their inferior stability and versatility, impeded their industrial implementation. More importantly, it was ascertained ${ }^{11}$

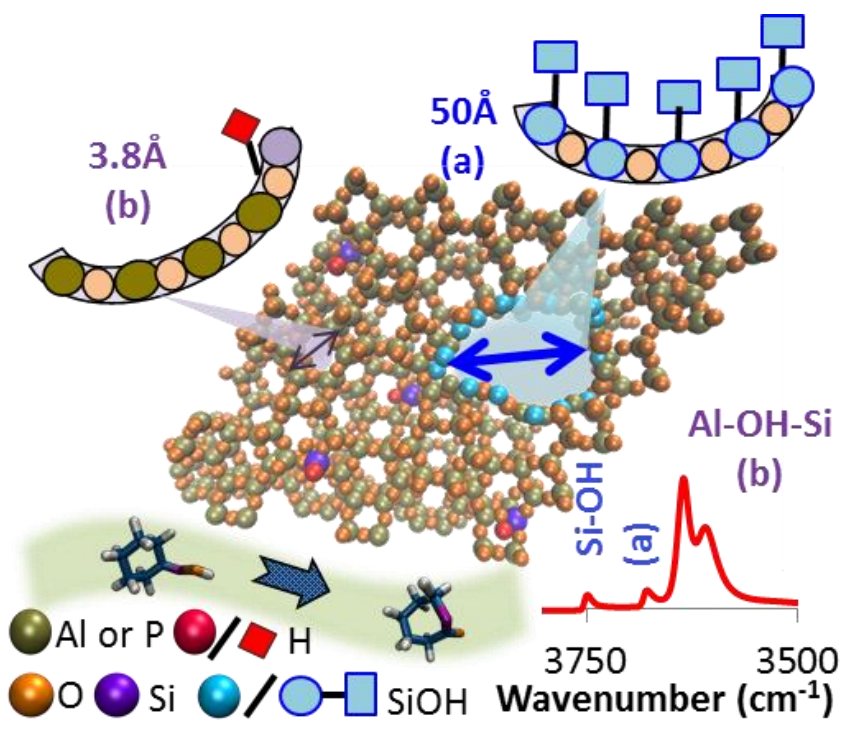

Figure 1. Graphical representation of a hierarchically porous SAPO-34 catalyst containing isolated silanol sites (a) within the mesopores and isolated Brønsted sites (b) in the micropores.

that the precise nature of the active (acid) centers in the mesoporous analogues was subtly different to that of their microporous 
counterparts (Figure S1-3), which resulted in much inferior catalytic activities and selectivities. Therefore, the notion of being able to combine the advantages of a microporous catalyst (welldefined isolated active sites) with the merits of a mesoporous system (more facile diffusion), in a hierarchically porous framework $^{12}$ is very attractive; provided one can mimic the precise nature (e.g. oxidation states, local coordination geometry, acid strength) of the active site at the molecular level, with a view to predicting and eliciting its catalytic function. ${ }^{1 \mathrm{~b}, 1 \mathrm{c}, 13}$

Herein we report our design-application approach for the creation of hierarchically porous (HP) SAPO-5 and SAPO-34 (Figure 1 and S4) catalysts, and their hitherto unreported, remarkable potential and performance in the industrially-significant Beckmann rearrangement to produce $\varepsilon$-caprolactam and other highervalue lactams. ${ }^{2}$ Specifically, our strategy relies upon being able to create analogous active sites, to the corresponding microporous counterparts, with an auxiliary mesoporous network to facilitate enhanced diffusion (mass-transport), which should directly influence the longevity and lifetime of the catalyst. Structural (XRD, HR TEM), textural (BET), spectroscopic (FT-IR, ssNMR) evidence is provided to corroborate the precise nature (and similarities) of the active (acid) site, with its microporous analogue, with a view to affording preliminary structure-property correlations, which could lead to the rational design of hierarchical architectures for a myriad of industrial applications. ${ }^{14}$

\section{EXPERIMENTAL}

\section{Material Preparation}

\section{Hierarchically Porous SAPO-5 Synthesis}

Aluminium isopropoxide (6.807g, Aldrich), phosphoric acid (2.28ml, $85 \%$ in $\mathrm{H}_{2} \mathrm{O}$, Aldrich) and water $(10 \mathrm{ml})$ were vigorously stirred until homogeneous. Dimethyloctadecyl[(3(trimethoxysilyl)propyl] ammonium chloride, DMOD, $(1.2 \mathrm{ml}$, $72 \%$ in $\mathrm{H}_{2} \mathrm{O}$, Aldrich) was added drop wise, followed immediately by addition of triethylamine $(3.7 \mathrm{ml}$, Aldrich) and water $(20 \mathrm{ml})$. After stirring for one hour, silica sol $(0.771 \mathrm{ml}, 40 \%$ in water, Aldrich) was added and the gel was stirred for a further 1.5 hours to obtain a white gel with the composition:

\section{$1.0 \mathrm{Al}: 1.0 \mathrm{P}: 0.8$ TEA: $50 \mathrm{H} 2 \mathrm{O}: 0.15 \mathrm{Si}$ : 0.05 DMOD.}

The gel was transferred to a Teflon-lined stainless-steel autoclave, which was set to crystallize in a pre-heated fan assisted oven (WF-30 Lenton) at $200^{\circ} \mathrm{C}$ for 24 hours. The white solid product from the autoclave was collected via filtration and washed with $500 \mathrm{ml}$ of deionized water. The product was left to dry at $80^{\circ} \mathrm{C}$ overnight. The as-synthesized catalyst was calcined in a tube furnace under a flow of air at $550^{\circ} \mathrm{C}$ for 16 hours to produce a white crystalline solid.

An equivalent method was deployed for the synthesis of the microporous analogue without the inclusion of DMOD.

\section{Hierarchically Porous SAPO-34 Synthesis}

Aluminium isopropoxide $(4.5450 \mathrm{~g}$, Aldrich) was added to tetraethyl ammonium hydroxide (TEAOH) $\left(9.14 \mathrm{ml}, 35 \%\right.$ in $\mathrm{H}_{2} \mathrm{O}$, Aldrich) and stirred for one hour. Fumed silica (0.2g) and DMOD $(0.8 \mathrm{ml}, 72 \%$ in water, Aldrich) were added to the synthesis and stirred until homogeneous. Deionized water $(14 \mathrm{ml})$ was added drop wise followed directly by phosphoric acid $(1.5 \mathrm{ml}, 85 \%$ in $\mathrm{H}_{2} \mathrm{O}$, Aldrich). The gel was vigorously stirred for two hours to produce a white gel with the composition:

\subsection{Al: 1.0 P: 1 TEAOH: 65 H2O: 0.15 Si: 0.05 DMOD.}

The gel was transferred to a Teflon-lined stainless-steel autoclave, which was set to crystallize in a pre-heated fan assisted oven (WF-30 Lenton) at $200^{\circ} \mathrm{C}$ for 48 hours. The white solid product from the autoclave was collected via filtration and washed with $500 \mathrm{ml}$ of deionized water. The product was left to dry at $80^{\circ} \mathrm{C}$ overnight. The as-synthesized catalyst was calcined in a tube furnace under a flow of air at $550^{\circ} \mathrm{C}$ for 16 hours to produce a white crystalline solid.

An equivalent method was deployed for the synthesis of the microporous analogue without the inclusion of DMOD.

\section{Characterization}

All powder X-ray diffraction patterns were collected using a Bruker D2 diffractometer using $\mathrm{Cu} \mathrm{K} \alpha 1$ radiation. Low-angle $\mathrm{X}$ ray diffraction patterns were obtained using a Bruker C2 GADDS diffractometer. BET surface area measurements were performed using a Micromeritics Gemini 2375 surface area analyzer. FT-IR spectra of the pelletized samples were recorded with a Nicolet Nexus 870 IR Spectrometer for 128 scans using a cooled MCT detector. All spectra were processed using the GRAMS/Al 9 software (Thermo Scientific.). Low-temperature CO adsorption was performed by sequentially added $0.02 \mathrm{cc}$ of $\mathrm{CO}$ and allowing to equilibrate for 3 minutes after each injection before recording the spectrum. Collidine adsorption was performed by flowing helium saturated with colliding at $7^{\circ} \mathrm{C}$ over the sample for one hour at $150^{\circ} \mathrm{C}$. Stepwise desorption at 150,300 and $450^{\circ} \mathrm{C}$ was then preformed in flowing helium for one hour at each temperature.

TPD measurements were performed on a custom-built system using TCD detectors to monitor ammonia concentration. Samples were pre-treated by heating at $10^{\circ} \mathrm{C} / \mathrm{min}$ to $550^{\circ} \mathrm{C}$ in a $20 \%$ $\mathrm{O}_{2}$ /helium mixture for 2 hours. The samples were exposed to ammonia and allowed to equilibrate at $150^{\circ} \mathrm{C}$ for 8 hours. Desorption was performed in flowing at $10^{\circ} \mathrm{C} / \mathrm{min}$ to $600^{\circ} \mathrm{C}$ and held for 40 minutes at $600^{\circ} \mathrm{C}$. TGA (TA instrument Q50) were performed under nitrogen with a $20^{\circ} \mathrm{C}$ per minute ramp rate. Samples were dissolved in a $\mathrm{HCl}$ solution before being aspirated into a PerkinElmer Optima 8300 ICP-OES instrument for ICP analysis. Scanning electron microscopy images were obtained using a JSM5900 LV SEM and Transmission electron microscopy images were obtained with a JEM-3000F instrument.

Solid State NMR: all spectra were acquired using a Bruker AVANCE II AscendTM $400 \mathrm{WB}$ magnet on a Chemagnetics Infinity console at $9.4 \mathrm{~T}$. All the NMR experiments were performed on a $6 \mathrm{~mm}$ double-resonance APEX probe. Approximately $150 \mathrm{mg}$ of material was transferred to a normal wall zirconium oxide rotor and spun at $6 \mathrm{kHz}$. The ${ }^{29} \mathrm{Si}$ peak position was referenced to silicon rubber as a secondary reference at $-22.3 \mathrm{ppm}$ with respect to TMS. ${ }^{15}{ }^{29} \mathrm{Si}$ spectra were obtained using ramped crosspolarization with a contact time $5 \mathrm{~ms}$ and an amplitude ramp of $5 \%,{ }^{1} \mathrm{H}$ SPINAL-64 decoupling was used during acquisition. ${ }^{16}$ The $2 \mathrm{D}{ }^{29} \mathrm{Si}$ spectra were recorded using proton-driven spin diffusion methods ${ }^{17}$ with a mixing time of $5 \mathrm{~ms} .{ }^{31} \mathrm{P}$ spectra were acquired using single-pulse acquisition and the spectra were referenced with respect to $85 \% \mathrm{H}_{3} \mathrm{PO}_{4} .{ }^{27} \mathrm{Al}$ were referenced with respect to a $1 \mathrm{M} \mathrm{Al}\left(\mathrm{NO}_{3}\right)_{3}$ solution $(0 \mathrm{ppm})$. All spectra were processed using matNMR ${ }^{18}$ with Matlab2014b.

\section{Catalysis}

A cylindrical quartz fixed-bed reactor ( $4 \mathrm{~mm}$ in diameter) with a quartz frit was packed with $0.5 \mathrm{~cm}$ layer of glass beads $(1 \mathrm{~mm})$. The catalyst $(0.2 \mathrm{~g})$ was pelletized and added to the reactor creating a $4 \mathrm{~cm}$ layer. A further $20 \mathrm{~cm}$ of glass beads $(1 \mathrm{~mm})$ were placed inside the heater unit of the reactor assembly. The sample was then pre-treated at $673 \mathrm{~K}$ under a $50 \mathrm{ml} / \mathrm{min}$ flow of helium gas for one hour. The temperature was then lowered to $598 \mathrm{~K}$ and the flow of helium was reduced to $33.3 \mathrm{ml} / \mathrm{min}$. A liquid-feed of $100 \mathrm{~g} / \mathrm{liter}$ of cyclohexanone oxime in ethanol was fed, via elec- 
tronic syringe pump, into the reactor to maintain a WHSV of $0.79 \mathrm{hr}^{-1}$ that was controlled by an electronic syringe pump. Samples were analyzed after every hour, when steady-state was achieved, using a Clarus 480 gas chromatograph with FID detector and using an Elite 5 column. Products were identified against authenticated standards and quantified by calibration to obtain response factors (RF) against the known internal standard (mesitylene).

An identical protocol was followed for the vapor-phase Beckmann rearrangement of cyclooctanone oxime.

\section{RESULTS AND DISCUSSION}

The HP SAPOs reported herein were synthesized by utilizing a one-pot soft-templating strategy in which an organosilane surfactant, ${ }^{19}$ dimethyloctadecyl[(3-(trimethoxysilyl)propyl] ammonium chloride (DMOD), was used in combination with the microporous structure-directing agent (TEA, TEAOH). It was hypothesized that the siliceous-component of the surfactant would be incorporated within the SAPO framework (TGA (S5-S8)), and upon removal of the organic hydrophobic tail, by calcination in air, additional (further to type II and type III substituted Brønsted-acid sites - see Figures S2 and S4) silanol sites would be generated. ${ }^{20}$ It is envisaged that these acidic silanol sites would then participate in the Beckmann rearrangement, in an analogous fashion to the defect sites found in highly siliceous zeolites; ${ }^{2}$ but with the added advantage of having the Brønsted-acid sites generated at the micropores to supplement and boost the catalytic potential (bifunctional catalysis). To effectively investigate our hypothesis, microporous analogues of SAPO-5 and SAPO-34 were also synthesized with comparable amounts of Si. The phase-purity and crystallinity of all materials were confirmed via powder X-ray diffraction (see Figure 2 and Figure S9 for patterns and Table S2-5 for associated Reitveld refinements). Low-angle XRD measurements of the hierarchical samples revealed a broad-peak at low-angles ( $<5 \operatorname{deg} 2-\theta$, see inset), which was absent in the microporous samples, indicating the presence of mesopores.

To further substantiate the efficacy of our design strategy to generate hierarchical catalysts, BET (Figure 2, Figure S10 and Table S6) measurements were performed. Encouragingly, all the hierarchical samples exhibited type IV isotherms (Figure 2 and Figure S10) with hysteresis, which is indicative of the presence of mesopores. The micropore and mesopore volumes were evaluated (Table S6) and it was noteworthy that, while the micropore volumes were similar, the resulting surface areas and mesopore volumes for both hierarchical catalysts were substantially higher when compared with their microporous counterparts. Additionally, the BJH pore distribution curves (Figure 2 and S10) further confirm the presence of mesopores within the hierarchical systems, which strongly indicates the successful incorporation of mesopores into the hierarchical frameworks (Table S6).

The porosity of the hierarchical catalysts was further evaluated via both SEM, with EDXS (Figure S11-S16), and HR TEM (Figure 3 and S17-20). The SEM and EDXS shows that both HP samples have a fairly uniform composition throughout, while the TEM study complemented these findings (see figures S11-20), in addition to revealing the true-mesoporosity in both of the crystalline HP SAPOs. From the TEM and associated selected-area electron diffraction patterns of HP SAPO-34, it was possible to elucidate the rod-like and elongated shapes of the mesopores and their positioning perpendicular and parallel to the rhombohedral basis vectors. It was also remarkable that these mesopores were wellconnected within the microporous network. (Figure 3 and S19-20)

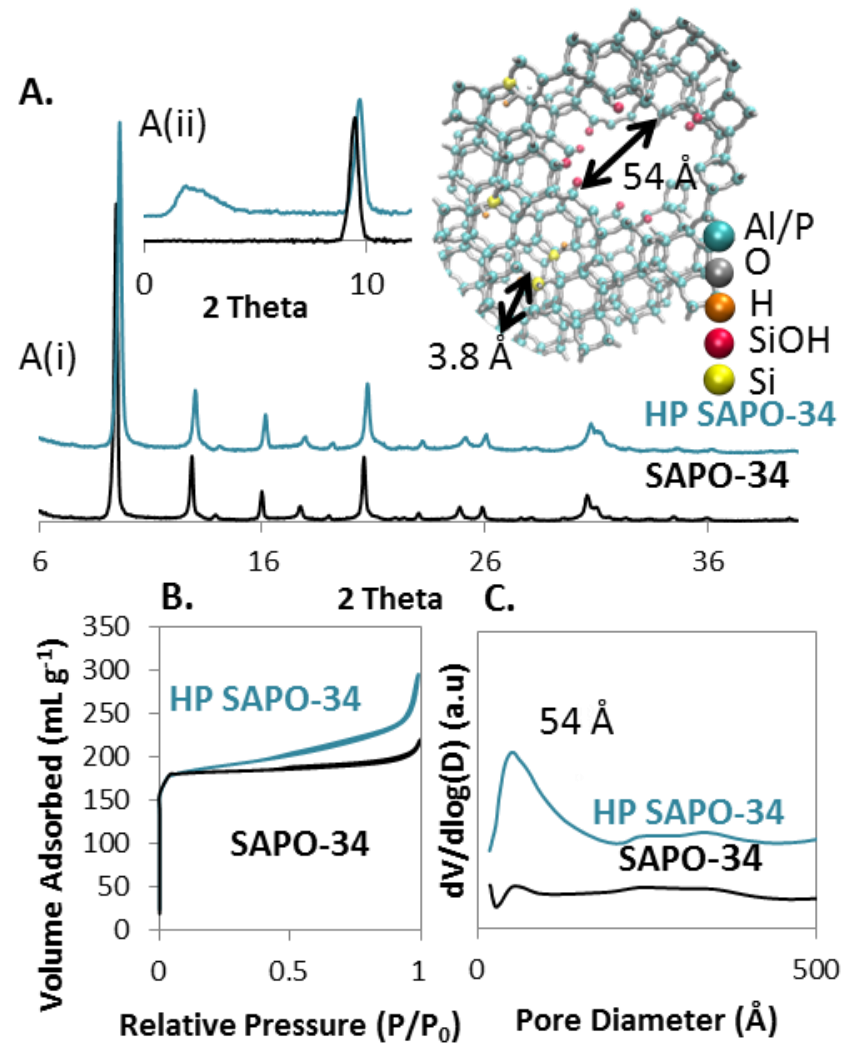

Figure 2. Powder XRD pattern of microporous SAPO-34 (black) and HP SAPO-34 (blue) (A (i)) with low-angle pattern insert (A (ii)). Nitrogen adsorption isotherm (B) and BJH pore distributions (C).

Given these promising characterization data, the catalytic potential for the hierarchical samples was assessed in the vapor-phase Beckmann rearrangement of cyclohexanone oxime (page S19) to $\varepsilon$-caprolactam (the industrially-significant precursor to Nylon-6) and direct comparisons were made to the corresponding microporous analogues. The difference in catalytic performance between the two systems was striking: the hierarchical catalysts were superior and maintained a constant reactivity and selectivity (Figure 4; see also Figure S24-26), while in stark contrast, the microporous analogues, as well as the commercial prototype, zeolite H-ZSM-5, deactivated progressively with time-on-stream.

In order to verify that the improved catalytic performance of the HP catalysts is not purely due to better diffusion ${ }^{12}$ afforded by the larger mesopores within the HP architecture, we contrasted the catalytic performance using a well-established ${ }^{7}$ ordered mesoporous silica (MCM-41), containing pendant silanol groups that have proved effective in acid-catalyzed transformations. ${ }^{2} \mathrm{We}$ also deliberately synthesized a HP AlPO-5 catalyst, which is not expected to contain any isomorphously substituted ( $\mathrm{Si}(\mathrm{IV})$ ) Brønsted acid centers, but primarily possesses silanol sites (blue circle and square, Figure 1), due to the synthetic strategy that involves the use of DMOD (see Experimental, page 2). Both these catalysts were much inferior in performance (both activity and selectivity) for the Beckmann rearrangement. After 7 hours, HP AlPO-5 had lower conversion compared to HP SAPO-5 (69\% versus >98\%) for the vapor-phase Beckmann rearrangement of cyclohexanone oxime and it also displayed lower selectivity (62\% versus $77 \%$ ). MCM-41, on the other hand, also had much lower conversion (23\%) and selectivity (11\%), in comparison to the AFI frameworks (Figure 4). Both of these catalysts do not have isolated 
Brønsted acid sites (red diamond, Figure 1) and did not perform as well as the HP SAPO-5 (or HP SAPO-34), that contain the additional Brønsted acid sites. Therefore we believe there is a clear interplay between these two sites, which results in the improvements seen in the catalysis. This further highlights the importance of adroitly engineering the precise active site within a stable porous framework, with the dual benefit of enhancing diffusion and mass-transfer via an auxiliary mesoporous network.

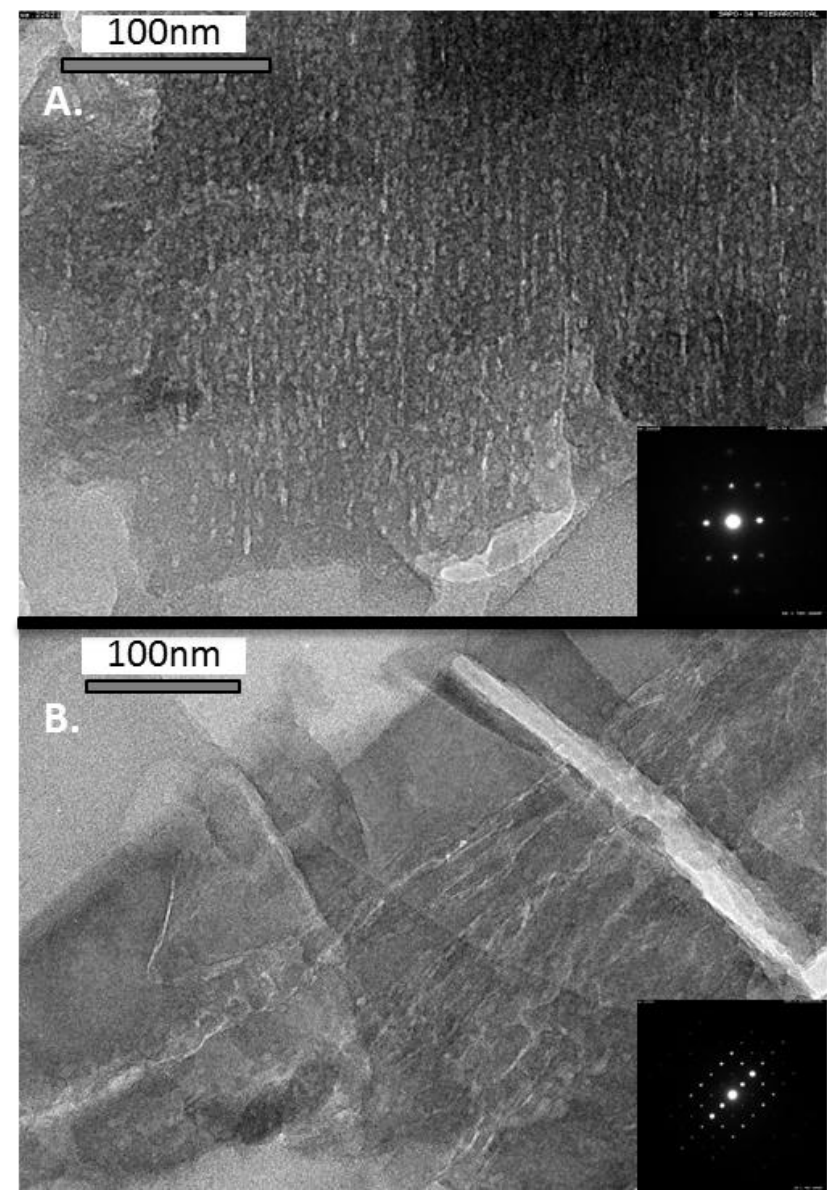

Figure 3. Structural (HR TEM and selected-area electron diffraction) evidence, substantiating the structural integrity, of (A) HP SAPO-34 (CHA) and (B) HP SAPO-5 (AFI), and the presence of hierarchical (dual) porosity of the catalysts.

It is clear that the hierarchical catalysts can sustain their high catalytic performance and have improved longevity in the Beckmann rearrangement, which suggests minimal coke formation with time-on-stream. Coking can occur if the acid sites are too strong and therefore do not permit the ready desorption of the product; or it can occur if diffusion is hindered, preventing the egress of products. Therefore, to further establish the origin of the enhanced catalytic performance of the HP catalysts, in-depth characterization of the nature and strength of the solid-acid centers (using ssNMR, TPD of $\mathrm{NH}_{3}$, and FTIR using adsorbed $\mathrm{CO}$ at $100 \mathrm{~K}$ and FTIR of adsorbed 2,4,6-trimethylpyridine (collidine) as molecular probes) were performed.

MAS NMR spectroscopy was deployed to investigate the nature (local coordination geometry) of the $\mathrm{Al}, \mathrm{P}$ and $\mathrm{Si}$ sites within the hierarchical frameworks (Figure 5 and S27-30). ${ }^{29}$ Si MAS and 2D NMR (Figure 5) of the two hierarchically porous catalysts was very revealing: both samples contained isolated silicon sites (formed via type II substitution see Figure S2), as well as silicon islands, which are more likely to be generated via both type III substitution and through the incorporation of the surfactant into the framework (as described earlier).

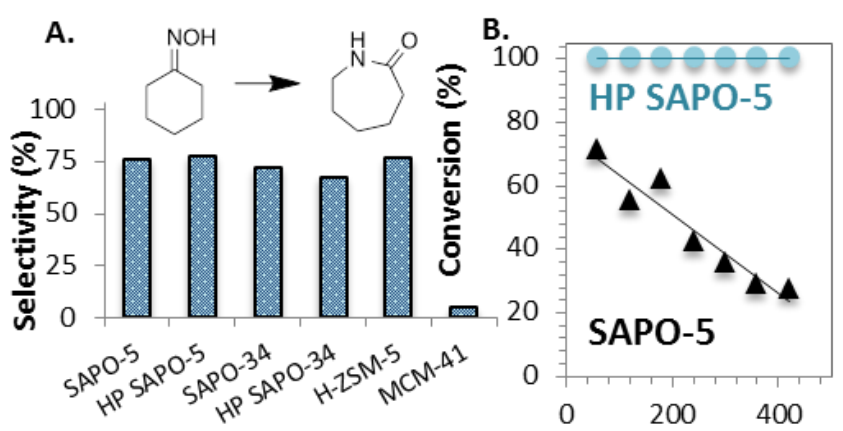

C.

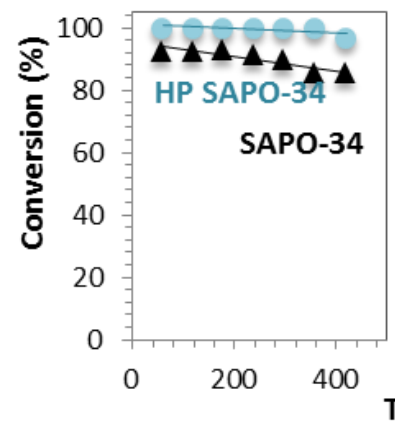

D.

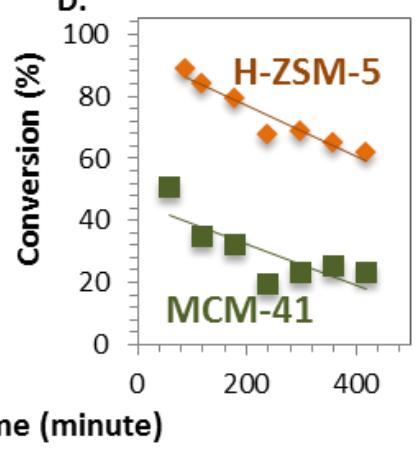

Figure 4. Comparison of catalytic performance ((B) HP SAPO-5 and SAPO-5, (C) HP SAPO-34 and SAPO-34, and (D) H-ZSM-5 and MCM-41) in the vapor-phase Beckmann rearrangement of cyclohexanone oxime. The selectivity to $\varepsilon$-caprolactam (1h) is shown in (A) at $598 \mathrm{~K}$ with $0.79 \mathrm{hr}^{-1}$ WHSV.

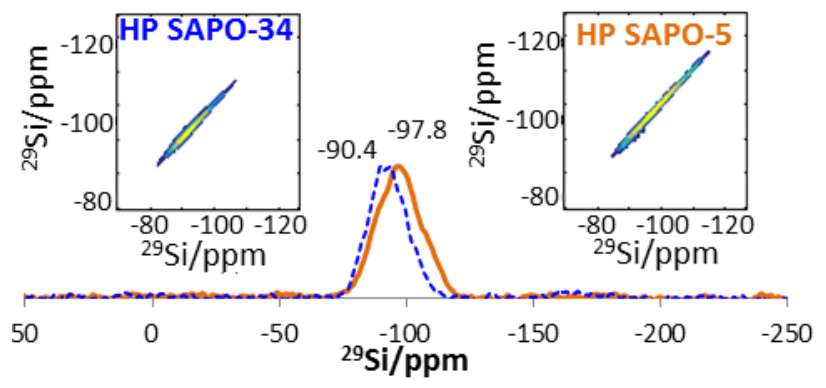

Figure 5. ${ }^{29} \mathrm{Si}$ MAS NMR of HP SAPO-5 and HP SAPO-34 and ${ }^{29} \mathrm{Si} 2 \mathrm{D}$ homonuclear correlation spectrum acquired at $400 \mathrm{MHz}$ under MAS with $6 \mathrm{kHz}$ spinning frequency for HP SAPO-34 (left) and HP SAPO-5 (right).

The acid strength of these materials will be intimately related to the ensuing catalytic properties; hence it will be meaningful to investigate both strength and type of acidity, in order to facilitate in-depth comparisons. Thus the nature and strength of the acid centers in the hierarchical catalysts were probed using FT-IR, employing $\mathrm{CO}$ and collidine as molecular probes. First, a comparison of the hydroxyl stretching region in the HP SAPO-34 and HP SAPO-5 samples is shown in Figure 6A (and S31-34). Both catalysts show IR bands attributable to P-OH/Al-OH $\left(3678 \mathrm{~cm}^{-1}\right)$ defect sites and bands $\left(3628-3600 \mathrm{~cm}^{-1}\right)$ arising from the substitution of silicon into the framework ( $\mathrm{Si}-\mathrm{OH}-\mathrm{Al})$. Notably within the 
HP SAPO-34 the FTIR revealed that there is a considerable higher quantity of Brønsted acid sites in comparison to HP SAPO-5. Despite this, HP SAPO-5 displays nearly $100 \%$ conversion of cyclohexanone oxime in the vapor phase reaction (Figure 4) as does HP SAPO-34. The FTIR spectra of both catalysts also revealed an additional band at $3746 \mathrm{~cm}^{-1}$ of similar intensity that is assigned to $\mathrm{Si}-\mathrm{OH}$ groups, which were marginal in the FT-IR of the microporous ${ }^{21}$ catalysts, indicating that these silanol sites were formed via the calcination of the surfactant, as discussed earlier. These results suggest that the $\mathrm{Si}-\mathrm{OH}$ groups play a key role in the high conversion of the HP SAPOs (Figure 4). These silanol groups are particularly significant, as analogous sites in zeolites and other zeotypes ${ }^{2,3}$ have been shown to be highly active and proficient for the Beckmann rearrangement, and arguably better suited than Brønsted acid sites (Scheme 1). ${ }^{22}$ The more acidic Brønsted acid sites can lead to the basic lactam product becoming protonated and strongly adsorbed on the catalyst surface, resulting in blocking of active sites, coupled with the prospect of consecutive undesired reactions, which lead to reduced selectivity and catalyst deactivation. Therefore the $\mathrm{Si}-\mathrm{OH}$ sites are considered to be more amenable for the Beckmann rearrangement and it is these sites that were considered responsible for the extremely high conversions and selectivities with the industrial catalyst, Silicate-1.2, $22 \mathrm{c}, 23$

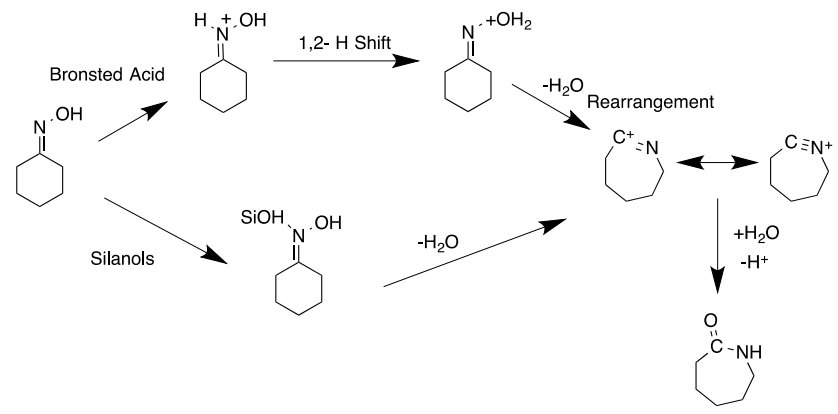

Scheme 1. The role of acidic silanols and Brønsted acid sites in the Beckmann rearrangement of cyclohexanone oxime. ${ }^{2 a}$

Notably, TGA data (Figure S35-S38) of the used catalysts revealed similar (minimal) levels of carbon on both the microporous and hierarchically porous materials. From the catalysis data (Figure 4) it is clear that the hierarchically porous catalysts were not affected by the coking, in stark contrast to the microporous analogues. Therefore, this suggests that the coke is preferentially adsorbing onto the Brønsted acid sites that are located primarily within the micropores in both the microporous and hierarchically porous catalysts (Figure 6A). This leads to the progressive deactivation of the microporous catalysts in the Beckmann rearrangement (Figures 4 and 7), while the additional silanol sites, (that are more resistant to coking), which are located within the mesopores, confer enhanced lifetimes to the hierarchically-porous catalysts. In addition to these observations, we note that our mass-balance was consistently greater that $97 \%$ (see pages S20-21, S30-31), which further vindicates our assertion on minimal coke formation.

FT-IR with $\mathrm{CO}$ adsorbed at low-temperature elucidated that, in both HP SAPO-5 and HP SAPO-34 no visible bands that can be attributable to Lewis acidity were detected - this was conspicuous by an absence of a characteristic absorption $\geq 2190 \mathrm{~cm}^{-1}$ (Figure 6C and 6D, S31 and S32). Instead, only signals associated with Brønsted acid sites were detected, which is consistent with previously observed data with the microporous analogues. ${ }^{21}$ Evaluation of the band-shift of the Brønsted acid sites between $268-286 \mathrm{~cm}^{-1}$, upon interaction with $\mathrm{CO}$ (Table 1) revealed that, both samples primarily consisted of moderate-strength Brønsted acid sites. By integrating the area of the Brønsted acid peaks it was possible to establish that the HP SAPO-34 catalyst has a greater proportion of total Brønsted acidity, when compared with the HP SAPO-5 sample. This trend is in good agreement with the temperatureprogrammed desorption results using $\mathrm{NH}_{3}$ as the molecular probe (Figure 6B and S39). Despite HP SAPO-5 having much lower total acidity and Brønsted acidity than HP SAPO-34 it is still able to reach comparable conversions of $100 \%$ (Figure 4). However, the $\mathrm{Si}-\mathrm{OH}$ sites appear to be in similar concentrations in the HP SAPO-5 and HP SAPO-34, which strongly suggests that the Si$\mathrm{OH}$ sites within the mesopores are primarily responsible for the high conversions in the hierarchically porous catalysts.

Interestingly, and more importantly, the acid-strength of both hierarchical catalysts was comparable with the previously reported microporous analogues (Table 1; see also S39), which emphasizes the fact, using our soft-templating design approach, that it is possible to mimic the precise nature and strength of the active sites within both the microporous and hierarchical framework architectures.
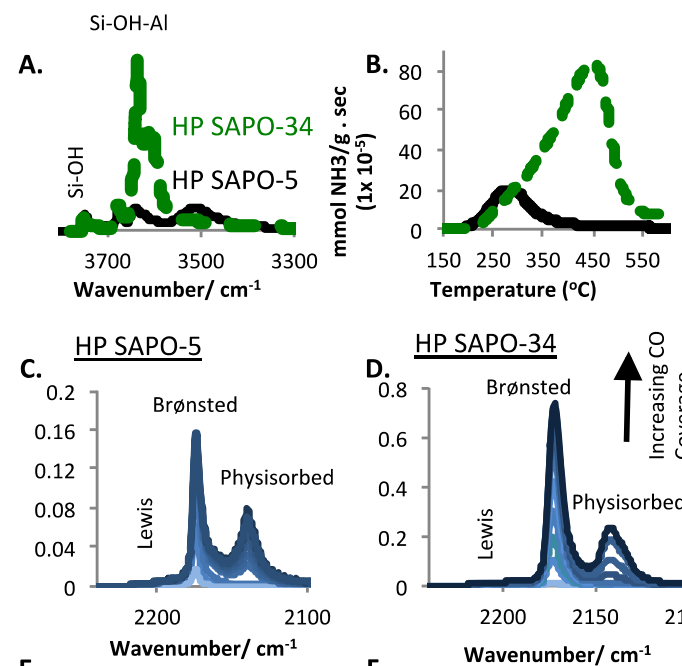

E.

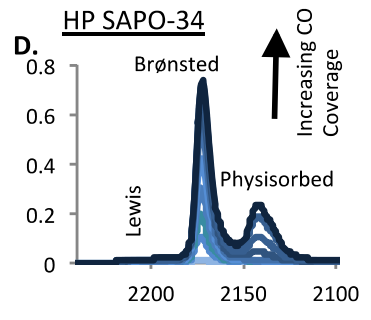

F.

Wavenumber $/ \mathrm{cm}^{-1}$

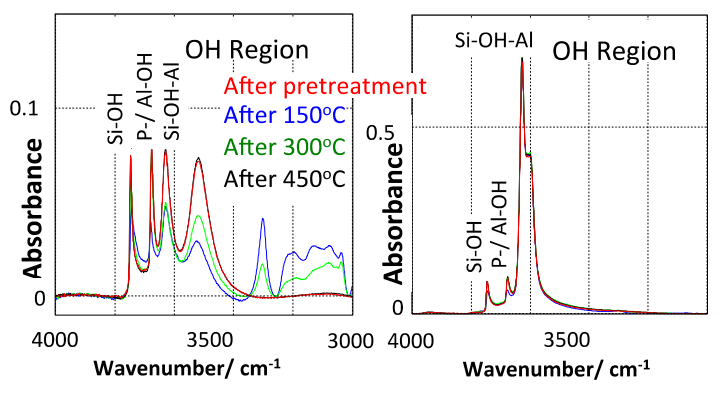

Figure 6. (A) FTIR spectra of hydroxyl region of calcined HP SAPO-34 (dotted green) and HP SAPO-5 (black). (B) TPD-NH adsorption data of HP SAPO-34 (dotted green) and HP SAPO-5 (black). (C \& D) FTIR spectra of CO adsorbed (0.02cc at 100K) at low temperatures on calcined HP SAPO-5 and HP SAPO-34 (respectively). (E \& F) FTIR spectra of collidine adsorbed on calcined HP SAPO-5 and HP SAPO-34 respectively.

In order to further explore the nature and accessibility of the acid sites within the hierarchical SAPOs, a complementary probe, collidine, was employed. The corresponding FTIR spectra on HP SAPO-34 and HP SAPO-5 (Figure 6E and 6F, S33 and S34) clearly indicated that the acid strength on the hierarchical structures is comparable to the previously reported microporous catalysts. ${ }^{2,3}$ In both cases, all the collidine is desorbed by $450^{\circ} \mathrm{C}$. Analogous to the FTIR-CO results, the collidine mainly adsorbs onto the moderate-to-weak acid sites, including the silanol groups, 
which are present in the mesopores. The latter are readily available to interact with the substrates, thereby enhancing the catalysts' potential in the Beckmann rearrangement.

Given their propensity for modulating and mimicking the nature and strength of acid sites (in sync with their microporous counterparts $^{1 \mathrm{~b}, 1 \mathrm{c}}$ ), the versatility and stability of the hierarchically porous catalysts was further demonstrated in the vapor-phase Beckmann rearrangement of the more sterically-demanding cyclooctanone oxime (Figure 7 and S40). It was again noteworthy that the hierarchical catalysts revealed a prodigious catalytic performance (whilst retaining their structural integrity (Figure S41 and S42)), whereas their microporous counterparts exhibited inferior activities and diminished lifetimes. Interestingly, the catalytic activity of the microporous SAPO-34 and SAPO-5 is greatly reduced (c.f. Figure 4) when the bulky cyclooctanone oxime is used as the substrate, which further substantiates the location of active (acid) sites within the micropores, and highlights the importance of shape-selectivity (spatial restraints) and diffusion-control in the ensuing catalysis.

Table 1. Position of maxima of the $\mathrm{OH}$ stretching frequency of the Brønsted sites and their shifts $\left(\Delta v_{\mathrm{OH}}\right)$ upon CO Adsorption at $100 \mathrm{~K}$ on HP SAPO-34, HP SAPO-5, and their corresponding microporous analogues.

\begin{tabular}{|c|c|c|c|c|}
\hline \multicolumn{2}{|l|}{ Catalysts } & \multirow{2}{*}{$\begin{array}{l}\mathrm{VOH}\left(\mathrm{cm}^{-1}\right) \\
3638\end{array}$} & \multirow{2}{*}{$\begin{array}{l}\text { VOH-CO }\left(\mathrm{cm}^{-1}\right) \\
3368\end{array}$} & \multirow{2}{*}{$\begin{array}{l}\Delta \mathrm{VOH}\left(\mathrm{cm}^{-1}\right) \\
270\end{array}$} \\
\hline SAPO-5 & $\mathrm{OH}_{\mathrm{A}}$ & & & \\
\hline HP SAPO-5 & $\mathrm{OH}_{\mathrm{A}}$ & 3637 & 3369 & 268 \\
\hline SAPO-34 & $\mathrm{OH}_{\mathrm{A}}$ & 3633 & 3347 & 286 \\
\hline & $\mathrm{OH}_{\mathrm{B}}$ & 3610 & 3281 & 329 \\
\hline \multirow[t]{2}{*}{ HP SAPO-34 } & $\mathrm{OH}_{\mathrm{A}}$ & 3633 & 3347 & 286 \\
\hline & $\mathrm{OH}_{\mathrm{B}}$ & 3612 & 3281 & 331 \\
\hline
\end{tabular}

Further comparison of Figure 4C and Figure 7C reveals that the conversion of the cyclic oxime over the microporous SAPO-34 is greatly reduced ( $90 \%$ to about $40 \%$ ) as the oxime size increases; whereas the HP SAPO-34 analogue retains almost $100 \%$ conversion, regardless of the cyclic oxime size. Interestingly, despite FTIR-CO (Figure 6D) revealing that HP SAPO-34 has more total Brønsted acid sites than HP SAPO-5, the FTIR collidine (Figure $6 \mathrm{E}$ and F) data illustrated that the amount of (accessible) acid sites in HP SAPO-34 that can interact with collidine is much smaller

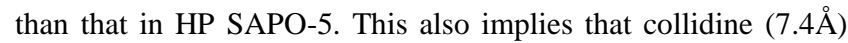
probably would not be able to access the Brønsted acid sites within microporous SAPO-34 (3.8 $)$ catalyst, and hence the activity of SAPO-34 is much lower in the Beckmann rearrangement of cyclooctanone oxime. Strikingly HP SAPO-34 has approximately $100 \%$ conversion of cyclooctanone oxime, despite the difficulties in the substrate access (as observed via FTIR-collidine adsorption to the Brønsted active sites within the micropores). Therefore it is highly plausible that the conversion of cyclooctanone oxime originates purely from the $\mathrm{Si}-\mathrm{OH}$ groups found the mesopores of the hierarchically porous catalysts (Figure 1). However it should be noted that HP AlPO-5 (see page 3) had a much lower reactivity was quickly deactivated, having just $46 \%$ conversion of cylcooctanone oxime at the end of the reaction, similar to MCM41. This further illustrates that there is a clear interplay between the silanol sites (in the mesopores) and the isolated Brønsted acid sites that originate from the isomorphous substitution of $\mathrm{Si}(\mathrm{IV})$ into the framework. It has been suitably demonstrated that a combination of these bifunctional active centers coupled with the secondary porosity of the hierarchically architectures confers a de- gree of multifunctionality that is responsible for the remarkable catalysis observed with the HP SAPOs (Figure 4 and Figure 7).

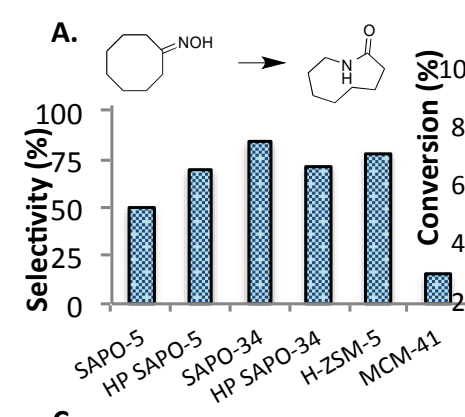

B.
C.

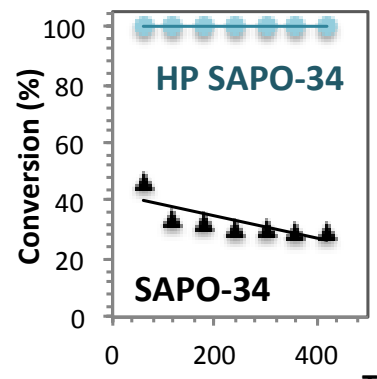

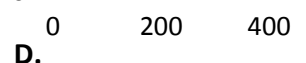

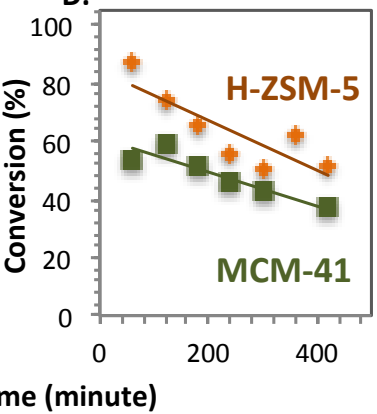

Figure 7. Comparison of catalytic performance ((B) HP SAPO-5 and SAPO-5, (C) HP SAPO-34 and SAPO-34, and (D) H-ZSM-5 and MCM-41) in the vapor-phase Beckmann rearrangement of cyclooctanone oxime. The selectivity to caprylic lactam (after 1h) is shown in (A) at $598 \mathrm{~K}$ with $0.79 \mathrm{hr}^{-1}$ WHSV.

\section{SUMMARY AND FUTURE PROSPECTS}

It is well-documented ${ }^{22 a, 24}$ that both weakly acidic silanols and isolated weak Brønsted acid sites are active in the vapor phase Beckmann rearrangement, with the consensus largely being that silanol nests are the preferential active site. By utilizing a one-pot soft silyl- surfactant strategy we have shown (via FTIR-CO, FTIR-collidine, MAS/NMR, TPD) that it is possible to create HP SAPO-5 and HP SAPO-34 architectures, which contain both isolated weak Brønsted acid sites within the micropores and silanol sites within the mesopores. The weaker acid sites within these hierarchical architectures facilitate the desorption of the basic lactam from the active site, thereby preventing consecutive reactions and poisoning of the catalyst. Therefore in the HP SAPOs it is highly likely that both the Brønsted acid centers and acidic silanols are the catalytic active sites for the Beckmann rearrangement (Scheme 1). The interplay between these two sites could be directly responsible for the enhanced catalytic performance of the hierarchical catalysts or the presence of these discrete acid centers could influence the synthesis to produce modulated active sites that are highly conducive for the Beckmann rearrangement. These resulting bifunctional hierarchically porous catalysts have improved lifetimes and enhanced substrate scope, compared to their microporous counterparts, in vapor-phase Beckmann rearrangements.

It has been demonstrated that the inclusion of auxiliary mesopores (in the HP SAPO-5 and HP SAPO-34) crucially increases the accessibility of the active sites towards the bulky substrates, and advantageously, the presence of the additional silanol groups in the hierarchical catalysts further modulates the acidity and tailors the acid-strength, thereby enhancing its catalytic performance in the Beckmann rearrangement. More importantly, our design 
approach has demonstrated that the precise nature and strength of the catalytic active centers in microporous SAPOs can be effectively mimicked in their hierarchical counterparts, which makes the latter set of catalysts (c.f. Figure 7D) more amenable for industrial application, given their enhanced longevity and stability.

In summary, the ability to adroitly engineer and mimic the precise nature and strength of solid-acid sites within hierarchical architectures ${ }^{14}$ offers significant benefits in improving the catalytic performance, substrate versatility, and overall durability, compared to analogous microporous systems, in industriallysignificant Beckmann rearrangement reactions. Creating an axillary mesoporous network, in close proximity to the micropores enhances diffusion and mass-transport, with the added benefit of modulating and tailoring the acid strength for eliciting superior and stable catalytic performance.

\section{ASSOCIATED CONTENT}

Supporting Information. Experimental details, additional characterization and catalysis data and analytical protocols. This material is available free of charge via the Internet at http://pubs.acs.org.

\section{Corresponding Author}

*R.Raja@ soton.ac.uk

\section{ACKNOWLEDGMENT}

We acknowledge Honeywell LLC for financial support. UK Catalysis Hub is thanked for resources and support provided via our membership of the UK Catalysis Hub Consortium and funded by EPSRC (EP/K014714/1). MC acknowledges the Royal Society University Research Fellowship scheme.

\section{REFERENCES}

1. (a) Raja, R.; Potter, M. E.; Newland, S. H., Chem. Commun. 2014, 50, 5940-5957; (b) Potter, M. E.; Sun, D.; Gianotti, E.; Manzoli, M.; Raja, R., Phys. Chem. Chem. Phys. 2013, 15, 1328813295; (c) Gianotti, E.; Manzoli, M.; Potter, M. E.; Shetti, V. N.; Sun, D.; Paterson, A. J.; Mezza, T. M.; Levy, A.; Raja, R., Chem. Sci. 2014, (5), 1810-1819; (d) Newland, S. H.; Xuereb, D. J.; Marchese, L.; Rios, R.; Raja, R., Catal. Sci. Technol. 2015, 5, 660-665.

2. Izumi, Y.; Ichihashi, H.; Shimazu, Y.; Kitamura, M.; Sato, H., Process Bull. Chem. Soc. Jpn 2007, 80 (7), 1280-1287.

3. Barger, P. T. US Patent 5,095,163,1992.

4. Weckhuysen, B. M., Phys. Chem. Chem. Phys. 2003, 5 , 4351-4360.

5. Yilmaz, B.; Müller, U., Top. Catal. 2009, 52, 888-895.

6. Takahashi, H.; Nishi, M.; Tagawa, Y.; Kai, T., Micropor. Mater. 1995, 3, 467-471.

7. Kresge, C. T.; Leonowicz, M. E.; Roth, W. J.; Vartuli, J. C.; Beck, J. S., Nature 1992, 3559, 710-712.

8. Inagaki, S.; Koiwai, A.; Suzuki, N.; Fukushima, Y.; Kuroda, K., Bull. Chem. Soc. Jpn. 1996, 69, 1449-1457.

9. Zhao, D.; Feng, J.; Huo, Q.; Melosh, N.; Fredrickson, G. H.; Chmelka, B. F.; Stucky, G. D., Science 1998, 279, 548-552.

10. (a) Gianotti, E.; Diaz, U.; Velty, A.; Corma, A., Catal. Sci. Technol. 2013, 3, 2677-2688; (b) Vinu, A.; Ariga, K.; Saravanamurugan, S.; Hartmann, M.; Murugesan, V., Micro. Meso. Mater. 2004, 76, 91-98.

11. O'Sullivan, P.; Forni, L.; Hodnett, B. K., Ind. Eng. Chem. Res. 2001, 40, 1471-1475.

12. (a) Gueudré, L.; Milina, M.; Mitchell, S.; Pérez-Ramírez, J., Adv. Funct. Mater. 2014, 24, 209-219; (b) Parlett, C., M, A;; Wilson, K.; Lee, A., Chem. Soc. Rev. 2013, 42, 3876-3893; (c) Li, K.;
Valla, J.; Garcia-Martinez, J., ChemCatChem 2014, 6, 46-66; (d) Takahashi, R.; Sato, S.; Sodesawa, T.; Arai, K.; Yabuki, M.; Journal of Catalysis 2005, 229, 24-29.

13. Potter, M. E.; Paterson, A. J.; Mishara, B.; Kelly, S. D.; Bare, S. R.; Corà, F.; Levy, A. B.; Raja, R., J. Am. Chem. Soc. 2015, DOI: 10.1021/jacs.5b03734.

14. Newland, S. H.; Raja, R.; Levy, A. B., US Patent 62/092,471 filed Dec 2014.

15. Hayashi, S.; Hayamizu, K., Bull. Chem. Soc. Jpn 1991, 64 (2), 685-687.

16. Fung, B. M.; Khitrin, A. K.; Ermolaev, K., J. Magn Reson. 2000, $142(1), 97-101$.

17. Szeverenyi, N. M.; Sullivan, M. J.; Maciel, G. E., J. Magn. Reson 1982, 47, 462-475.

18. van Beek, J. D., Magn. Reson. 2007, 187, 19-26.

19. (a) Sun, Q.; Wang, N.; Xi, D.; Yang, M.; Yu, J., Chem. Commun. 2014, 50, 6502-6505; (b) Choi, M.; Cho, H. S.; Srivastava, R.; Venkatesan, C.; Choi, D.-H.; Ryoo, R., Nature Materials 2006, 5, 718-723; (c) Seo, Y.; Lee, S.; Jo, C.; Ryoo, R., J. Am. Chem. Soc. 2013, 135, 8806-8809.

20. Choi, M.; Srivastava, R.; Ryoo, R., Chem. Commun. 2006, 4380-4382.

21. Potter, M. E.; Cholerton, M. E.; Kezina, J.; Bounds, R.; Carravetta, M.; Manzoli, M.; Gianotti, E.; Lefenfeld, M.; Raja, R., ACS Catal. 2014, 4, 4161-4169.

22. (a) Marthala, V. R. R.; Jiang, Y.; Huang, J.; Wang, W.; Gläser, R.; Hunger, M., J. Am. Chem. Soc. 2006, 128, 14812-14813; (b) Fernández, A. B.; Boronat, M.; Blasco, T.; Corma, A., Angew. Chem. Int. Ed. 2005, 2005, 2370-2373; (c) Heitmann, G. P.; Dahlhoff, G.; Holderich, W. F., Journal of Catalysis 1999, 186, 12-19; (d) Icihashi, H.; Kitamura, M., Catalysis Today 2002, 73, 23-28.

23. Icihashi, H.; Sato, H., Appl. Catal. 2001, 221, 359-366.

24. Kim, J.; Park, W.; Ryoo, R., ACS Catal. 2011, 1, 337-341. 


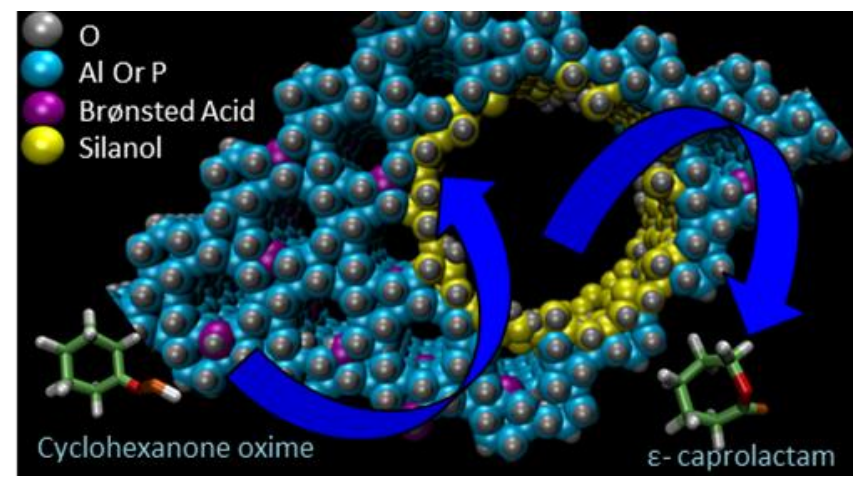




\section{SUPPORTING INFORMATION}

\section{Expanding Beyond the Micropore: Active-Site Engineering in}

Hierarchical Architectures for Beckmann Rearrangement

Stephanie H. Newland, ${ }^{ \pm}$Wharton Sinkler,${ }^{\S}$ Thomas Mezza, ${ }^{\S}$ Simon R. Bare,${ }^{\S}$ Marina Carravetta ${ }^{ \pm}$, Ibraheem M. Haies, ${ }^{ \pm}$Alan Levy, ${ }^{\phi}$ Scott Keenan, ${ }^{\phi}$ Robert Raja ${ }^{ \pm *}$

${ }^{ \pm}$School of Chemistry, University of Southampton, Southampton S017 1BJ, UK §UOP, a Honeywell company, Des Plaines, IL 60017, USA

$\phi$ Honeywell Int., 101 Columbia Road, Morristown, NJ 0762, USA

*R.Raja@soton.ac.uk

\section{Experimental and Additional Characterization}

\section{Contents}

Active sites, Substitution Mechanisms and Structures of SAPOs

Page S2

Synthesis of Catalysts

Page S4

Powder XRD and cell parameter refinement values

Page S9

Characterization of Catalysts Physical Properties- Scanning Electron Microscopy, Surface Area Analysis and Metal Content

Vapour Phase Beckmann Rearrangement of Cyclohexanone Oxime

Page S22

Characterization of Catalysts Acidic Properties - NMR, FT-IR

Page S26

Vapour Phase Beckmann Rearrangement of Cyclooctanone Oxime 


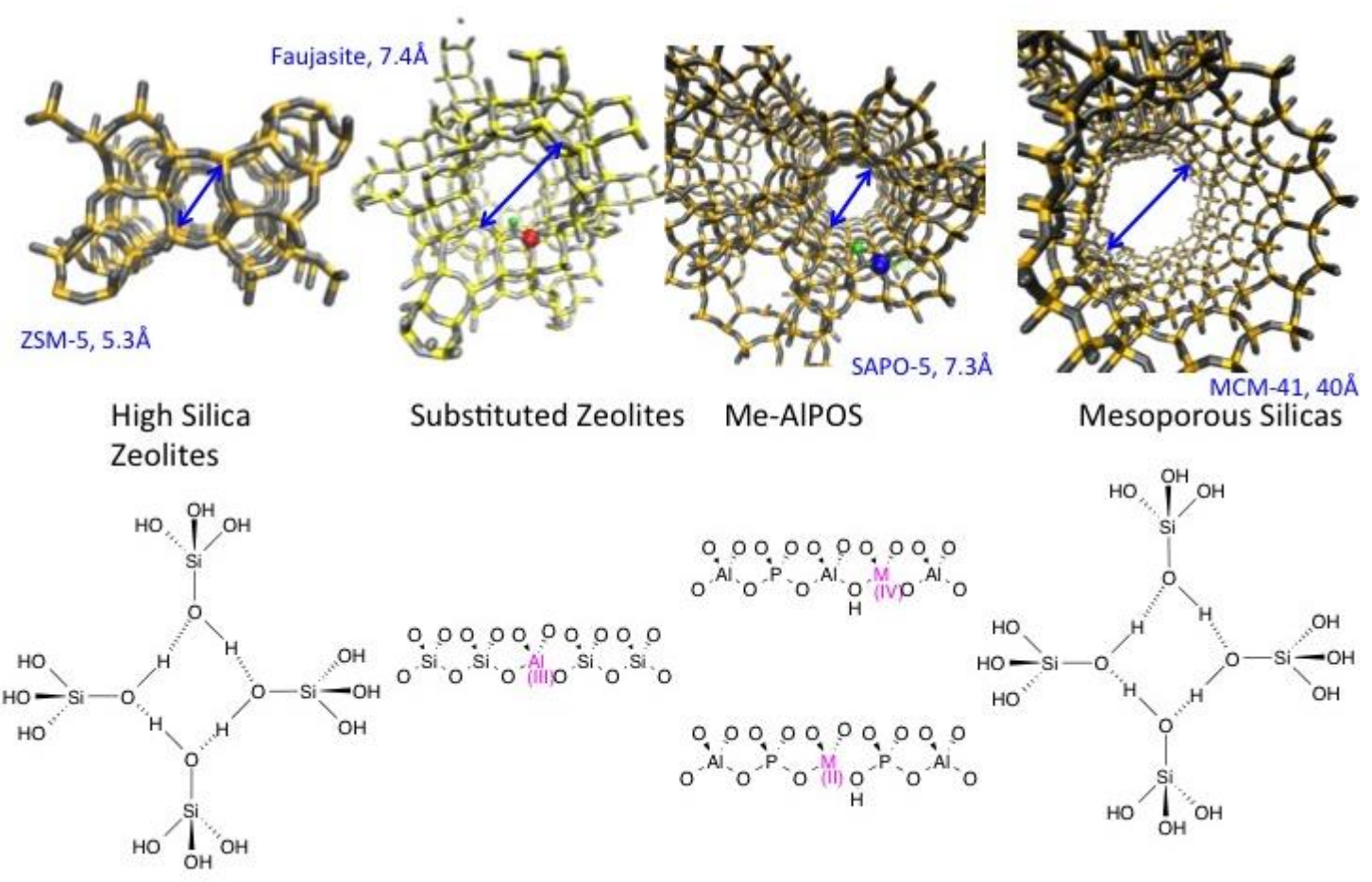

Figure S1: Origin of isolated active sites in zeotypes.

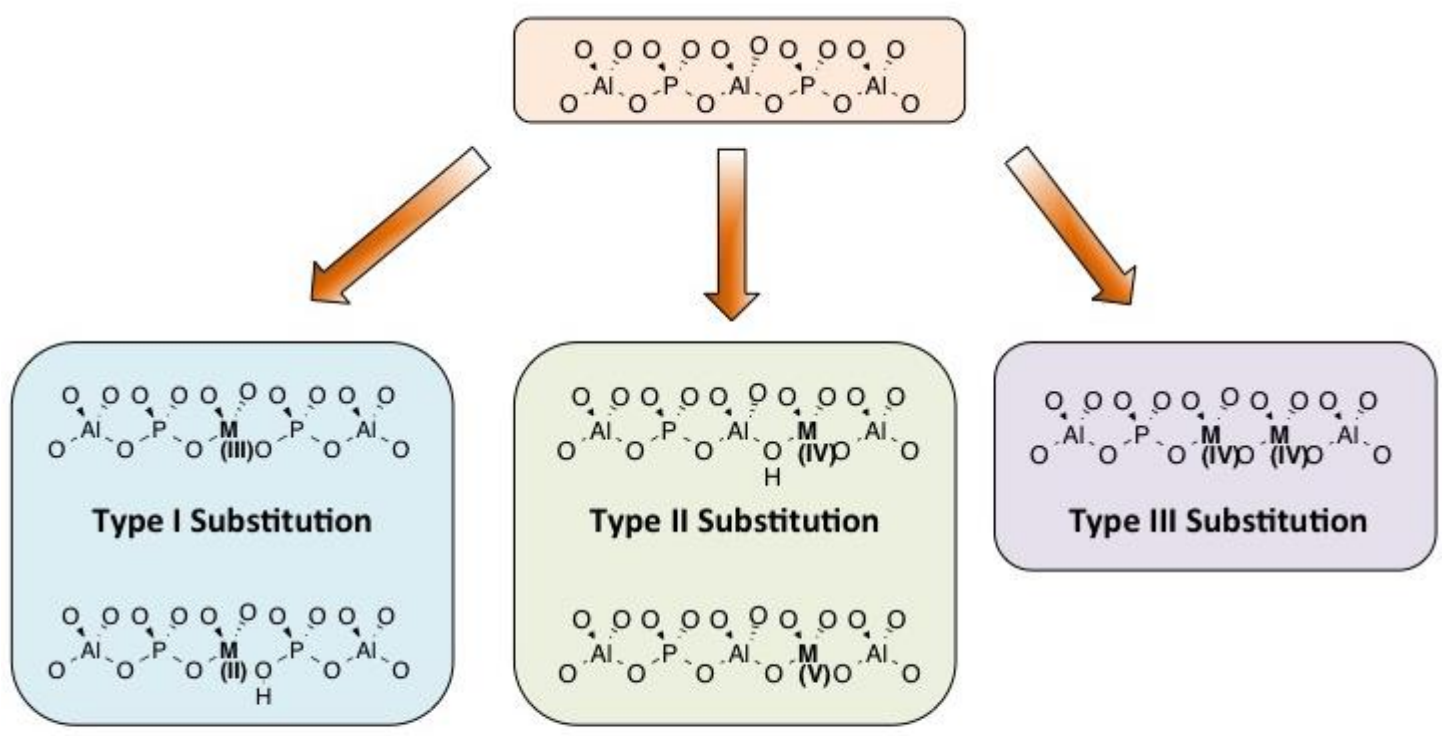

Figure S2: Different substitution mechanisms in AlPO frameworks and the origin of Brønsted acid sites arising from framework substitution of Si(IV) atoms for $\mathrm{Al}(\mathrm{III})$ and $\mathrm{P}(\mathrm{V})$. 

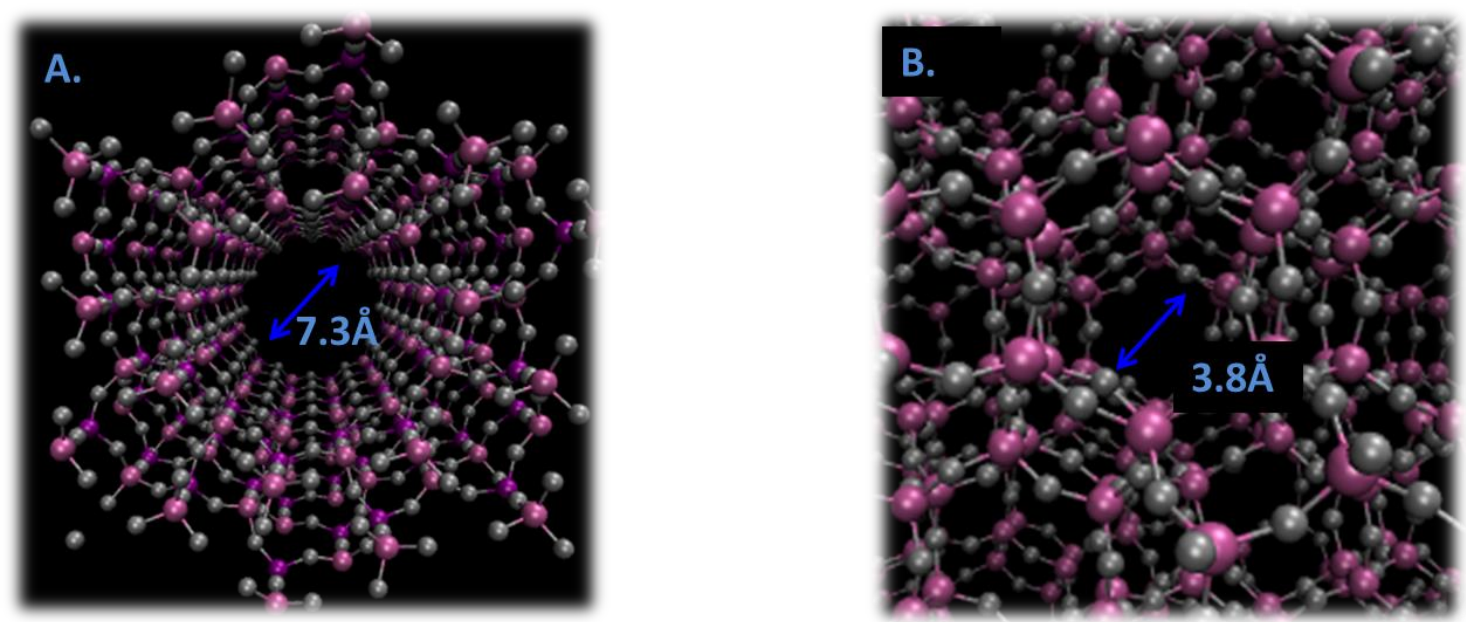

Figure S3: Graphical representation of the microporous SAPO-5 (A) and SAPO34 (B) cages with pore dimensions.

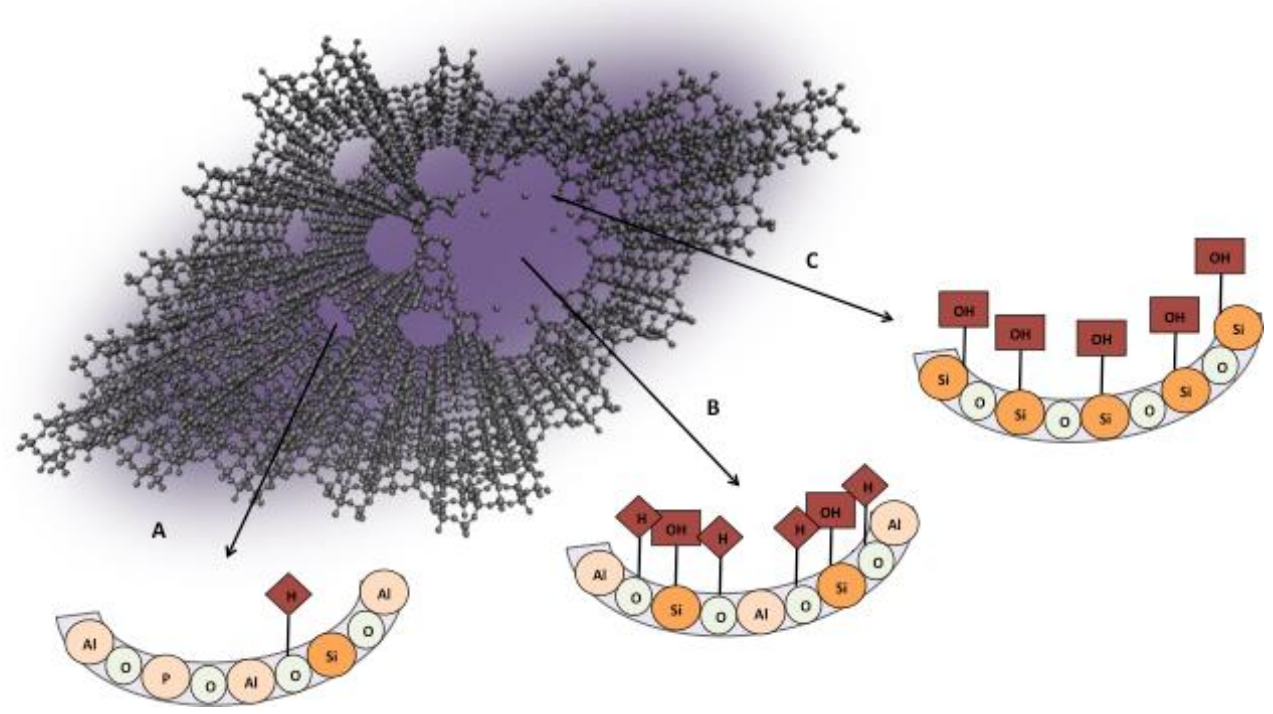

Figure S4: Potential acid sites present in the hierarchical SAPO framework. It is believed that the micropores will contain acid-sites originating from type II substitution (Si(IV) replacing a framework P(V)), (A) and/or type III substitution (2Si(IV) replacing an adjacent $\mathrm{Al}(\mathrm{III})$ and $\mathrm{P}(\mathrm{V})$ - see Figure S1.2). Within the mesopores, acid sites could additionally originate from the calcination of the embedded surfactant to reveal silanols (B), which may saturate the lining of the mesopores (C). 


\section{Synthesis of Catalysts}

Table S1: Inductively Coupled Plasma (ICP) chemical analysis of SAPO Catalysts

\begin{tabular}{|l|l|l|l|}
\hline Catalyst $^{\mathbf{a}}$ & $\mathbf{A l} / \mathbf{w t} \%$ & $\mathbf{P} / \mathbf{w t} \%$ & $\mathbf{S i} / \mathbf{w t} \%$ \\
\hline SAPO-5 & 22.61 & 19.98 & 1.49 \\
\hline SAPO-34 & 22.78 & 17.14 & 3.24 \\
\hline HP SAPO-5 & 20.60 & 20.20 & 6.63 \\
\hline HP SAPO-34 & 24.10 & 18.20 & 5.08 \\
\hline
\end{tabular}

${ }^{\text {aH}} \mathrm{H}-\mathrm{ZSM}-5(\mathrm{Si} / \mathrm{Al}=30)$ was supplied by the UK Catalysis Hub. MCM-41 was synthesised according to published procedures. ${ }^{1}$

\section{Thermal Gravimetric Analysis (TGA)}

The presence of a prominent mass loss at approximately $350^{\circ} \mathrm{C}$ for the hierarchically porous samples indicated that the surfactant (DMOD) was successfully incorporated into the SAPO framework. This peak was absent in the microporous analogues. The TGA showed similar features between the microporous and hierarchically porous equivalents apart from the presence of that peak.

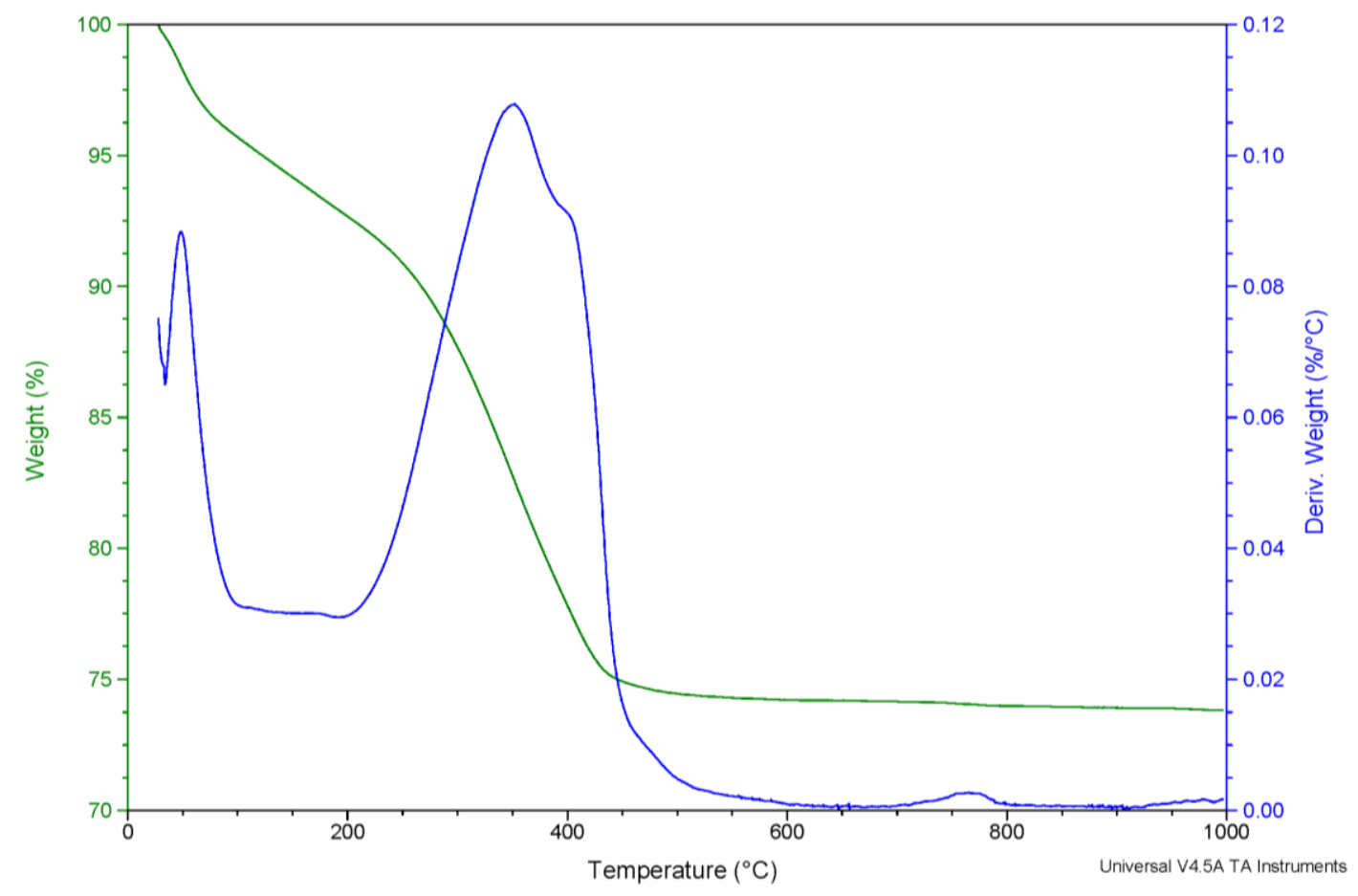

Figure S5: TGA analysis of HP SAPO-5. 


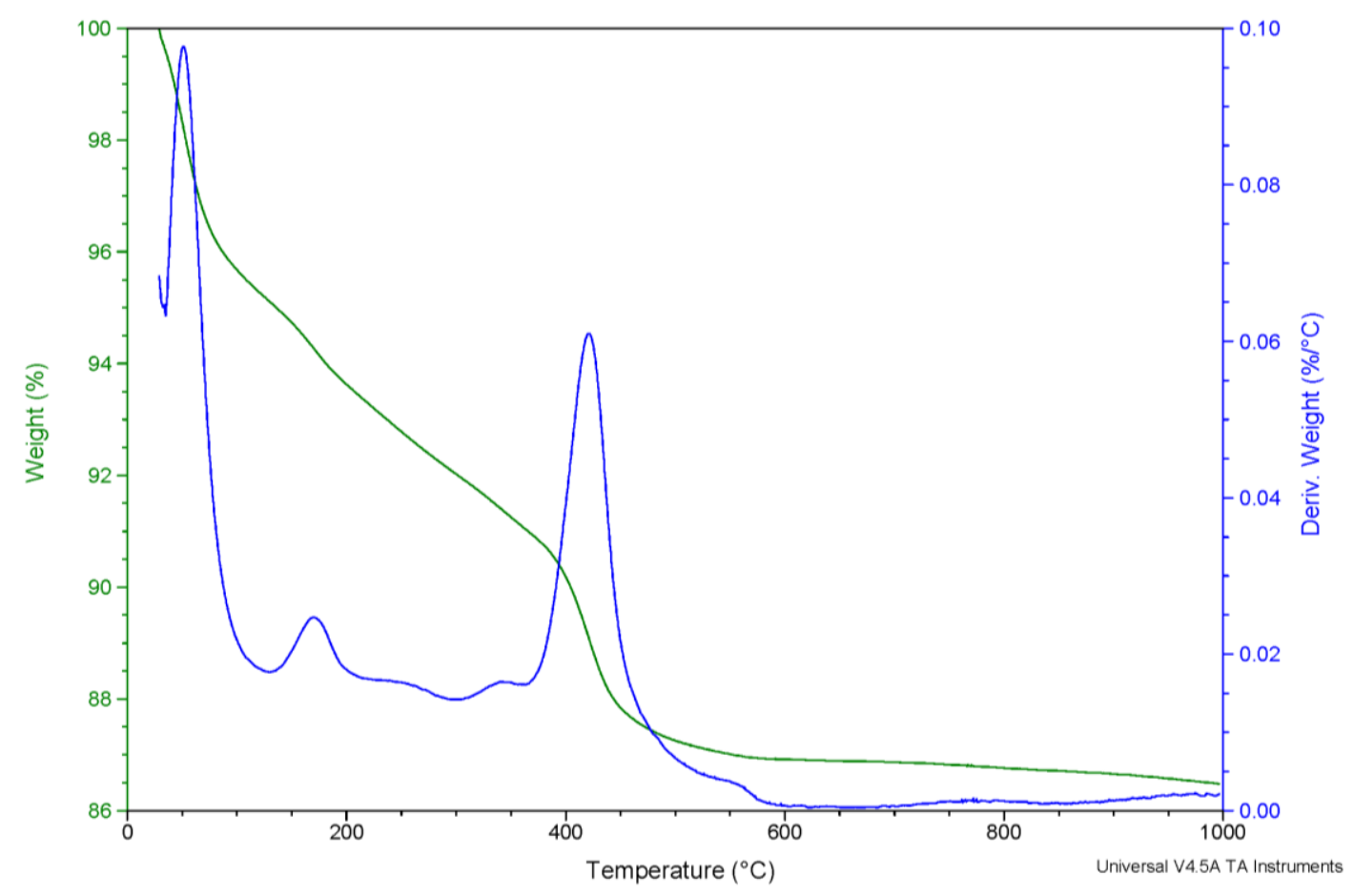

Figure S6: TGA analysis of SAPO-5.

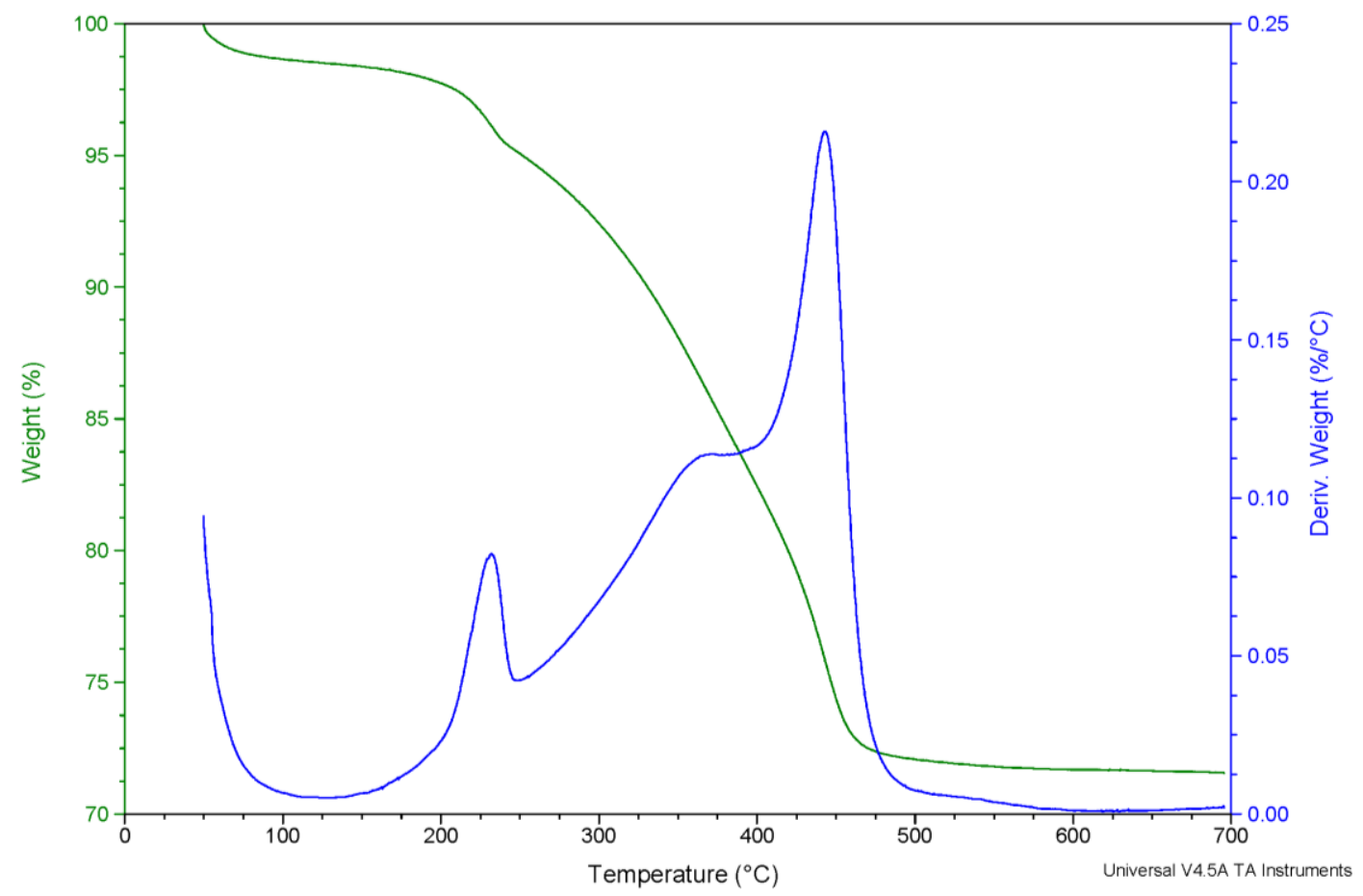

Figure S7: TGA analysis of HP SAPO-34. 


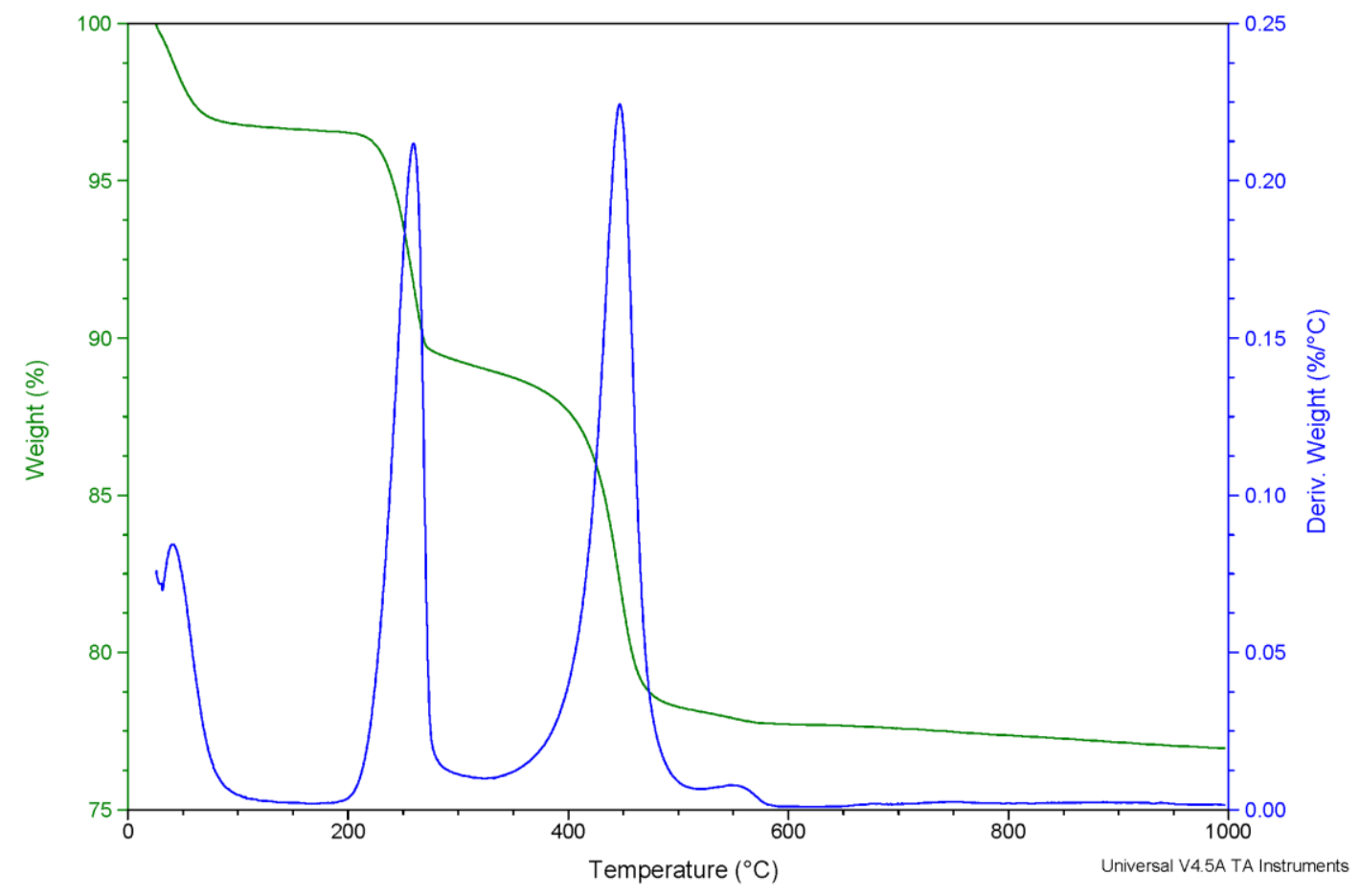

Figure S8: TGA analysis of SAPO-34. 


\section{Structural characterization: Powder XRD and cell parameter refinement}

values

Powder X-ray Diffraction (XRD) revealed that the hierarchical SAPO-5 had the expected AFI framework with no detectable impurities (Figure S9). A peak at low-angle, which is present in the hierarchical material and absent in the microporous, is indicative of the presence of mesopores.

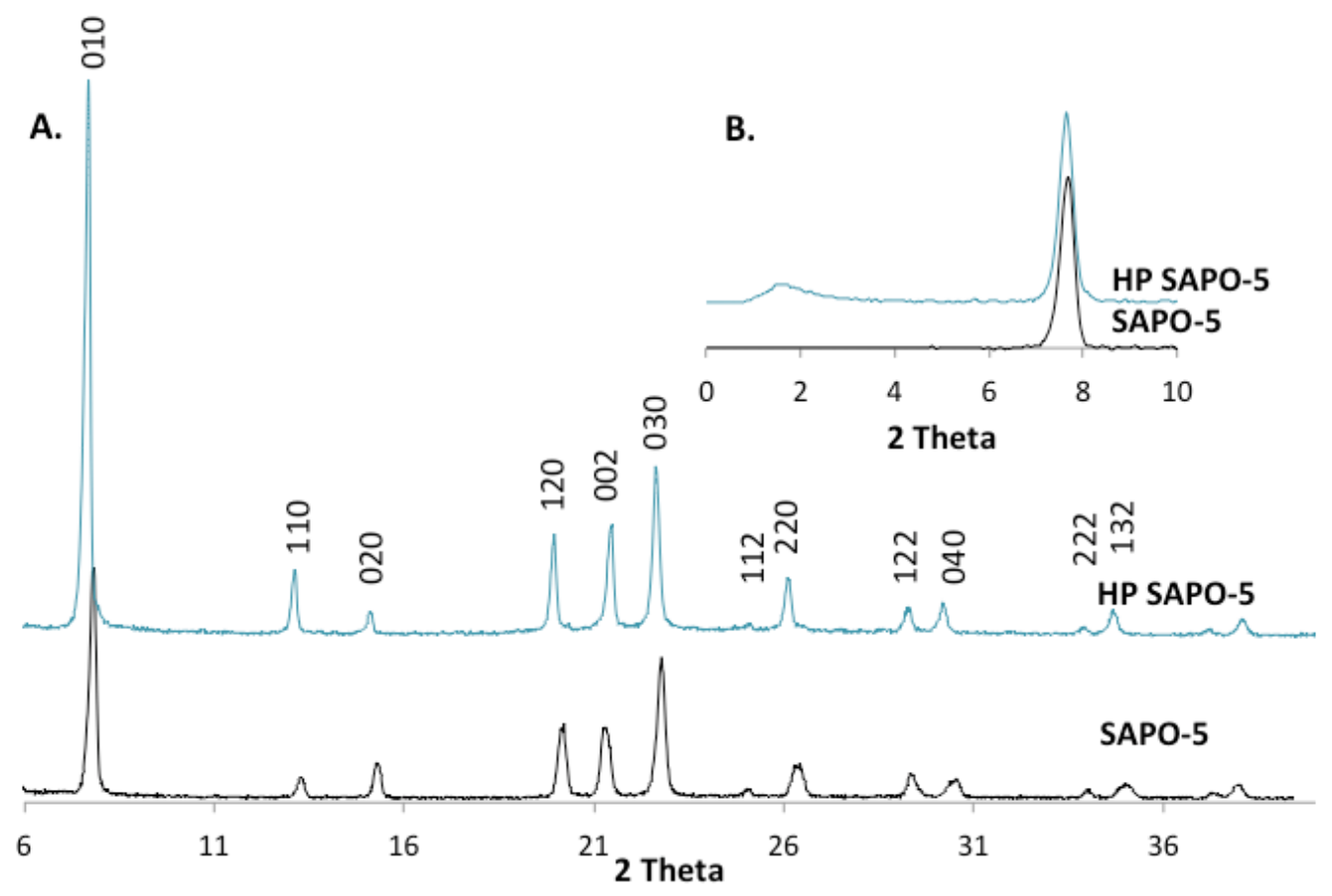

Figure S9: Powder XRD of microporous and hierarchically porous (HP) SAPO-5 (A). Inset (B) is low angle powder XRD of the samples. 


\section{Retiveld Refinements}

Table S2: CelRef refinement values for calcined SAPO-5. Initial values : (Refinement keys on 2nd line)

\begin{tabular}{|c|c|c|c|c|c|c|c|c|}
\hline Zero & Lambda & a & b & c & alpha & beta & gamma & Vol. \\
\hline 0 & 1.5406 & 13.5124 & 13.5124 & 8.3871 & 90 & 90 & 120 & 1326.2 \\
\hline 0 & 0 & 1 & 0 & 1 & 0 & 0 & 0 & \\
\hline
\end{tabular}

Final values : (Standard errors on 2 nd line)

\begin{tabular}{|c|c|c|c|c|c|c|c|}
\hline \multirow{2}{*}{ Zero } & & Lambda & $a$ & b & c & \multicolumn{2}{|l|}{ alpha } \\
\hline & 0 & 1.5406 & 13.5126 & 13.5126 & 8.3871 & 90 & 90 \\
\hline & 0 & 0 & 0.0908 & 0 & 0.0074 & 0 & 0 \\
\hline \multirow[t]{15}{*}{$\mathrm{H}$} & & $\mathrm{K}$ & $\mathrm{L}$ & $2 \mathrm{~T}$ (Obs) & 2T-Zero & 2Th(Cal) & Dif \\
\hline & 0 & 1 & 0 & 7.818 & 7.818 & 7.5484 & 0.2696 \\
\hline & 1 & 1 & 0 & 13.247 & 13.247 & 13.0933 & 0.1537 \\
\hline & 0 & 2 & 0 & 15.273 & 15.273 & 15.1299 & 0.1431 \\
\hline & 1 & 2 & 0 & 20.215 & 20.215 & 20.0591 & 0.1559 \\
\hline & 0 & 0 & 2 & 21.248 & 21.248 & 21.1691 & 0.0789 \\
\hline & 0 & 3 & 0 & 22.788 & 22.788 & 22.7786 & 0.0094 \\
\hline & 1 & 1 & 2 & 24.996 & 24.996 & 24.971 & 0.025 \\
\hline & 2 & 2 & 0 & 26.292 & 26.292 & 26.3615 & -0.0695 \\
\hline & 1 & 2 & 2 & 29.351 & 29.351 & 29.3247 & 0.0263 \\
\hline & 0 & 4 & 0 & 30.364 & 30.364 & 30.5319 & -0.1679 \\
\hline & 2 & 2 & 2 & 33.99 & 33.99 & 34.0521 & -0.0621 \\
\hline & 1 & 3 & 2 & 34.861 & 34.861 & 34.9289 & -0.0679 \\
\hline & 0 & 4 & 2 & 37.434 & 37.434 & 37.4518 & -0.0178 \\
\hline & 1 & 2 & 3 & 38.0416 & 38.0416 & 38.0469 & -0.0053 \\
\hline
\end{tabular}

Table S3: CelRef refinement values for calcined HP SAPO-5.

\begin{tabular}{|c|c|c|c|c|c|c|c|}
\hline \multicolumn{8}{|c|}{ Initial values : (Refinement keys on 2 nd line) } \\
\hline Zero & & Lambda & a & b & c & alpha & beta \\
\hline & 0 & 1.5406 & 13.6494 & 13.6494 & 8.3233 & 90 & 90 \\
\hline & 0 & 0 & 1 & 0 & 1 & 0 & 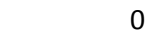 \\
\hline \multicolumn{8}{|c|}{ Final values $\quad:$ (Standard errors on 2 nd line) } \\
\hline Zero & & Lambda & a & $\mathrm{b}$ & c & alpha & beta \\
\hline & 0 & 1.5406 & 13.651 & 13.651 & 8.3234 & 90 & 90 \\
\hline & 0 & 0 & 0.0672 & 0 & 0.0075 & 0 & 0 \\
\hline $\mathrm{H}$ & & $\mathrm{K}$ & $\mathrm{L}$ & $2 \mathrm{~T}$ (Obs) & 2T-Zero & 2Th(Cal) & Dif \\
\hline & 1 & 1 & 0 & 13.113 & 13.113 & 12.96 & 0.153 \\
\hline & 0 & 2 & 0 & 15.118 & 15.118 & 14.9756 & 0.1424 \\
\hline & 1 & 2 & 0 & 19.954 & 19.954 & 19.8538 & 0.1002 \\
\hline & 0 & 0 & 2 & 21.469 & 21.469 & 21.333 & 0.136 \\
\hline & 0 & 1 & 2 & 22.628 & 22.628 & 22.6327 & -0.0047 \\
\hline & 1 & 1 & 2 & 25.057 & 25.057 & 25.0405 & 0.0165 \\
\hline & 2 & 2 & 0 & 26.104 & 26.104 & 26.0896 & 0.0144 \\
\hline & 1 & 3 & 1 & 29.246 & 29.246 & 29.2511 & -0.0051 \\
\hline & 0 & 4 & 0 & 30.182 & 30.182 & 30.215 & -0.033 \\
\hline & 2 & 2 & 2 & 33.836 & 33.836 & 33.9434 & -0.1074 \\
\hline & 1 & 4 & 0 & 34.661 & 34.661 & 34.7459 & -0.0849 \\
\hline & 0 & 4 & 2 & 37.179 & 37.179 & 37.2857 & -0.1067 \\
\hline & 0 & 5 & 0 & 38.0254 & 38.0254 & 38.0267 & -0.0013 \\
\hline
\end{tabular}


Table S4: CelRef refinement values for calcined SAPO-34.

Initial values : (Refinement keys on 2 nd line)

\begin{tabular}{|c|c|c|c|c|c|c|c|c|}
\hline Zero & Lambda & a & $\mathrm{b}$ & c & alpha & beta & gamma & Vol. \\
\hline 0 & 1.5406 & 9.395 & 9.395 & 9.395 & 94.23 & 94.23 & 94.23 & 822.1 \\
\hline 0 & 0 & 1 & 0 & 0 & 1 & 0 & 0 & \\
\hline \multicolumn{9}{|c|}{ Final values : (Standard errors on 2 nd line) } \\
\hline Zero & Lambda & $\mathrm{a}$ & $\mathrm{b}$ & $\mathrm{c}$ & alpha & beta & gamma & Vol. \\
\hline 0 & 1.5406 & 9.3943 & 9.3943 & 9.3943 & 94.23 & 94.23 & 94.23 & 822 \\
\hline 0 & 0 & 0.0405 & 0 & 0 & 0.248 & 0 & 0 & \\
\hline $\mathrm{H}$ & K & $L$ & $2 \mathrm{~T}(\mathrm{Obs})$ & 2T-Zero & 2Th(Cal) & Dif & & \\
\hline 0 & 0 & -1 & 9.459 & 9.459 & 9.4625 & -0.0035 & & \\
\hline 0 & 1 & -1 & 12.846 & 12.846 & 12.8504 & -0.0044 & & \\
\hline 0 & 1 & 1 & 13.826 & 13.826 & 13.9231 & -0.0971 & & \\
\hline 0 & 1 & 1 & 13.846 & 13.846 & 13.9231 & -0.0771 & & \\
\hline 1 & 1 & -1 & 15.988 & 15.988 & 15.9822 & 0.0058 & & \\
\hline 1 & 1 & 1 & 17.659 & 17.659 & 17.6968 & -0.0378 & & \\
\hline 0 & 0 & -2 & 18.974 & 18.974 & 18.9904 & -0.0164 & & \\
\hline 0 & 1 & -2 & 20.578 & 20.578 & 20.5606 & 0.0174 & & \\
\hline 0 & 1 & 2 & 21.982 & 21.982 & 21.9313 & 0.0507 & & \\
\hline 1 & 1 & -2 & 22.383 & 22.383 & 22.3525 & 0.0305 & & \\
\hline 1 & 2 & -1 & 23.007 & 23.007 & 22.9967 & 0.0103 & & \\
\hline 1 & 1 & 2 & 24.878 & 24.878 & 24.8346 & 0.0434 & & \\
\hline 0 & 2 & -2 & 25.881 & 25.881 & 25.8662 & 0.0148 & & \\
\hline 1 & 2 & -2 & 27.619 & 27.619 & 27.5993 & 0.0197 & & \\
\hline 0 & 2 & 2 & 28.065 & 28.065 & 28.0573 & 0.0077 & & \\
\hline 0 & 3 & -1 & 29.558 & 29.558 & 29.4908 & 0.0672 & & \\
\hline 1 & 1 & -3 & 30.583 & 30.583 & 30.5526 & 0.0304 & & \\
\hline 1 & 3 & -1 & 31.251 & 31.251 & 31.516 & -0.265 & & \\
\hline 2 & 2 & -2 & 32.321 & 32.321 & 32.2864 & 0.0346 & & \\
\hline 1 & 1 & 3 & 33.368 & 33.368 & 33.3679 & 0.0001 & & \\
\hline 1 & 2 & -3 & 34.438 & 34.438 & 34.4442 & -0.0062 & & \\
\hline 0 & 2 & 3 & 35.953 & 35.953 & 35.8933 & 0.0597 & & \\
\hline 0 & 0 & 4 & 38.583 & 38.583 & 38.529 & 0.054 & & \\
\hline 1 & 1 & -4 & 39.6743 & 39.6743 & 39.6236 & 0.0507 & & \\
\hline
\end{tabular}

Table S5: CelRef refinement values for calcined HP SAPO-34.

Initial values : (Refinement keys on 2 nd line)

\begin{tabular}{|c|c|c|c|c|c|c|c|c|c|}
\hline \multirow[t]{3}{*}{ Zero } & \multicolumn{2}{|r|}{ Lambda } & \multicolumn{2}{|r|}{$b$} & $c$ & alpha & beta & gamma & \multirow[t]{2}{*}{ Vol. } \\
\hline & 0 & 1.5406 & 9.3684 & 9.3684 & 9.3684 & 94.26 & 94.26 & 94.26 & \\
\hline & 0 & 0 & 1 & 0 & 0 & 1 & 0 & 0 & \\
\hline \multicolumn{10}{|c|}{ Final values : (Standard errors on 2 nd line) } \\
\hline Zero & & Lambda & a & $\mathrm{b}$ & c & alpha & beta & gamma & Vol. \\
\hline & 0 & 1.5406 & 9.368 & 9.368 & 9.368 & 94.25 & 94.25 & 94.25 & 815 \\
\hline & 0 & 0 & 0.0563 & 0 & 0 & 0.494 & 0 & 0 & \\
\hline $\mathrm{H}$ & & $\mathrm{K}$ & $\mathrm{L}$ & $2 \mathrm{~T}(\mathrm{Obs})$ & 2T-Zero & 2Th(Cal) & Dif & & \\
\hline & 0 & 0 & -1 & 9.615 & 9.615 & 9.4898 & 0.1252 & & \\
\hline & 0 & 1 & -1 & 12.979 & 12.979 & 12.8845 & 0.0945 & & \\
\hline & 1 & 1 & -1 & 16.143 & 16.143 & 16.026 & 0.117 & & \\
\hline & 1 & 1 & 1 & 17.837 & 17.837 & 17.7548 & 0.0822 & & \\
\hline & 0 & 0 & -2 & 19.085 & 19.085 & 19.0454 & 0.0396 & & \\
\hline & 0 & 1 & -2 & 20.734 & 20.734 & 20.6165 & 0.1175 & & \\
\hline & 1 & 2 & -1 & 23.207 & 23.207 & 23.062 & 0.145 & & \\
\hline & 1 & 1 & 2 & 25.057 & 25.057 & 24.9153 & 0.1417 & & \\
\hline & 0 & 2 & -2 & 26.015 & 26.015 & 25.9355 & 0.0795 & & \\
\hline & 1 & 2 & -2 & 27.686 & 27.686 & 27.6743 & 0.0117 & & \\
\hline & 0 & 2 & 2 & 27.953 & 27.953 & 28.1454 & -0.1924 & & \\
\hline & 1 & 2 & 2 & 30.761 & 30.761 & 30.7617 & -0.0007 & & \\
\hline & 1 & 2 & 2 & 30.873 & 30.873 & 30.7617 & 0.1113 & & \\
\hline & 2 & 2 & -2 & 32.209 & 32.209 & 32.3766 & -0.1676 & & \\
\hline & 1 & 2 & -3 & 34.349 & 34.349 & 34.5379 & -0.1889 & & \\
\hline & 2 & 2 & 2 & 35.908 & 35.908 & 35.9548 & -0.0468 & & \\
\hline
\end{tabular}




\section{Structural (HRTEM), textural (BET, adsorption), Morphological (SEM) and elemental (EDXS) characterization of Catalysts}

\section{$\mathrm{N}_{2}$ adsorption/ desorption isotherm}

Nitrogen adsorption-desorption experiments were performed using a Gemini 2375 BET Apparatus with nitrogen as the adsorption gas at $77 \mathrm{~K}$.

The $\mathrm{N}_{2}$ adsorption desorption isotherm of hierarchically porous HP SAPO-5 is typical of a type IV isotherm with a hysteresis which indicates the presence of mesopores (Figure S10). The BJH curve indicates the inclusion of mesopores between $20-60 \AA$ being present.

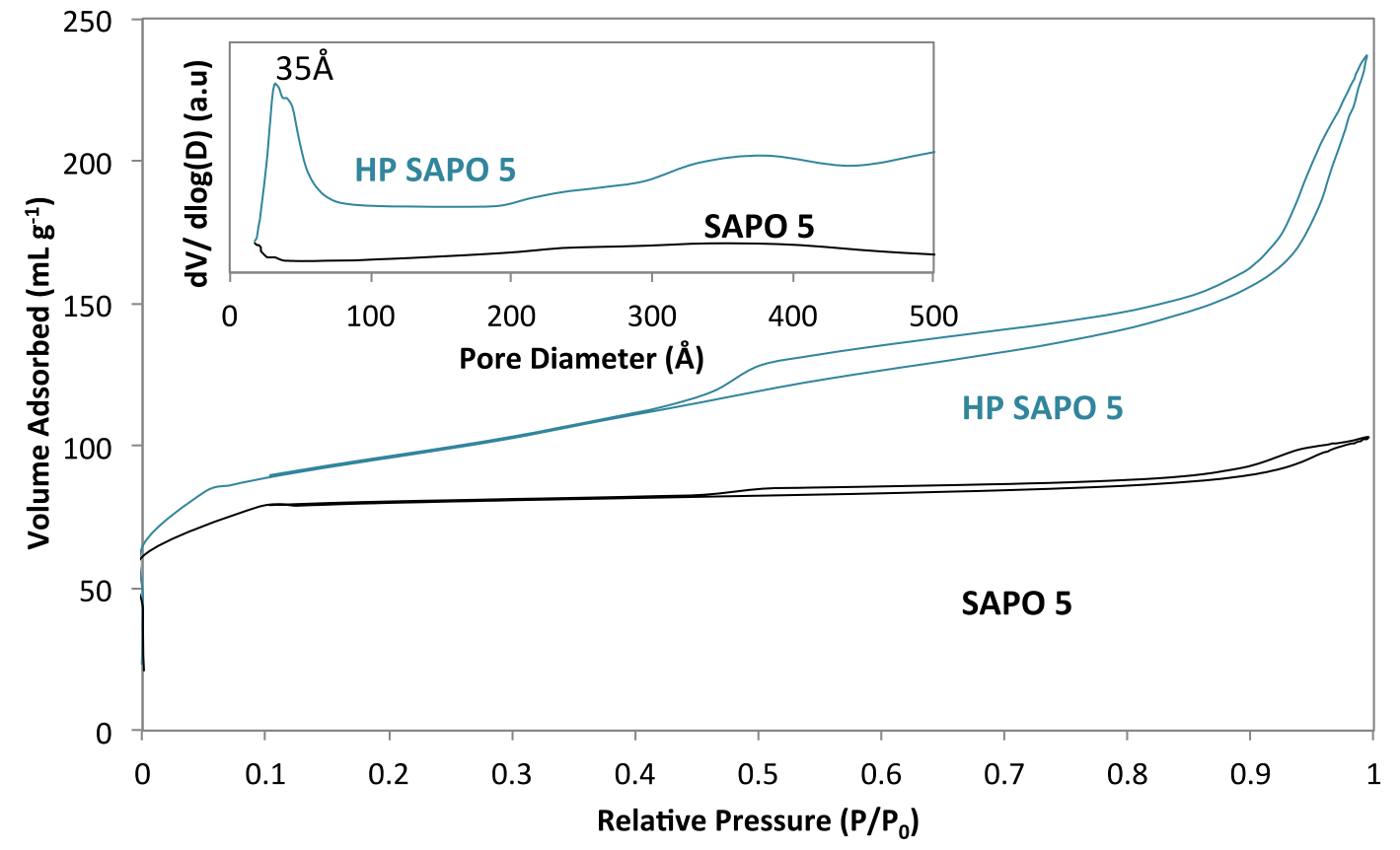

Figure S10: $\mathrm{N}_{2}$ adsorption/ desorption isotherm of microporous SAPO-5 and hierarchical SAPO 5 with BJH adsorption pore distribution inset.

Table S6: BET textual properties

\begin{tabular}{l|llll}
\hline Sample & S BET $\left(\mathrm{m}^{2} \mathrm{~g}^{-1}\right)$ & $\mathrm{V}_{\text {micro }}\left(\mathrm{cm}^{3} \mathrm{~g}^{-1}\right)$ & $\mathrm{V}_{\text {meso }}\left(\mathrm{cm}^{3} \mathrm{~g}^{-1}\right)$ & External surface area \\
& & & & \\
\hline SAPO-5 & 253.8 & 0.06 & 0.05 & 26.0 \\
HP SAPO-5 & 315.8 & 0.08 & 0.30 & 121.7 \\
SAPO-34 & 579.5 & 0.27 & 0.06 & 32.0 \\
HP SAPO-34 & 602.6 & 0.24 & 0.22 & 96.8 \\
\hline
\end{tabular}


Scanning Electron Microscopy (SEM) and High-Resolution Transmission Electron Microscopy (HRTEM)

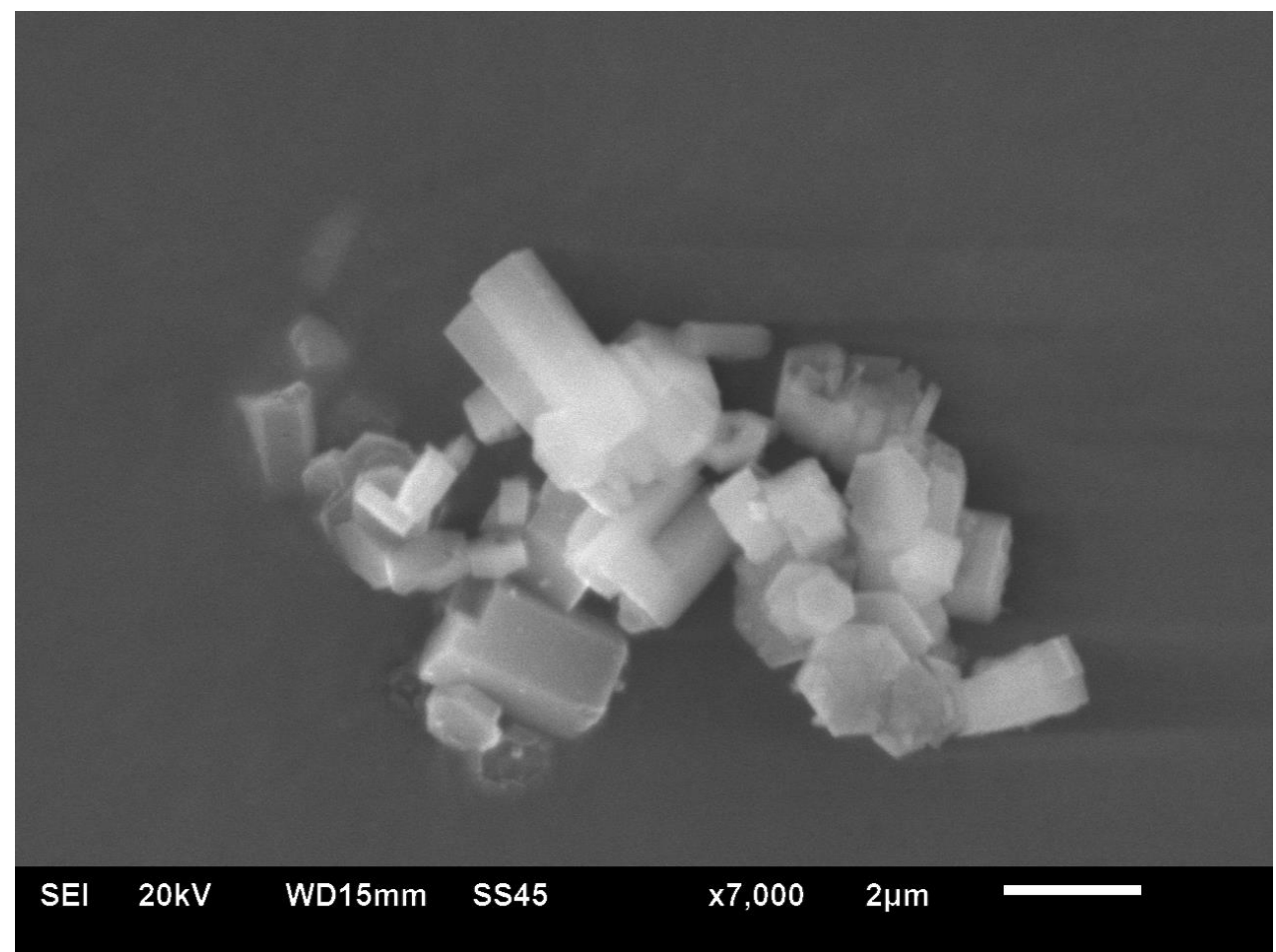

Figure S11: SEM of microporous SAPO-5 revealed elongated hexagonal crystals

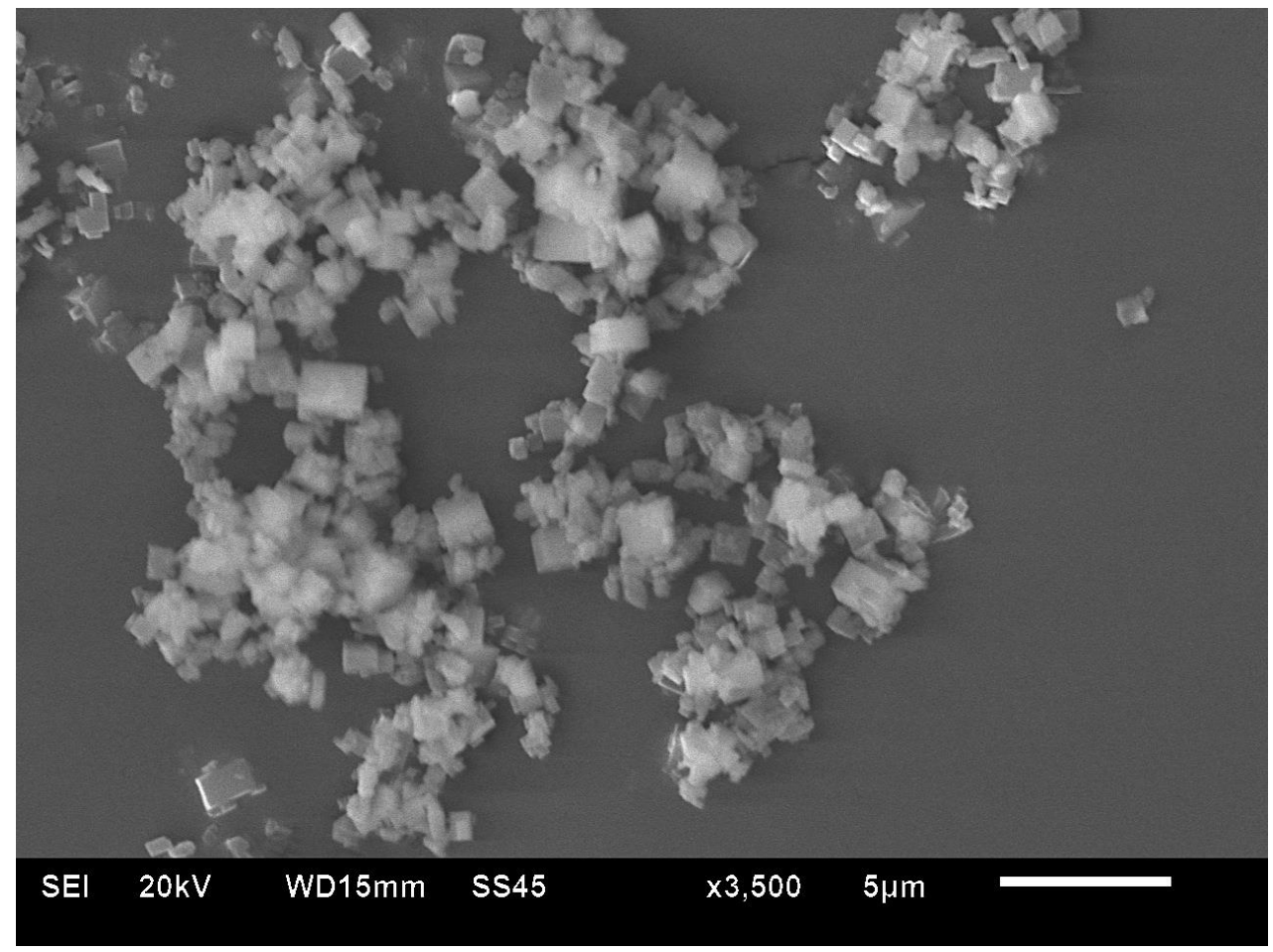

Figure S12: SEM of microporous SAPO-34 revealed cubic crystals. 


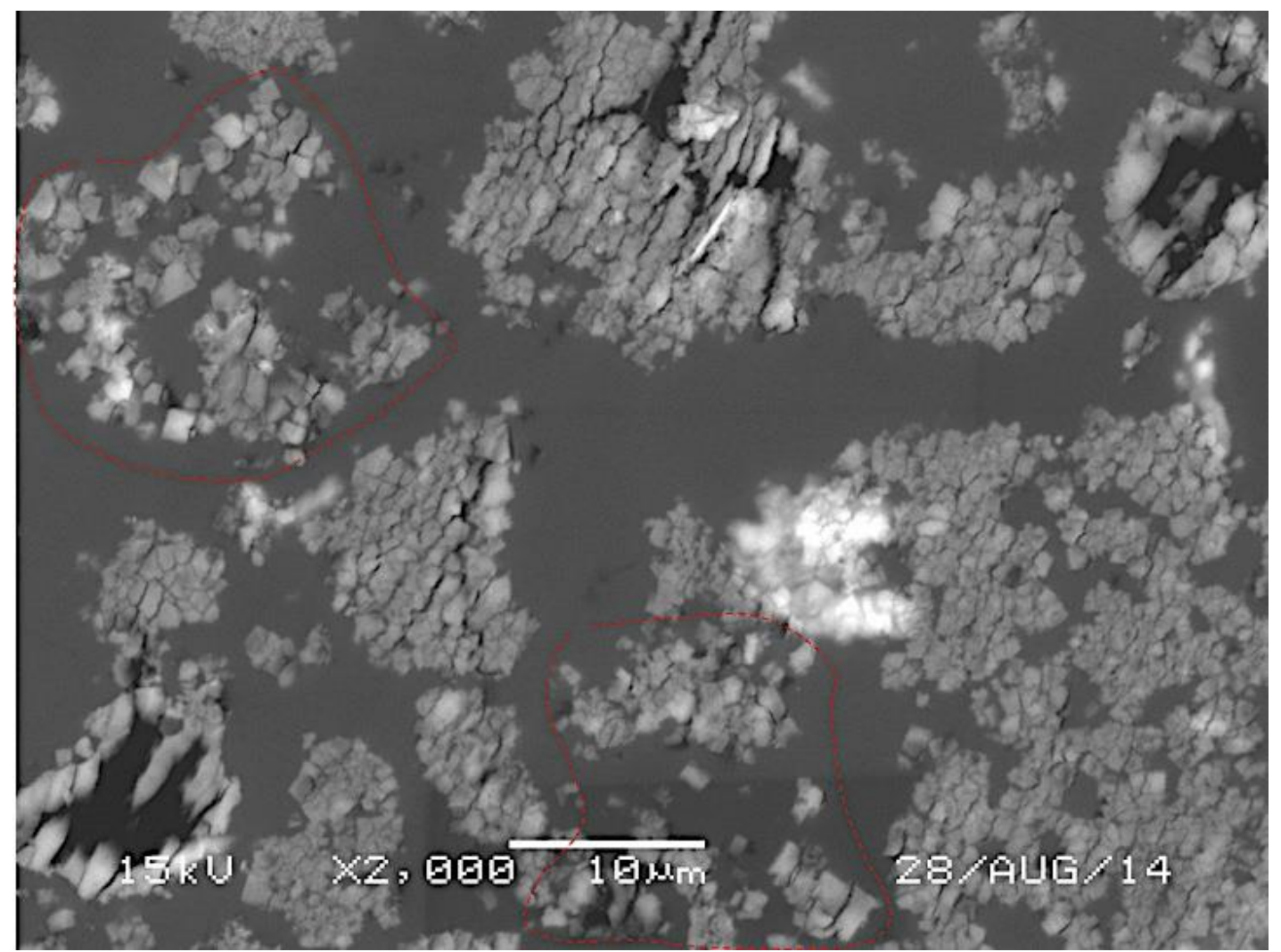

Figures S13: High resolution SEM of HP SAPO-34 shows that the material is composed of well-dispersed crystals (red dotted lines).

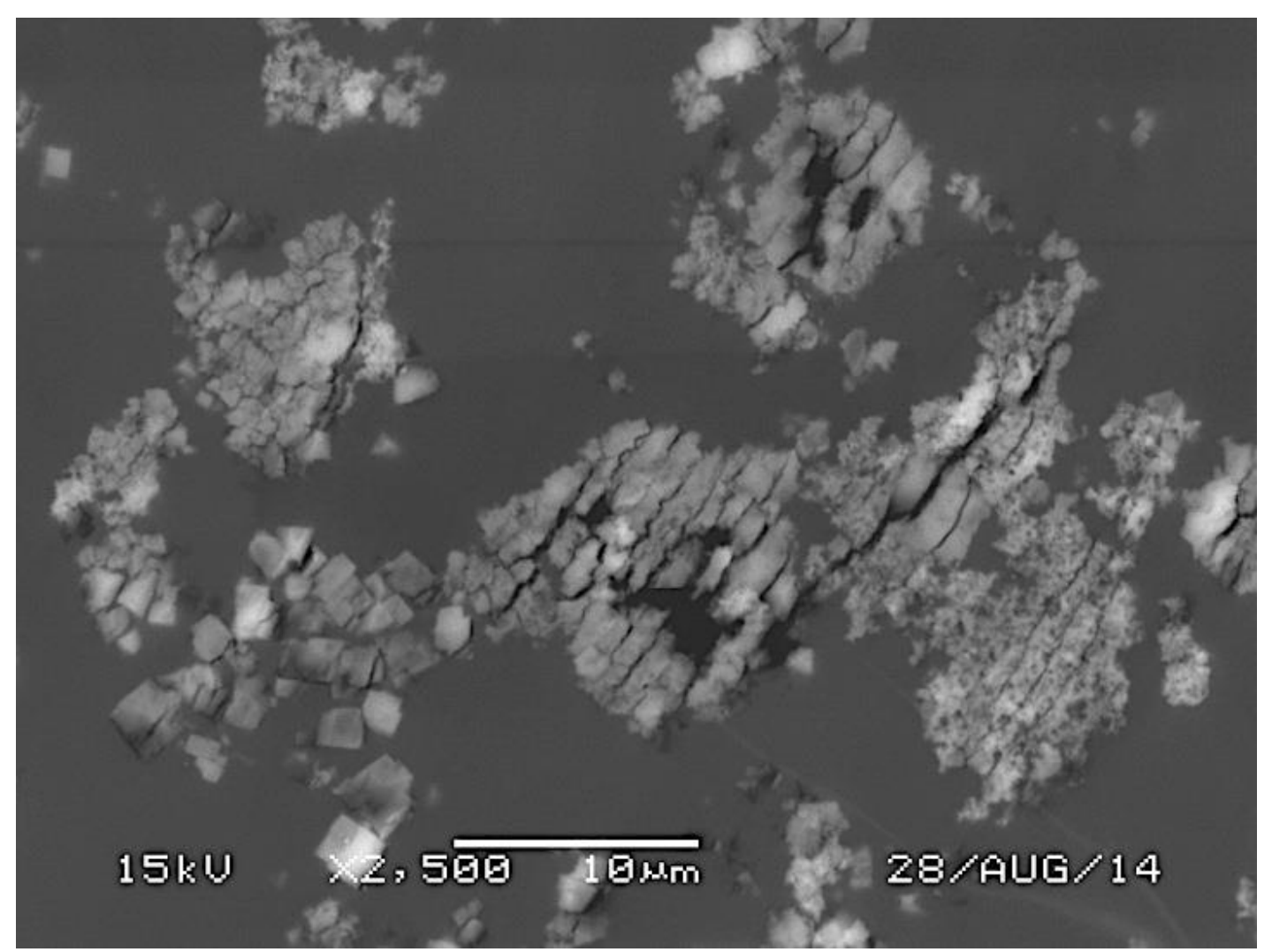

Figure S14: High resolution SEM of HP SAPO-34 detailing evidence of blocky crystals. 


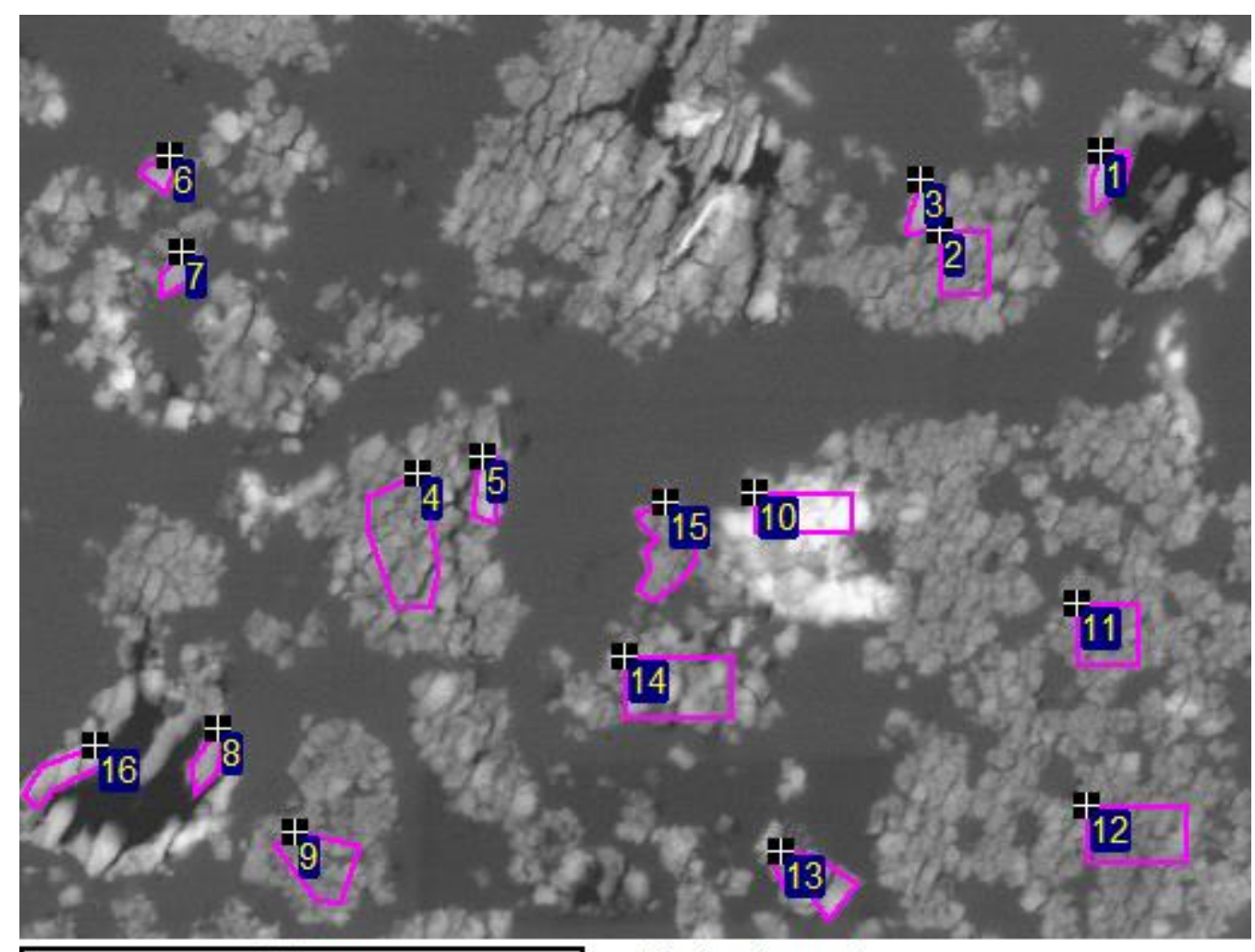

\begin{tabular}{|c|c|c|c|c|c|c|c|c|}
\hline \multicolumn{4}{|c|}{$30 \mu \mathrm{m}$} & \multicolumn{5}{|c|}{ Electron Image 1} \\
\hline Spec. & $\begin{array}{l}\text { wt\% } \\
\text { Al }\end{array}$ & $+/-$ & $\begin{array}{l}\text { wt\% } \\
\text { Si }\end{array}$ & $+/-$ & wt\% P & $+/-$ & $\begin{array}{l}\text { wt\% } \\
0\end{array}$ & $+/-$ \\
\hline 1 & 20.9 & 0.2 & 4.6 & 0.2 & 22.1 & 0.3 & 52.4 & 0.8 \\
\hline 2 & 20.8 & 0.3 & 4.5 & 0.2 & 22.3 & 0.1 & 52.4 & 1.0 \\
\hline 3 & 20.9 & 0.2 & 4.4 & 0.2 & 22.3 & 0.3 & 52.4 & 0.8 \\
\hline 4 & 21.2 & 0.3 & 4.3 & 0.2 & 22.1 & 0.4 & 52.4 & 1.0 \\
\hline 5 & 20.5 & 0.2 & 4.5 & 0.1 & 22.5 & 0.3 & 52.5 & 0.8 \\
\hline 6 & 20.9 & 0.2 & 4.4 & 0.2 & 22.3 & 0.3 & 52.4 & 0.8 \\
\hline 7 & 21.1 & 0.3 & 4.4 & 0.2 & 22.1 & 0.4 & 52.4 & 0.9 \\
\hline 8 & 27.7 & 0.3 & 12.6 & 0.3 & 9.1 & 0.3 & 50.7 & 0.9 \\
\hline 9 & 21.0 & 0.3 & 4.3 & 0.2 & 22.4 & 0.4 & 52.4 & 1.0 \\
\hline 10 & 21.9 & 0.2 & 4.5 & 0.1 & 21.4 & 0.3 & 52.2 & 0.7 \\
\hline 11 & 21.0 & 0.3 & 4.6 & 0.2 & 22.0 & 0.4 & 52.4 & 1.0 \\
\hline 12 & 20.5 & 0.3 & 4.5 & 0.2 & 22.5 & 0.4 & 52.5 & 1.0 \\
\hline 13 & 21.0 & 0.3 & 4.2 & 0.2 & 22.4 & 0.4 & 52.4 & 1.0 \\
\hline 14 & 21.6 & 0.3 & 4.0 & 0.2 & 22.1 & 0.4 & 52.3 & 1.0 \\
\hline 15 & 21.0 & 0.3 & 4.2 & 0.2 & 22.5 & 0.4 & 52.4 & 1.0 \\
\hline 16 & 27.9 & 0.3 & 12.7 & 0.3 & 8.8 & 0.3 & 50.6 & 1.0 \\
\hline
\end{tabular}

Figure S15: SEM of HP SAPO-34 and the corresponding EDXS data from the areas indicated showing that the composition is relatively uniform. 


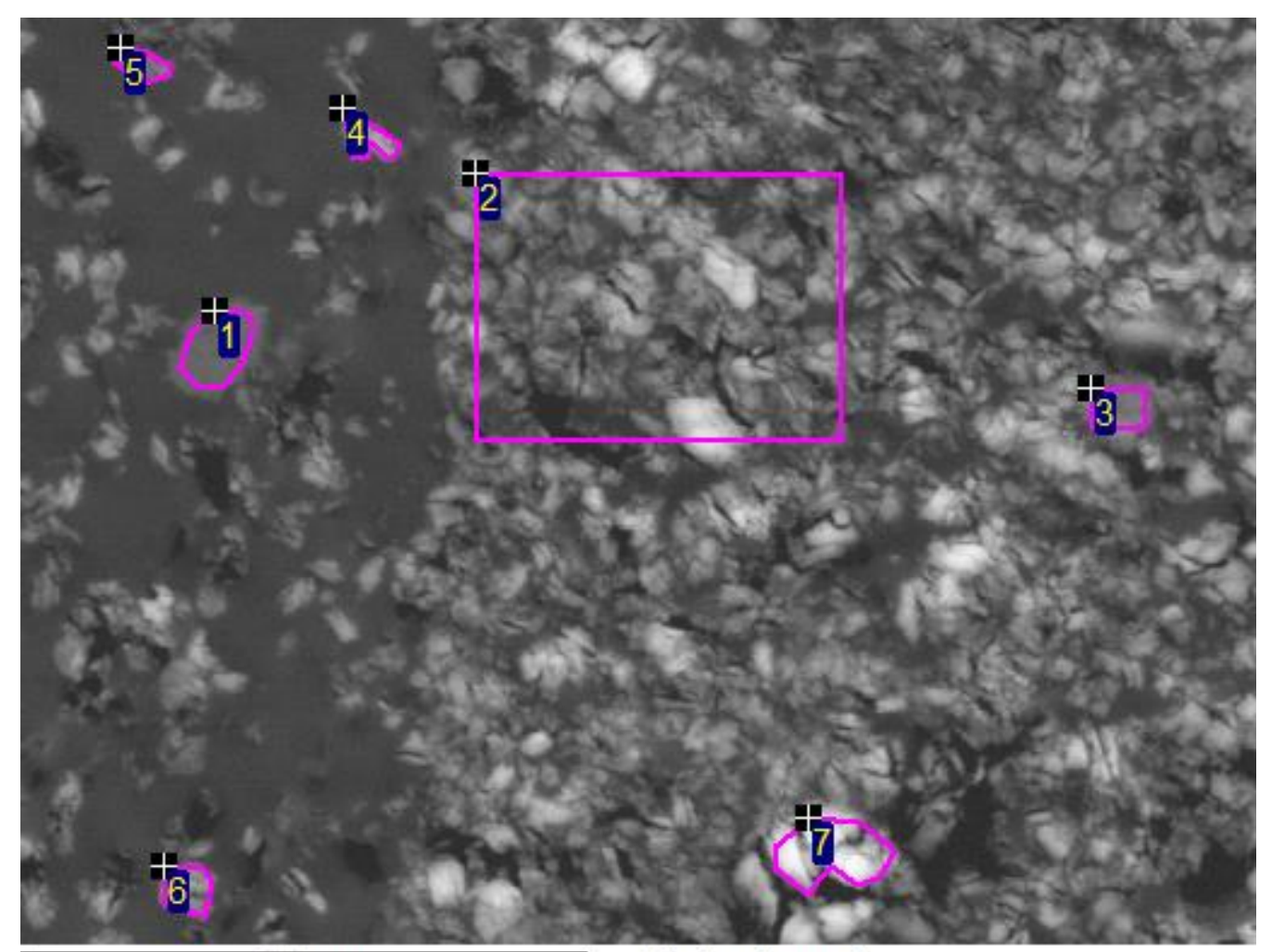

\begin{tabular}{|c|c|c|c|c|c|c|c|c|}
\hline \multicolumn{4}{|c|}{$30 \mu \mathrm{m}$} & \multicolumn{5}{|c|}{ Electron Image 1} \\
\hline Spec. & $\begin{array}{l}\text { wt\% } \\
\text { Al }\end{array}$ & $+/-$ & $\begin{array}{l}\text { wt\% } \\
\text { Si }\end{array}$ & $+/-$ & wt\% P & $+/-$ & $\begin{array}{l}\text { wt\% } \\
\text { O }\end{array}$ & $+/-$ \\
\hline 1 & 27.0 & 0.5 & 3.8 & 0.3 & 17.8 & 0.5 & 51.4 & 1.5 \\
\hline 2 & 21.1 & 0.4 & 5.6 & 0.3 & 21.0 & 0.5 & 52.3 & 1.3 \\
\hline 3 & 23.7 & 0.4 & 7.8 & 0.3 & 16.9 & 0.4 & 51.7 & 1.2 \\
\hline 4 & 20.5 & 0.3 & 2.4 & 0.2 & 24.5 & 0.4 & 52.3 & 1.0 \\
\hline 5 & 20.1 & 0.3 & 2.5 & 0.2 & 24.7 & 0.4 & 52.7 & 1.1 \\
\hline 6 & 20.5 & 0.3 & 2.5 & 0.2 & 24.4 & 0.4 & 52.6 & 1.0 \\
\hline 7 & 20.6 & 0.2 & 2.4 & 0.1 & 24.4 & 0.3 & 52.6 & 0.7 \\
\hline
\end{tabular}

Figure S16: SEM of HP SAPO-5 and the corresponding EDXS data from the indicated areas. 


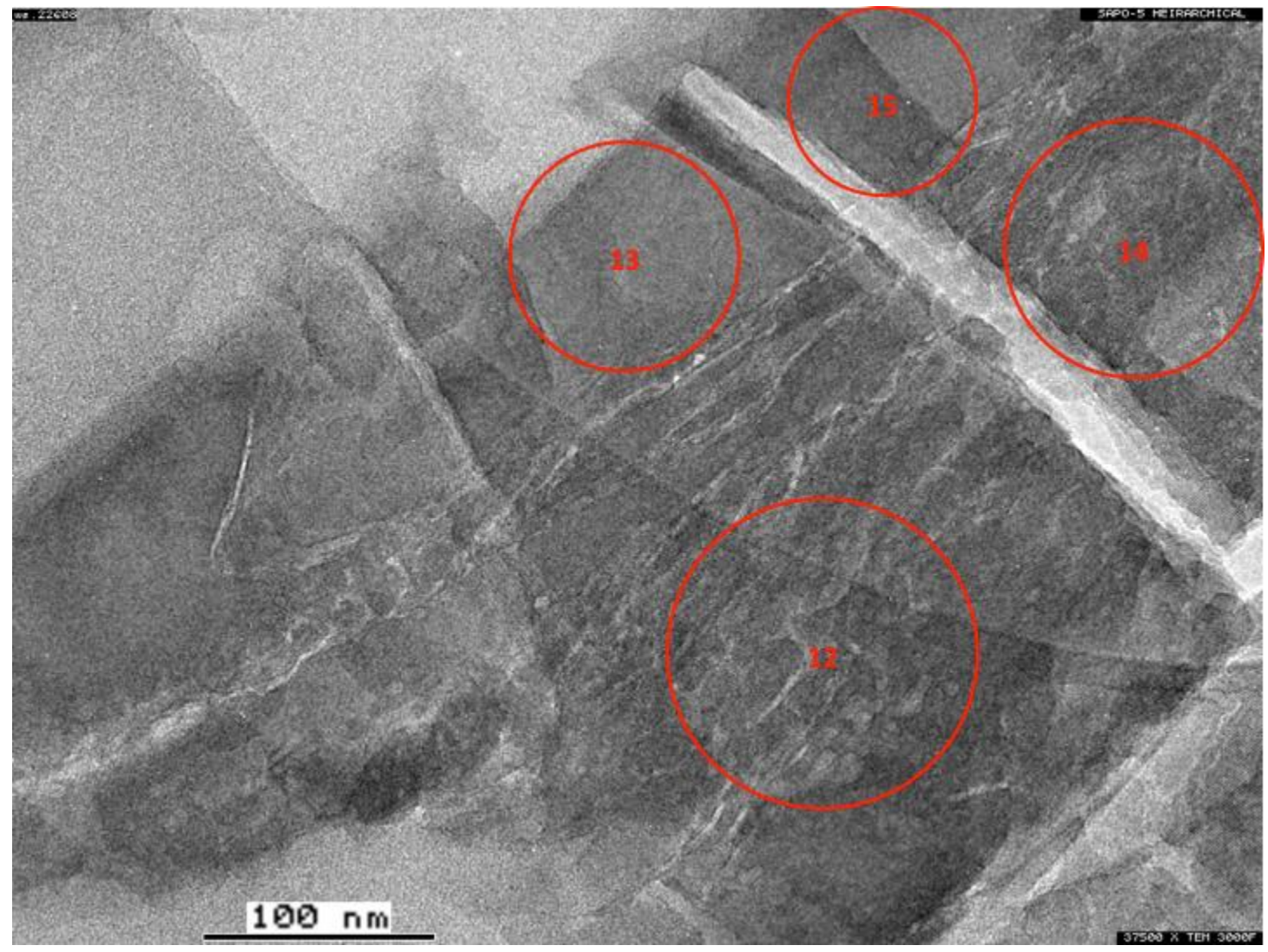

\begin{tabular}{|l|l|l|l|l|l|l|}
\hline Spec. & $\begin{array}{l}\text { wt\% } \\
\text { Al }\end{array}$ & + /- & $\begin{array}{l}\text { wt\% } \\
\text { Si }\end{array}$ & + /- & wt\% P & + /- \\
\hline $\mathbf{1 2}$ & 21.4 & 0.2 & 2.0 & 0.1 & 24.1 & 0.3 \\
\hline $\mathbf{1 3}$ & 20.9 & 0.2 & 1.2 & 0.1 & 25.3 & 0.3 \\
\hline $\mathbf{1 4}$ & 21.3 & 0.2 & 2.3 & 0.1 & 24.0 & 0.3 \\
\hline $\mathbf{1 5}$ & 21.2 & 0.2 & 1.0 & 0.1 & 25.2 & 0.3 \\
\hline
\end{tabular}

Figure S17: TEM image of HP SAPO-5 with regions of mesoporosity in the faulted regions. The elemental analysis (top left) from the numbered areas indicated show the expected composition for a SAPO. 


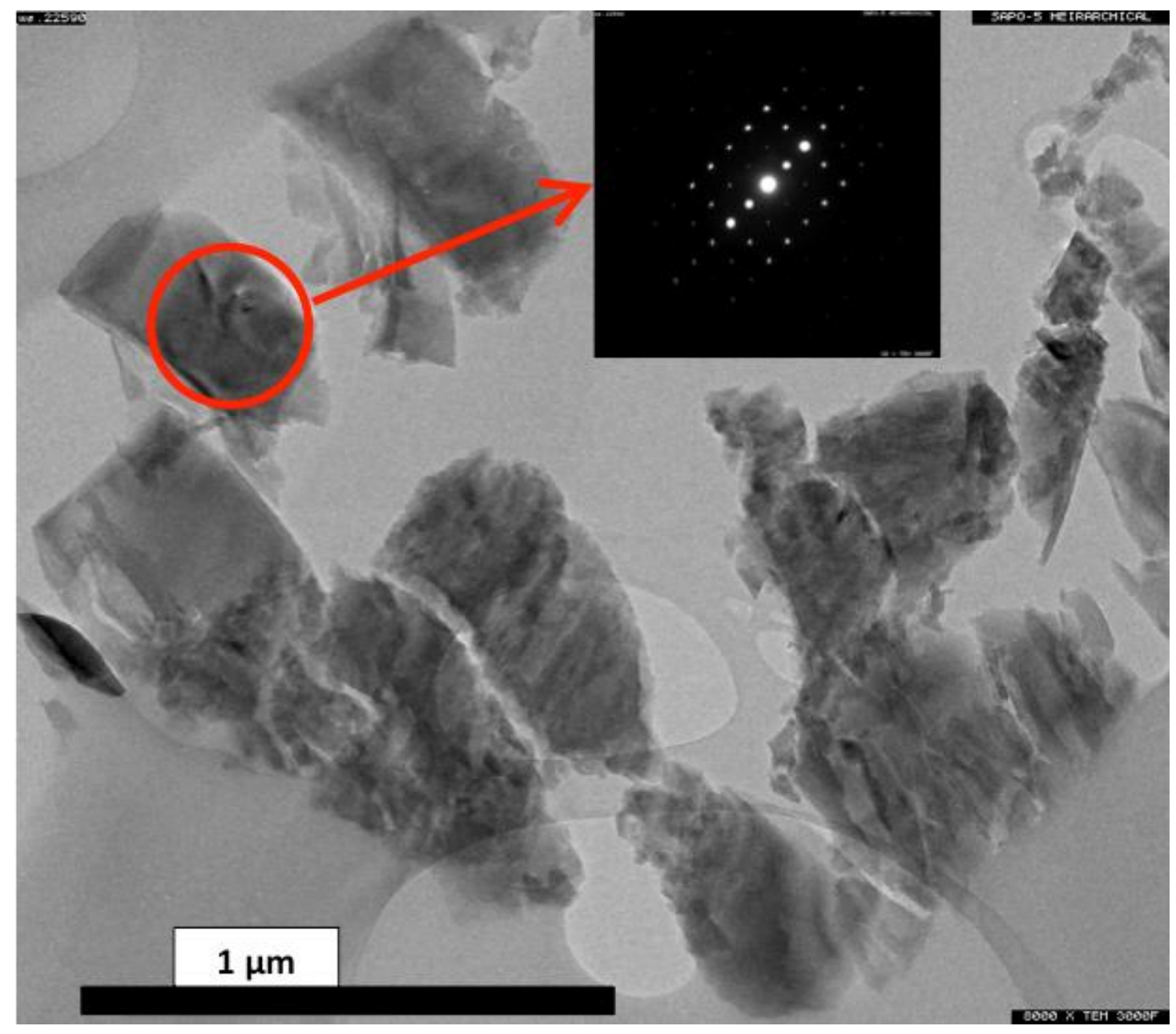

Figure S18: TEM image and associated selected area diffraction (inset) of HP SAPO-5 confirming that the areas of interest have the AFI topology. 


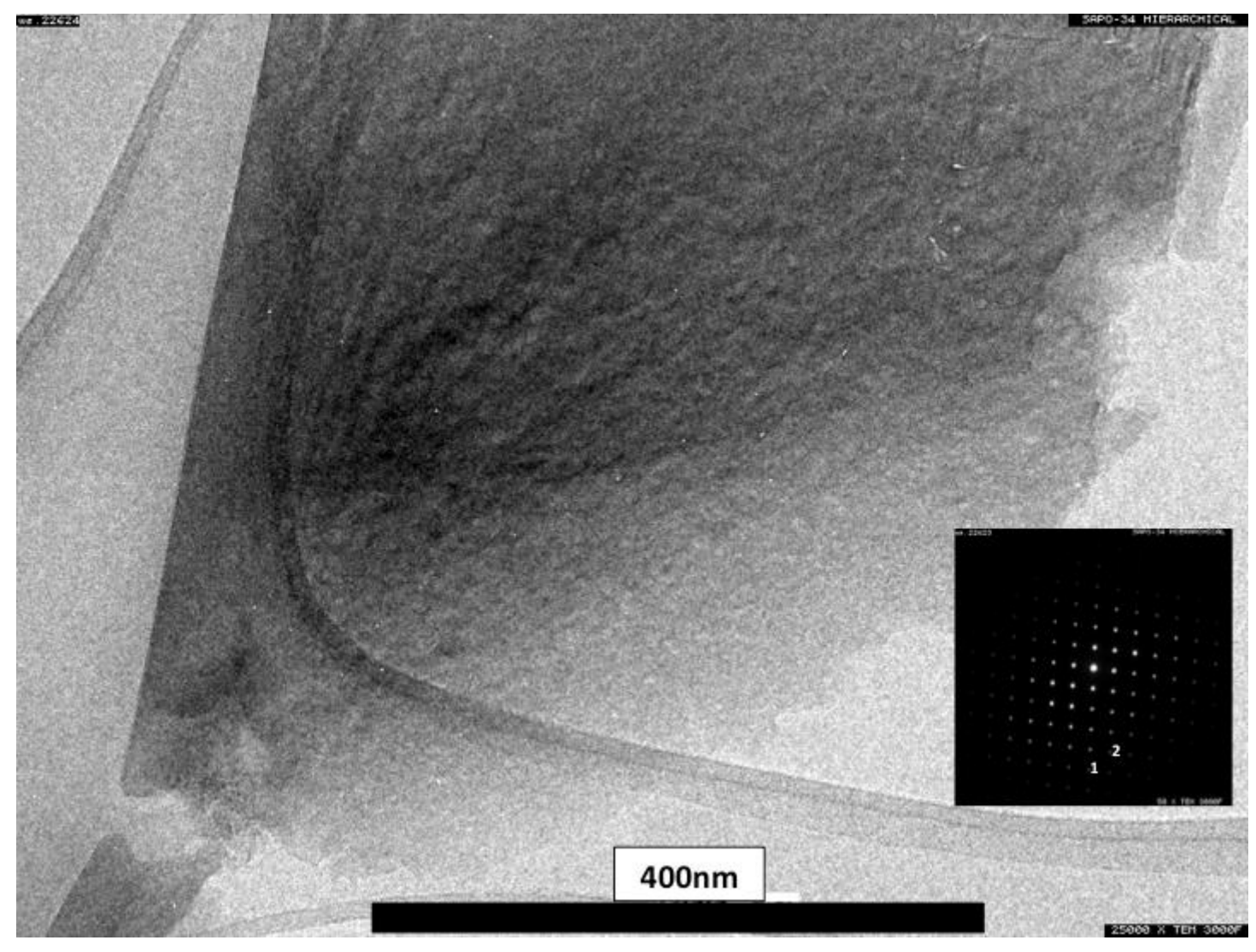

\begin{tabular}{|l|l|l|l|l|l|l|l|}
\hline $\begin{array}{l}\text { wt\% } \\
\text { Al }\end{array}$ & $+/-$ & $\begin{array}{l}\text { wt\% } \\
\text { Si }\end{array}$ & $+/-$ & wt\% P & $+/-$ & $\begin{array}{l}\text { wt\% } \\
\mathbf{O}\end{array}$ & $+/-$ \\
\hline 21.4 & 0.2 & 4.1 & 0.1 & 22.1 & 0.3 & 52.3 & 0.4 \\
\hline
\end{tabular}

Figure S19: TEM of HP SAPO-34 with confirmed CHA topology (inset, lower right) and the expected ratio of Al:Si:P. The two reflections (101) (inset lower right, spot 1 ) and $(-1,1,1)$ (inset lower right, spot 2) are equivalent to (100) and (101). The pores therefore appear to have rod-like morphology, elongated parallel to one or another of the rhombohedral basis vectors. 


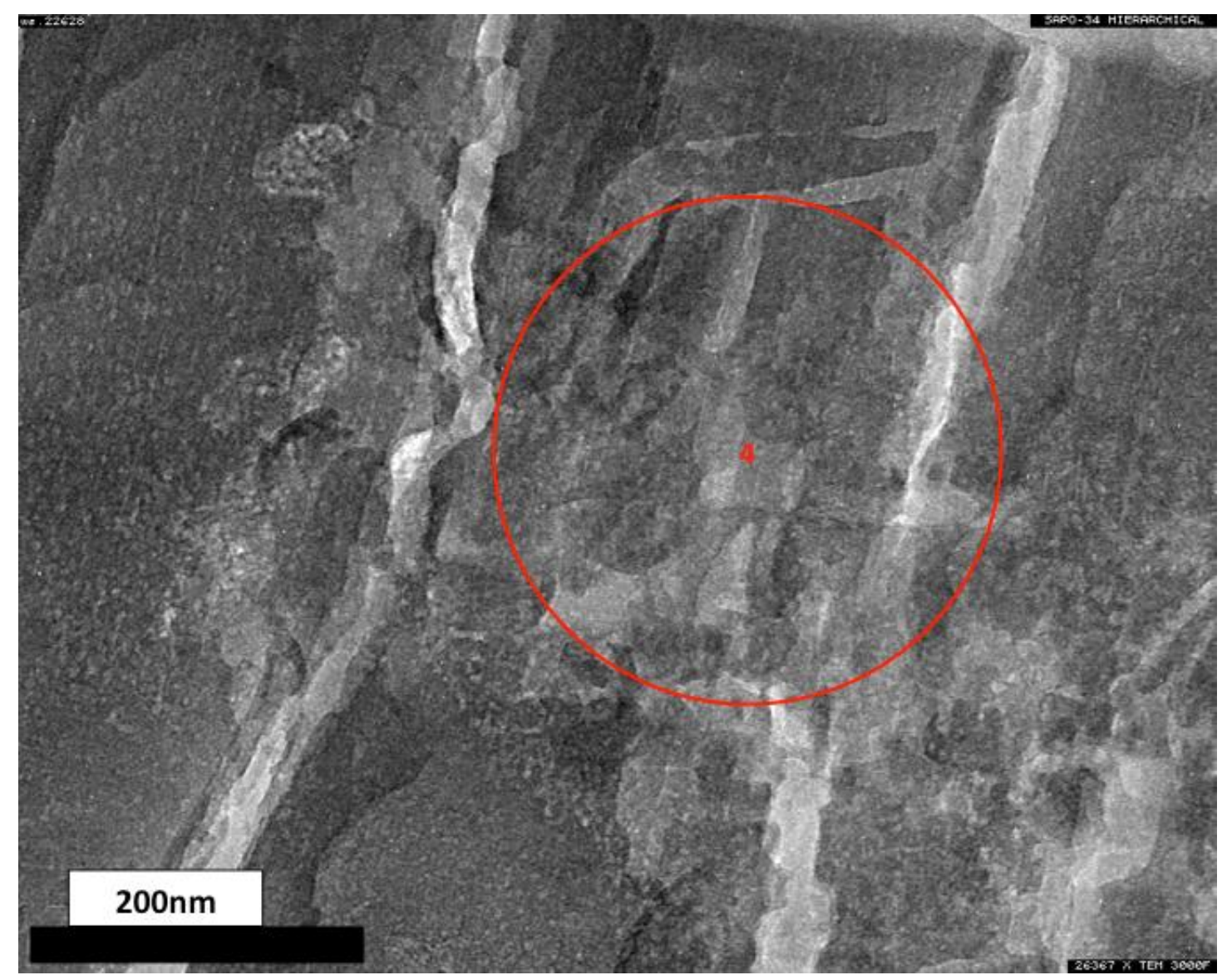

\begin{tabular}{|l|l|l|l|l|l|l|l|}
\hline $\begin{array}{l}\text { wt\% } \\
\text { Al }\end{array}$ & $+/-$ & $\begin{array}{l}\text { wt\% } \\
\text { Si }\end{array}$ & $+/-$ & wt\% P & $+/-$ & $\begin{array}{l}\text { wt\% } \\
\mathbf{O}\end{array}$ & $+/-$ \\
\hline 21.5 & 0.2 & 4.3 & 0.1 & 21.9 & 0.2 & 52.3 & 0.3 \\
\hline
\end{tabular}

Figure S20: TEM-EDXS of HP SAPO-34 showing the presence of some larger mesopores. The elemental concretion is from the area 4 , indicated. 


\section{Vapor-Phase Beckmann Rearrangement of Cyclohexanone Oxime}

Samples were analyzed after every hour when steady-state was achieved using a Clarus 480 gas chromatograph with FID detector and using an Elite 5 column. Products were identified against authenticated standards and quantified by calibration to obtain response factors $\left(\mathrm{R}_{\mathrm{F}}\right)$ against the known internal standard (mesitylene).

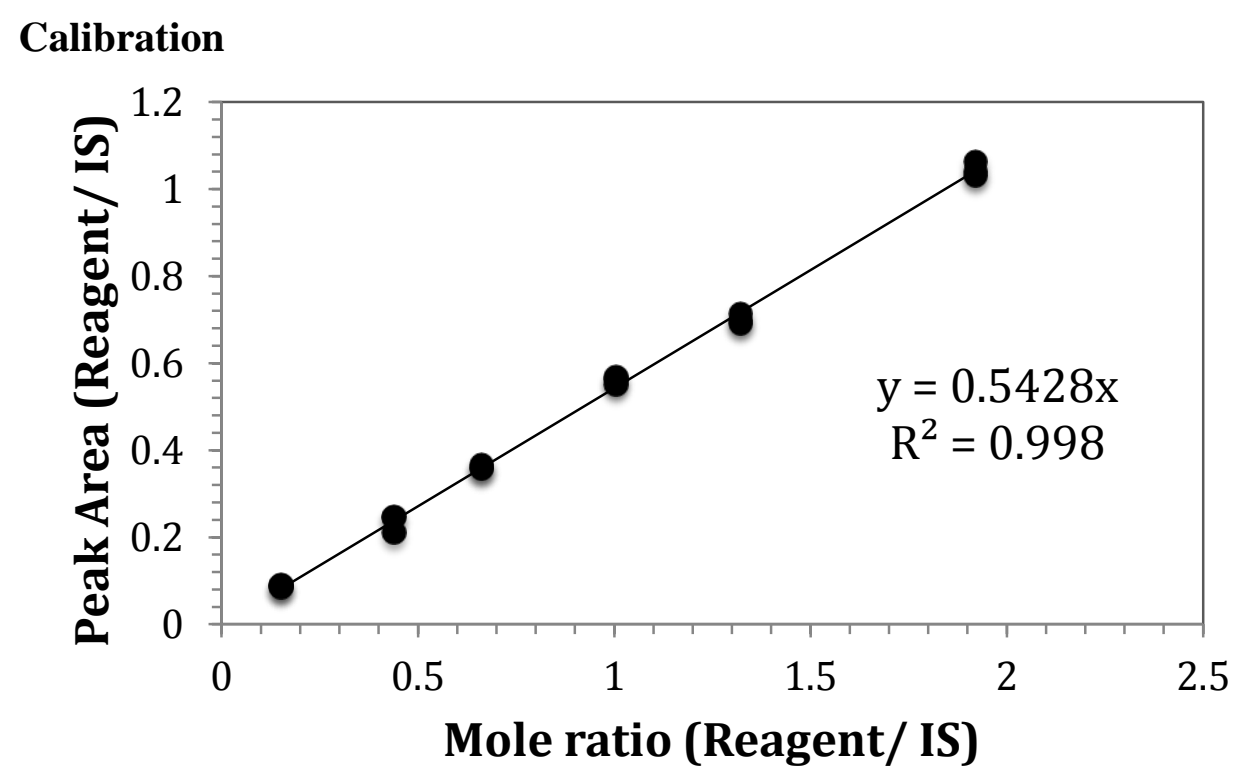

Figure S21: Calibration of cyclohexanone oxime against mesitylene (internal standard).

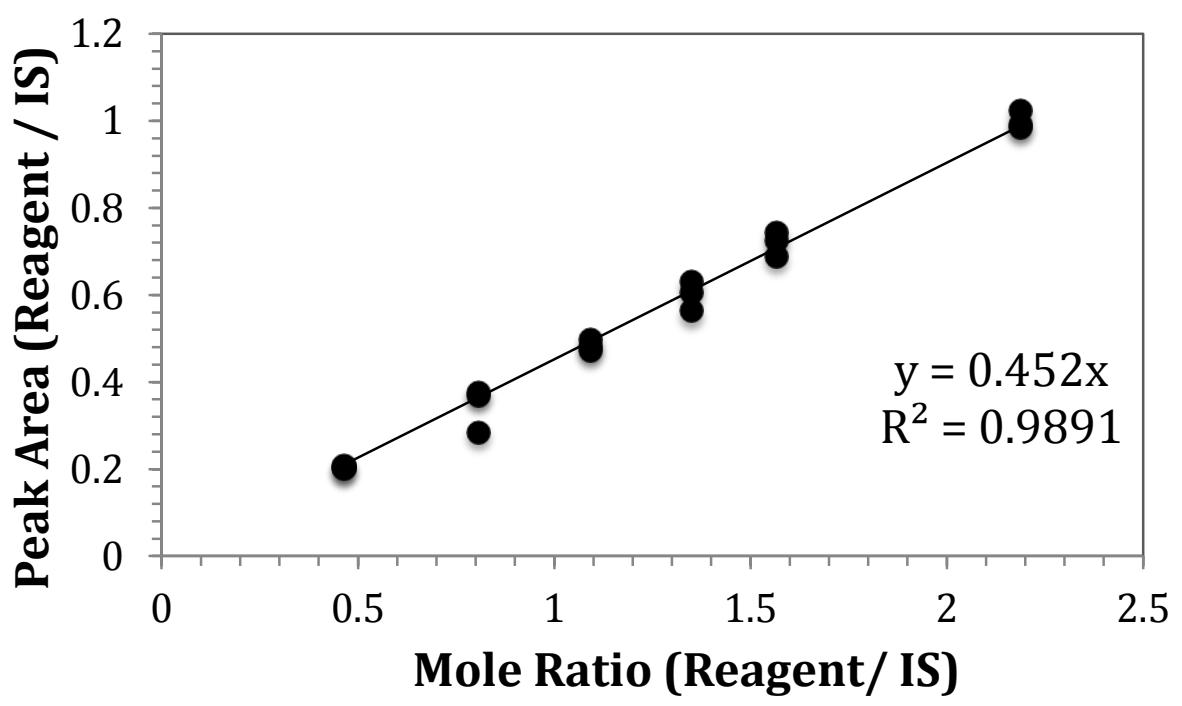

Figure S22: Calibration of $\varepsilon$ - caprolactam against mesitylene 


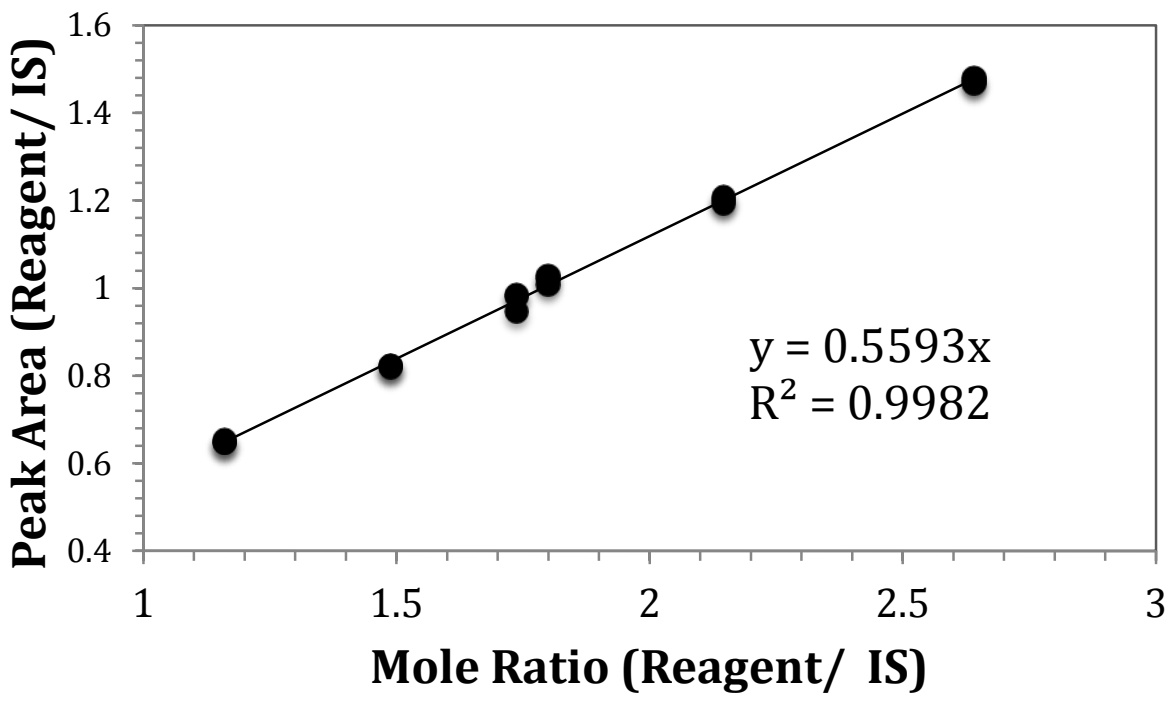

Figure S23: Calibration of cylcohexanone against mesitylene

\section{Mass Balance}

The feed solution for assessing the mass balance using mesitylene as the internal standard was composed of:

Mesitylene: $0.444 \mathrm{~g}$

Cyclohexanone oxime: $4.10 \mathrm{~g}$

Ethanol: $36.000 \mathrm{~g}$

Performing an identical procedure to the one described above, the following GC data was obtained at $598 \mathrm{~K}$, WHSV of $0.79 \mathrm{hr}^{-1}$ with HP SAPO-5 and by using the response factors it was possible to calculate the number of moles from the peak areas.

Table S7: Peak areas and calculated moles of the reagents in the catalytic reaction.

\begin{tabular}{|l|l|l|}
\hline Molecule & Peak Area (AU) & Calculated Moles \\
\hline Mesitylene & 124475 & 0.004 \\
\hline Cyclohexanone oxime & 0 & 0 \\
\hline Caprolactam & 393592 & 0.03 \\
\hline Cyclohexanone & 0 & 0 \\
\hline Others & 157437 & 0.01 \\
\hline
\end{tabular}

Conversion $=100 \times$ (Initial moles of oxime- Moles of oxime $) /$ Initial moles of oxime Conversion $=100 \%$

Selectivity $=100 \times($ Moles of lactam $) /$ Initial moles of oxime Selectivity $=\mathbf{7 3 . 6 \%}$ 
Mass balance $=100 \times($ (Moles of [oxime + lactam + ketone + others $]) /$ Initial moles of oxime)

Mass balance $=\mathbf{9 7 . 1 \%}$

Both the reproducibility and carbon balance are within accepted limits.

A blank reaction was performed with just the glass beads and after 6 hours at $598 \mathrm{~K}$ no conversion or production of caprolactam was detected.

\section{Selectivity Profiles}

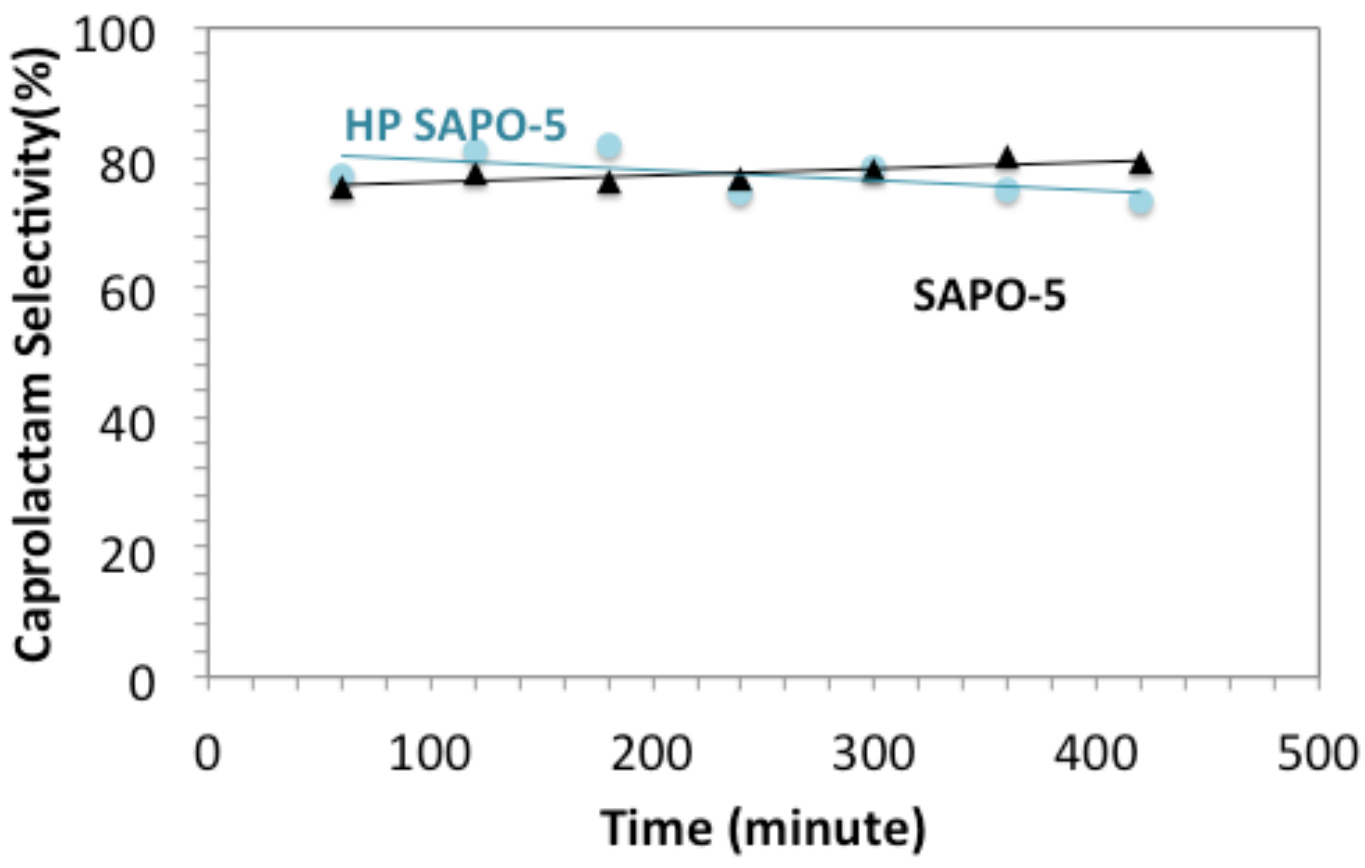

Figure S24: Selectivity towards $\varepsilon$ - caprolactam with HP SAPO-5 (blue) and SAPO5 (black) in the vapor-phase Beckmann rearrangement of cyclohexanone oxime. 


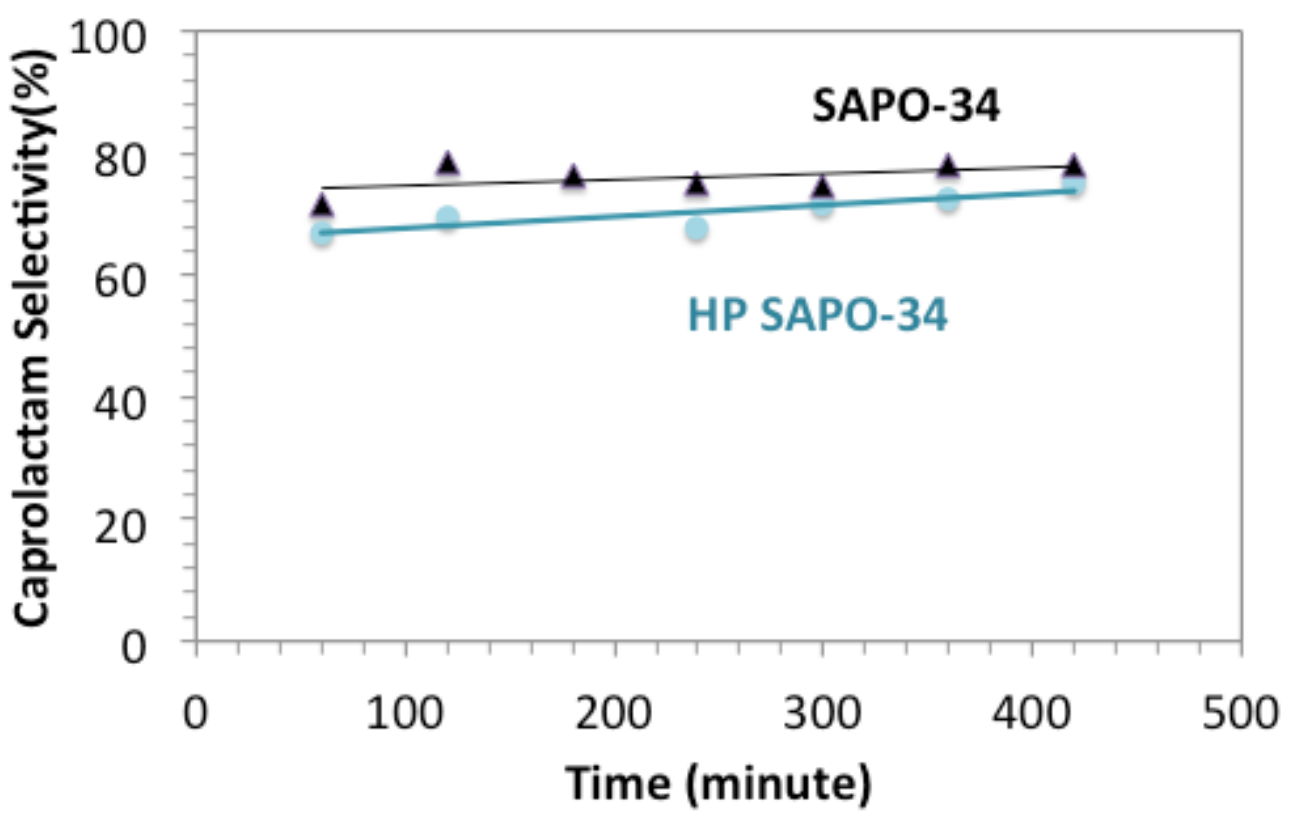

Figure S25: Selectivity towards $\varepsilon$ - caprolactam with HP SAPO-34 (blue) and SAPO-34(black) in the vapor-phase Beckmann rearrangement of cyclohexanone oxime.

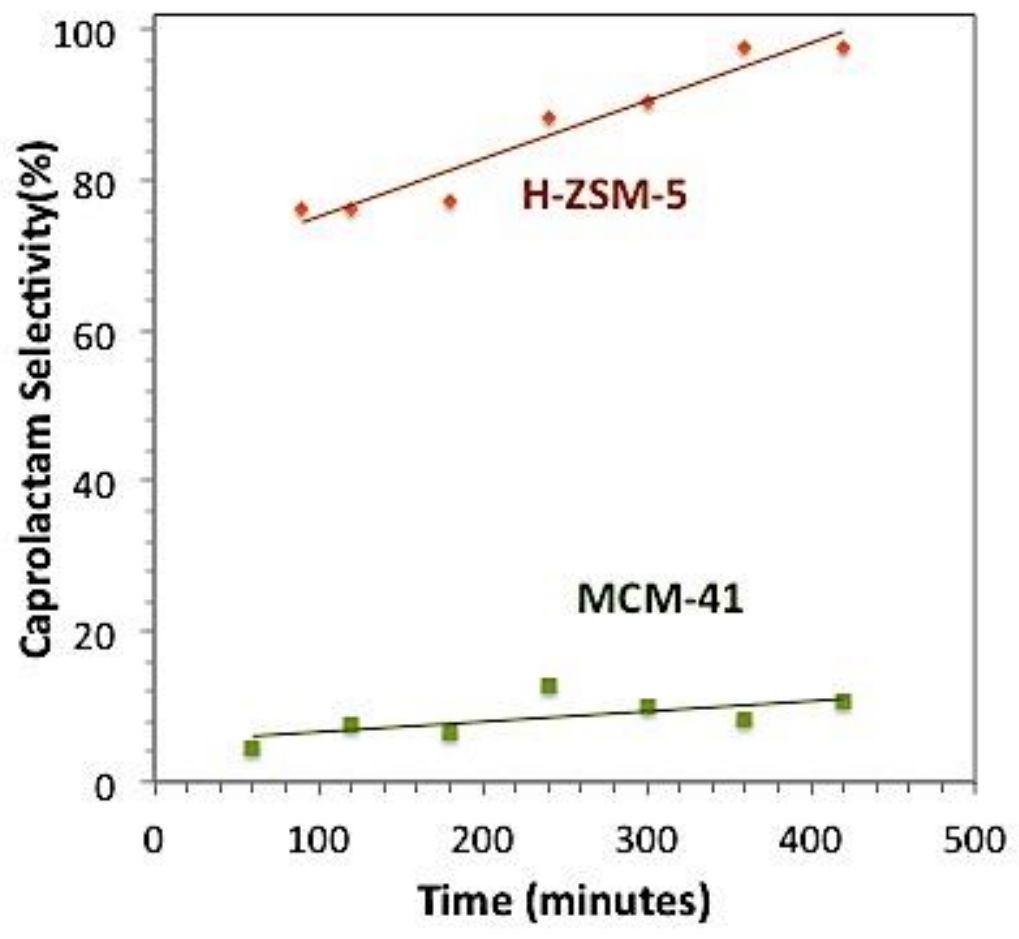

Figure S26: Selectivity towards $\varepsilon$ - caprolactam with H-ZSM-5 (orange) and MCM41(green) in the vapor-phase Beckmann rearrangement of cyclohexanone oxime. 
Spectroscopic characterization of the nature, type and strength of solidacid sites (sSNMR, FT-IR with molecular probes)

Solid State NMR

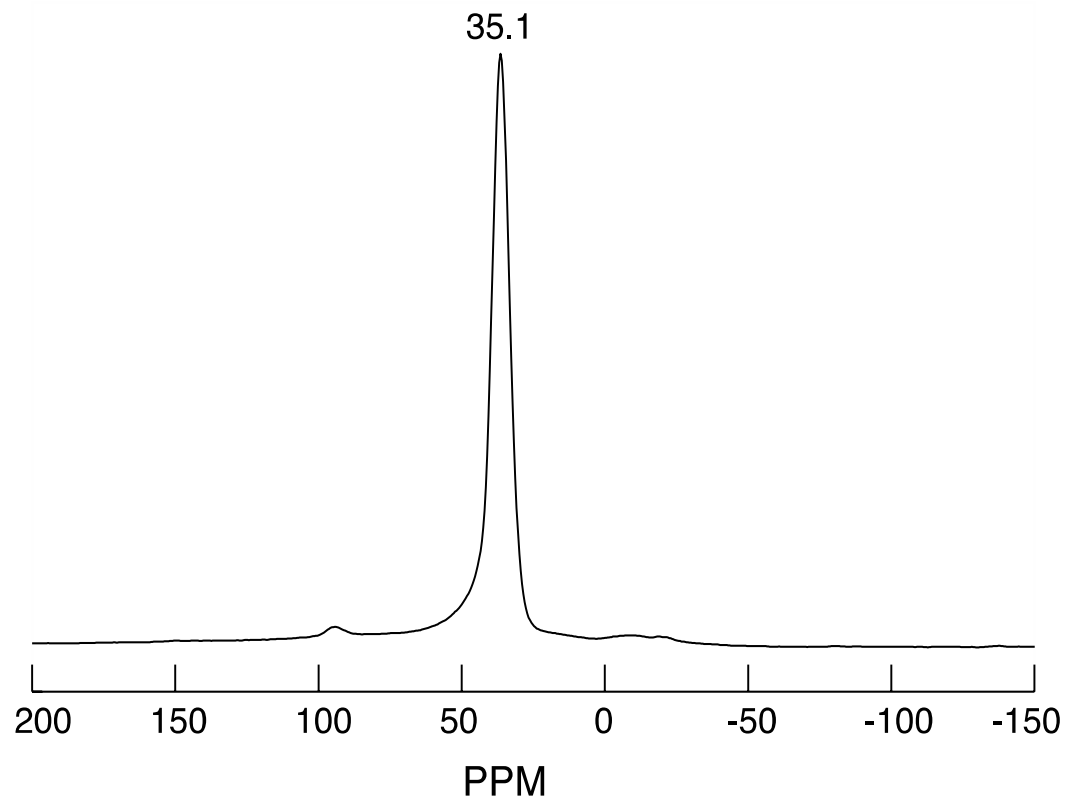

Figure S27: MAS ${ }^{27} \mathrm{Al}$ NMR of HP SAPO-5 indicating the presence of tetrahedral aluminium centres. Data acquired at $400 \mathrm{MHz}$ under MAS with $6 \mathrm{kHz}$ spinning frequency using 4 sec pulse delay.

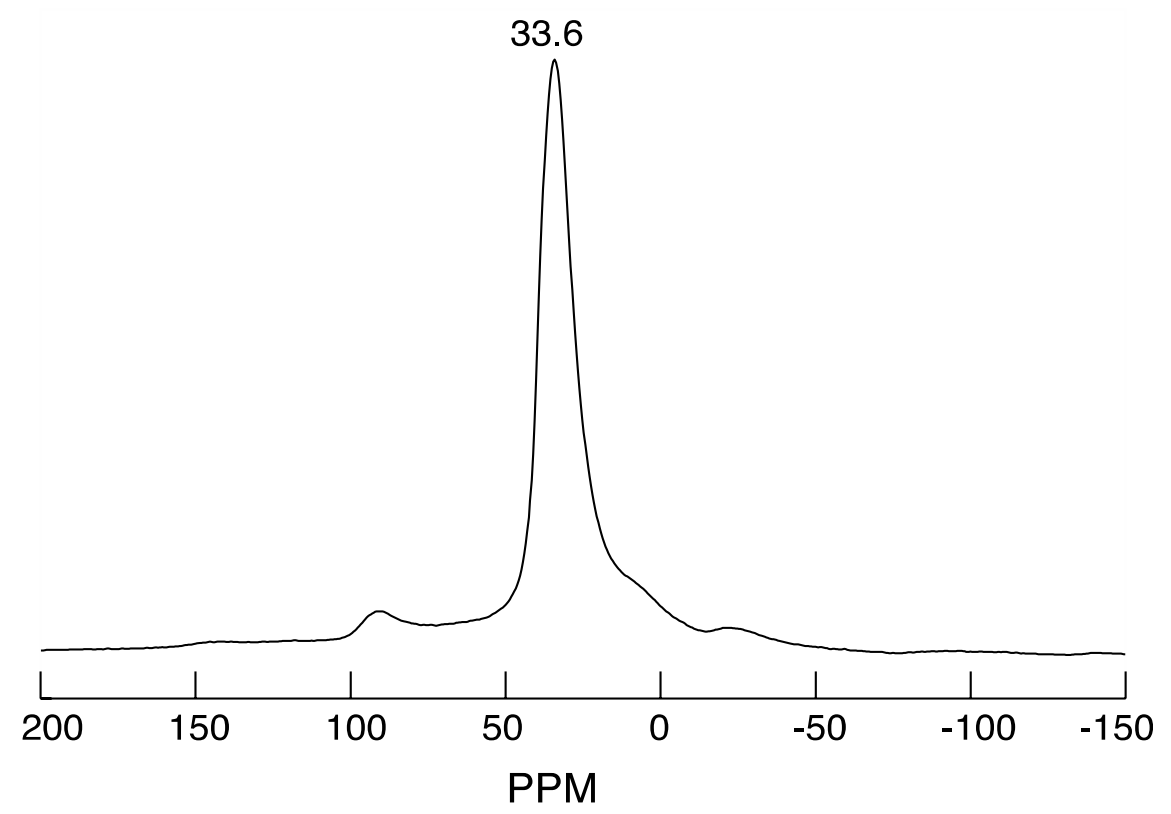

Figure S28: MAS ${ }^{27} \mathrm{Al}$ NMR of HP SAPO-34 indicating the presence of tetrahedral aluminium centres. Data acquired at $400 \mathrm{MHz}$ under MAS with $6 \mathrm{kHz}$ spinning frequency using 4 sec pulse delay. 


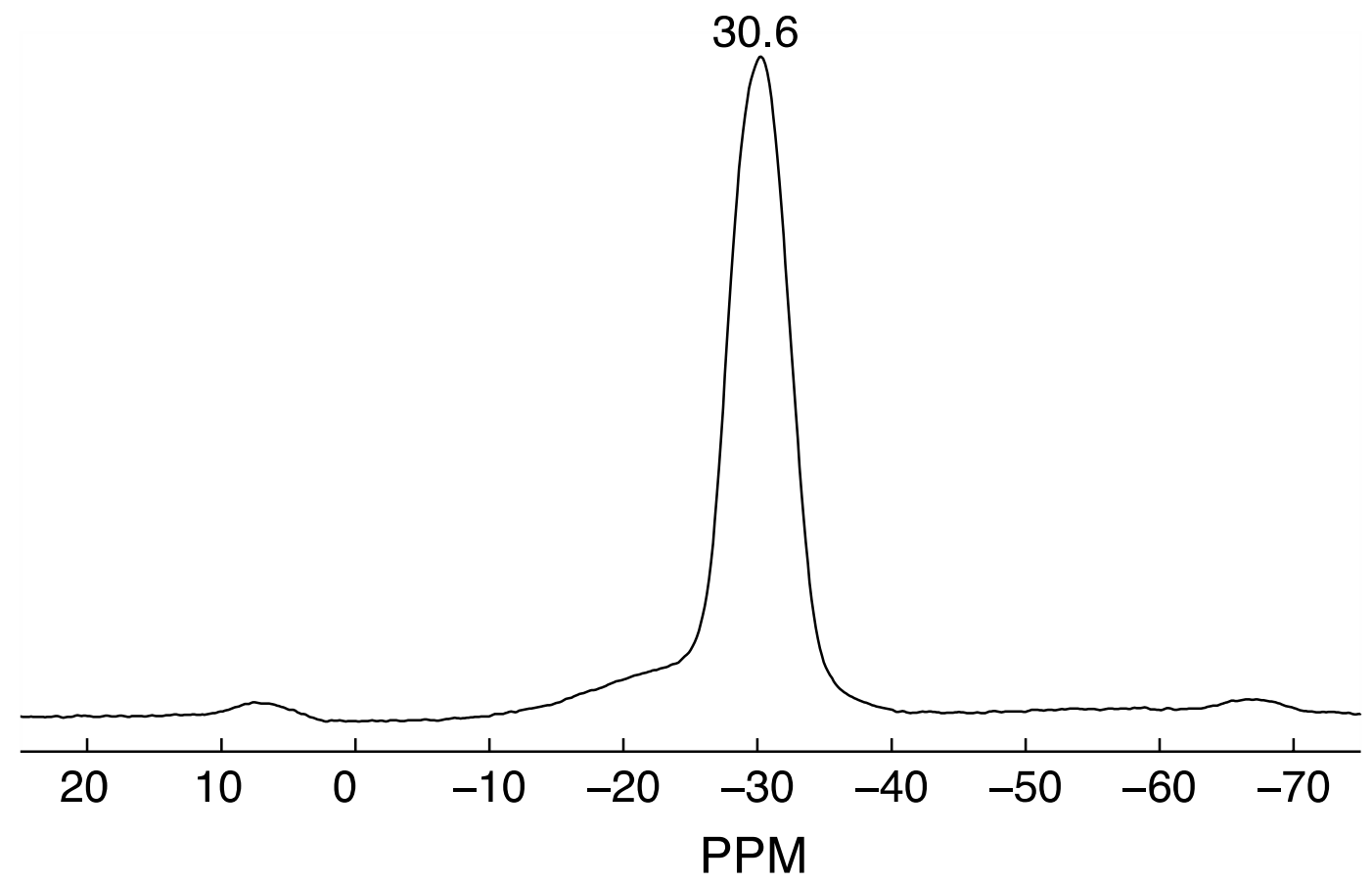

Figure S29: MAS 31P NMR of HP SAPO-5 indicating the presence of tetrahedral phosphorus centres. Data acquired at $400 \mathrm{MHz}$ under MAS with $6 \mathrm{kHz}$ spinning frequency using 3 minutes pulse delay.

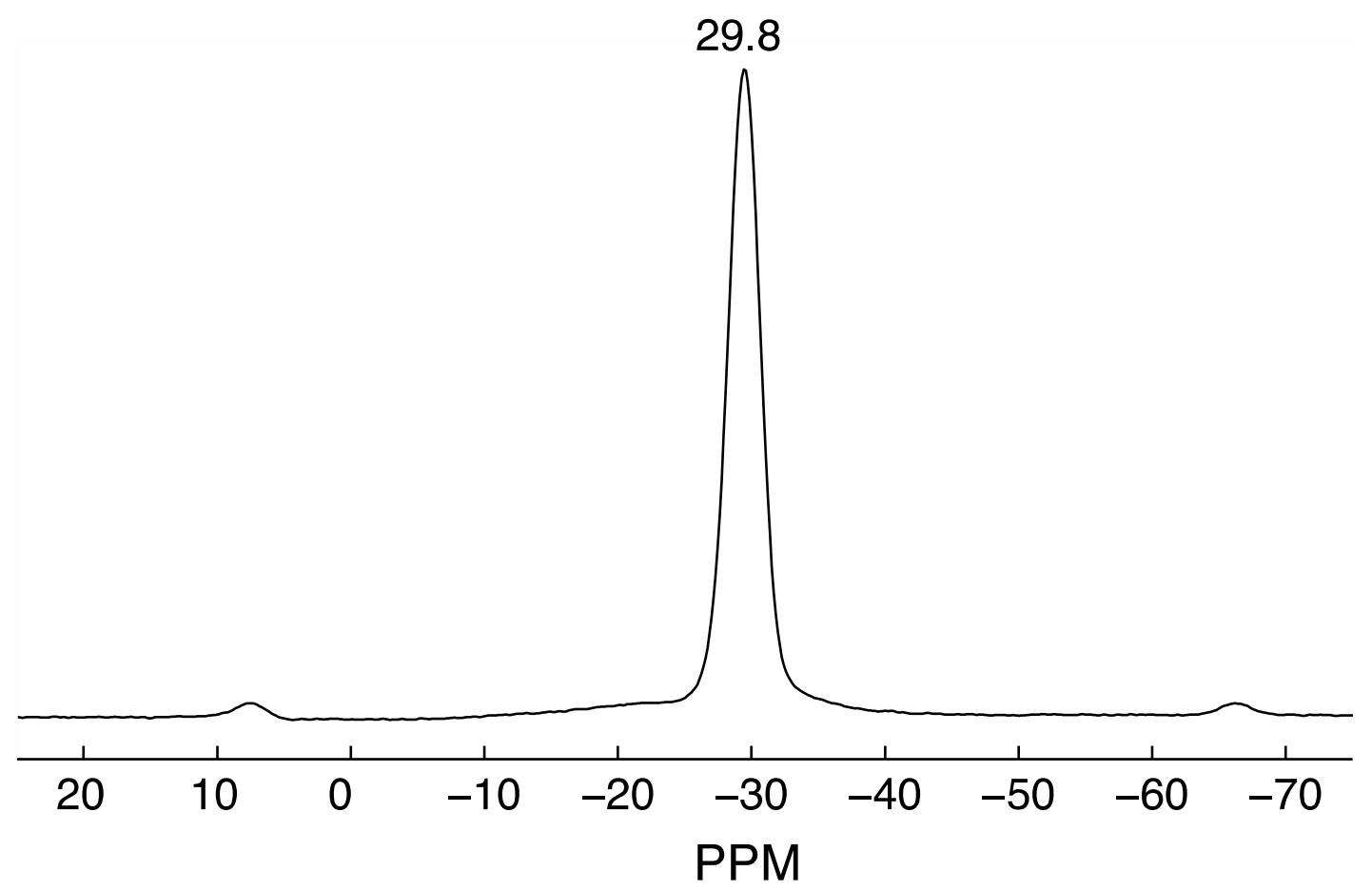

Figure S30: MAS ${ }^{31} \mathrm{P}$ NMR of HP SAPO-34 indicating the presence of tetrahedral phosphorus centres. Data acquired at $400 \mathrm{MHz}$ under MAS with $6 \mathrm{kHz}$ spinning frequency using 3 minutes pulse delay. 
FT-IR
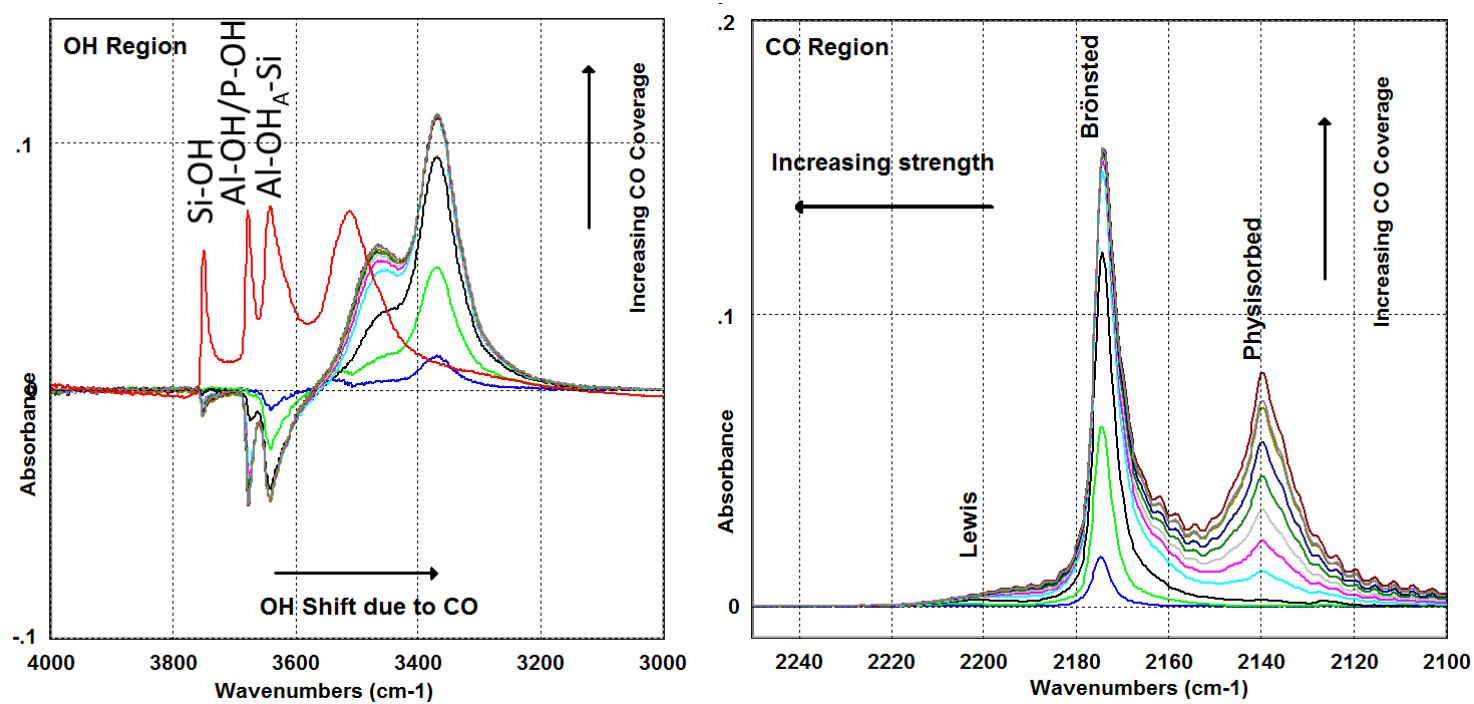

Figure S31: Low temp CO adsorption on HP SAPO-5
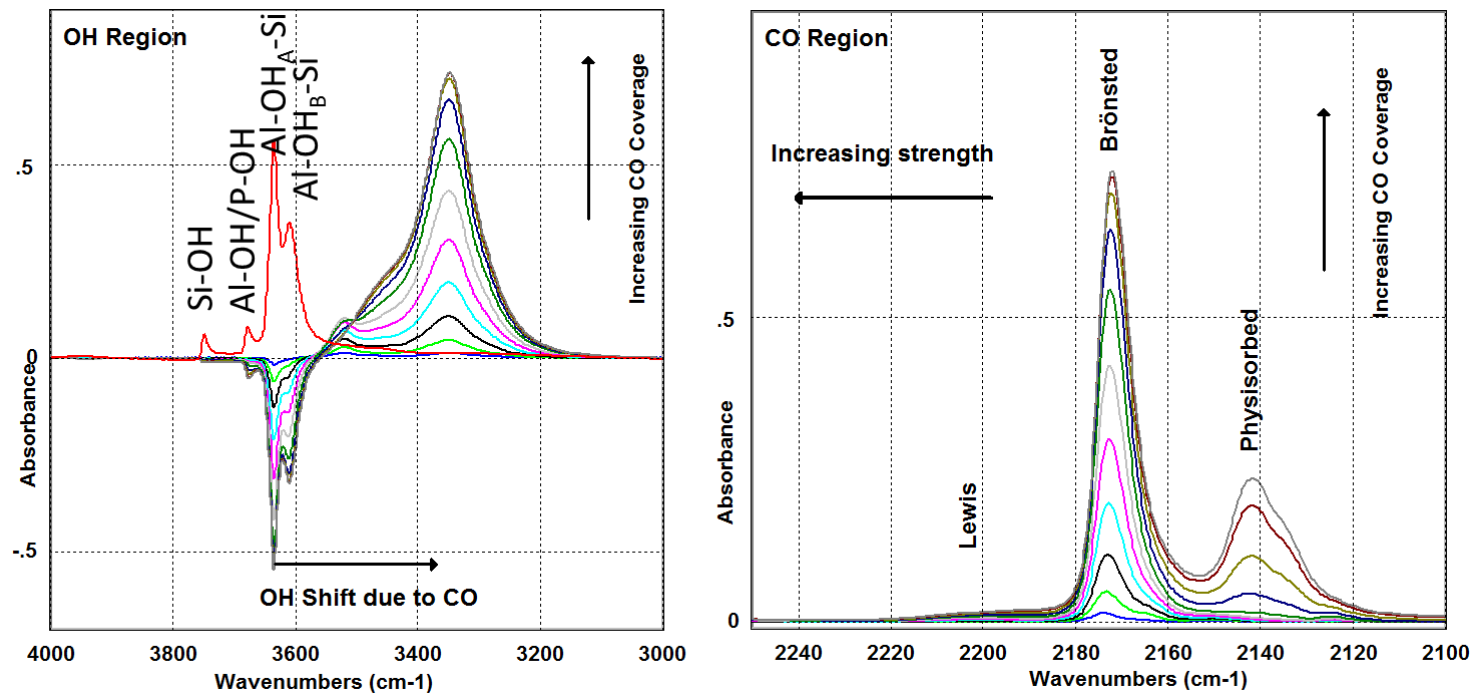

Figure S32: Low temp CO adsorption on HP SAPO-34 

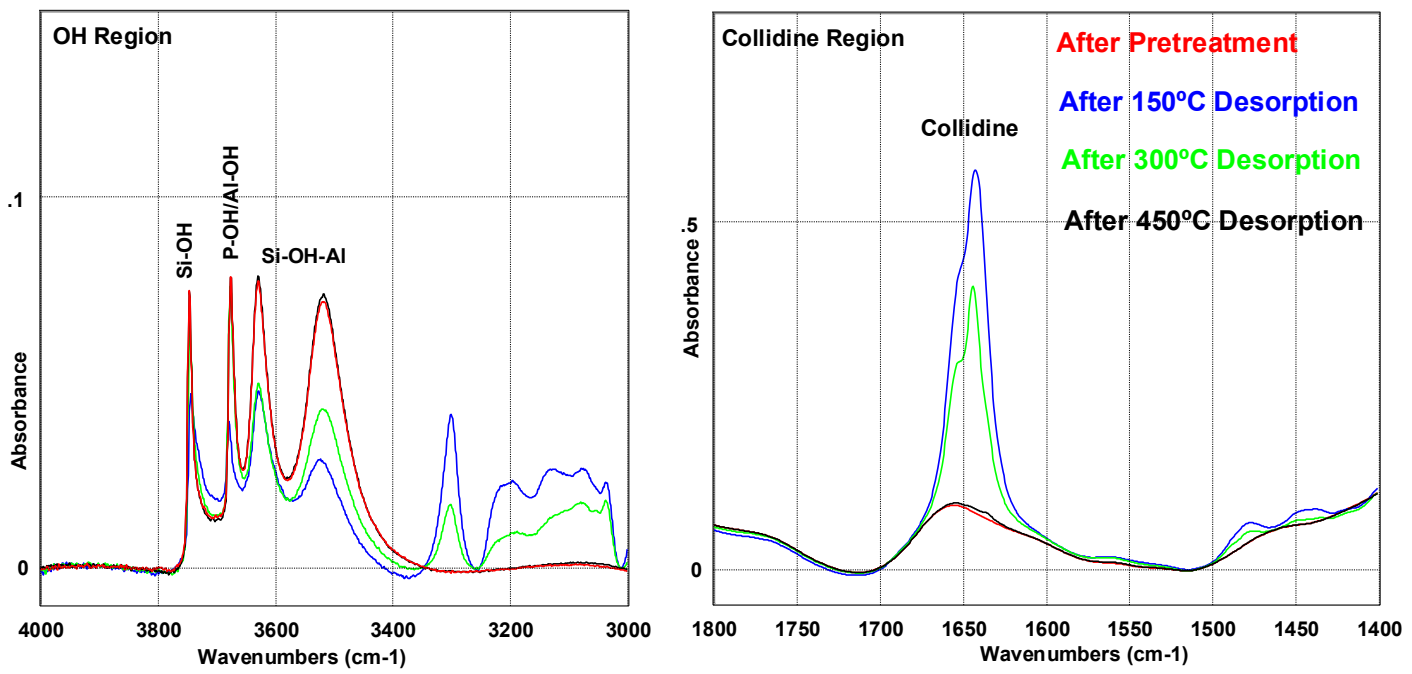

Figure S33: Collidine adsorption on HP SAPO-5. The collidine interacts with all of the $\mathrm{OH}$ group types after $150^{\circ} \mathrm{C}$ desorption. Essentially all the collidine is desorbed by $450^{\circ} \mathrm{C}$.
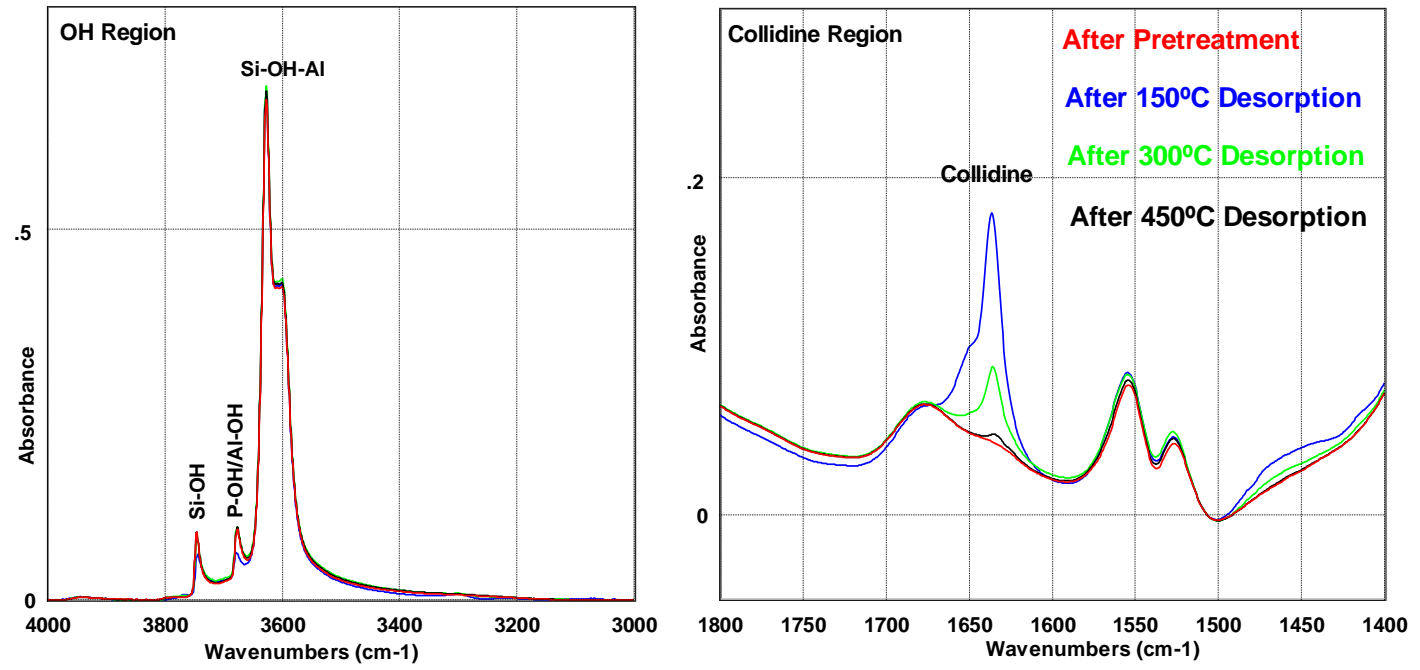

Figure S34: Collidine adsorption on HP SAPO-34. The collidine interacts primarily with the $\mathrm{Si}-\mathrm{OH}$ and $\mathrm{P}-\mathrm{OH}$ groups after $150^{\circ} \mathrm{C}$ desorption. 


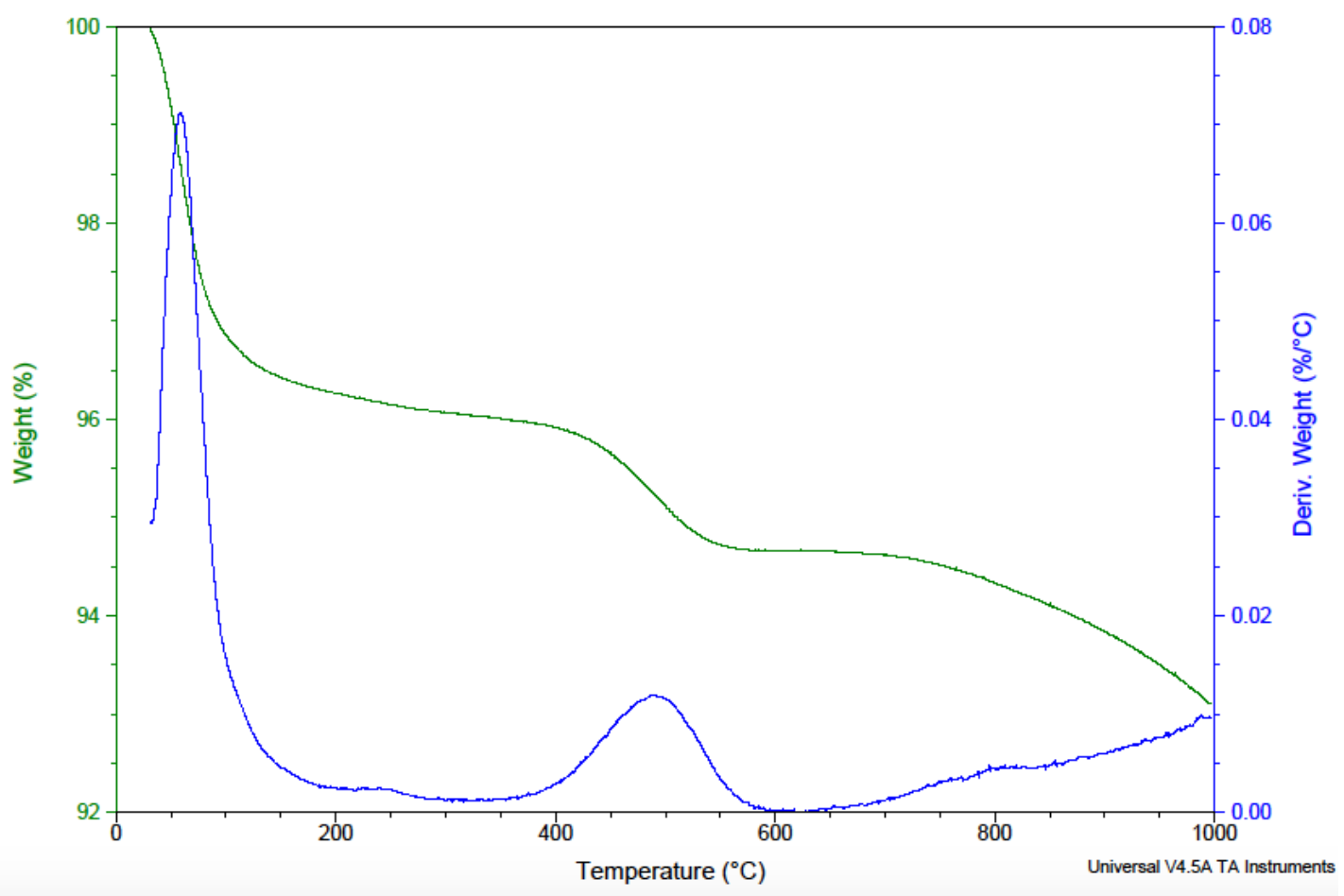

Figure S35: TGA analysis of used HP SAPO-5

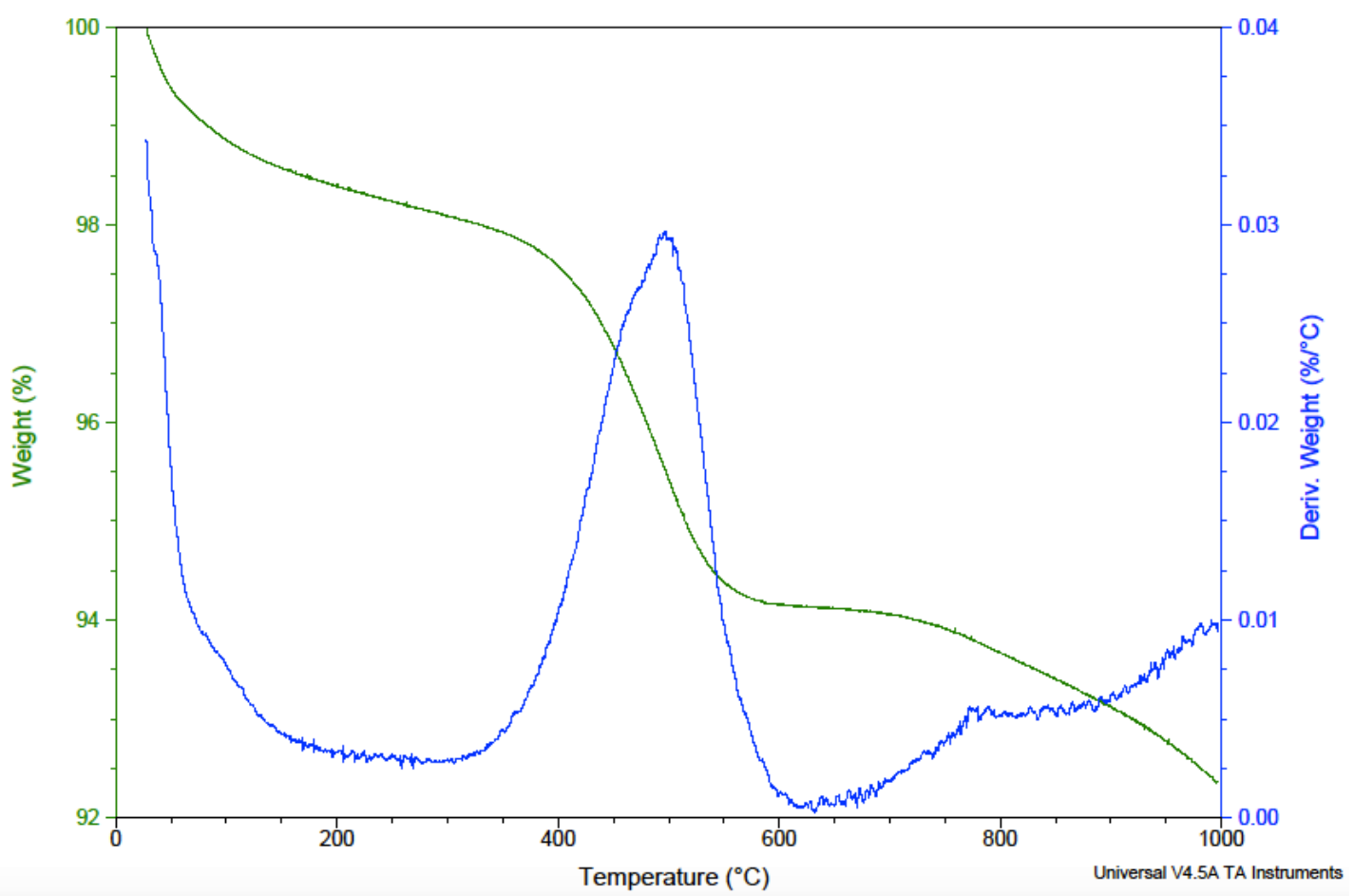

Figure S36: TGA analysis of used SAPO-5 


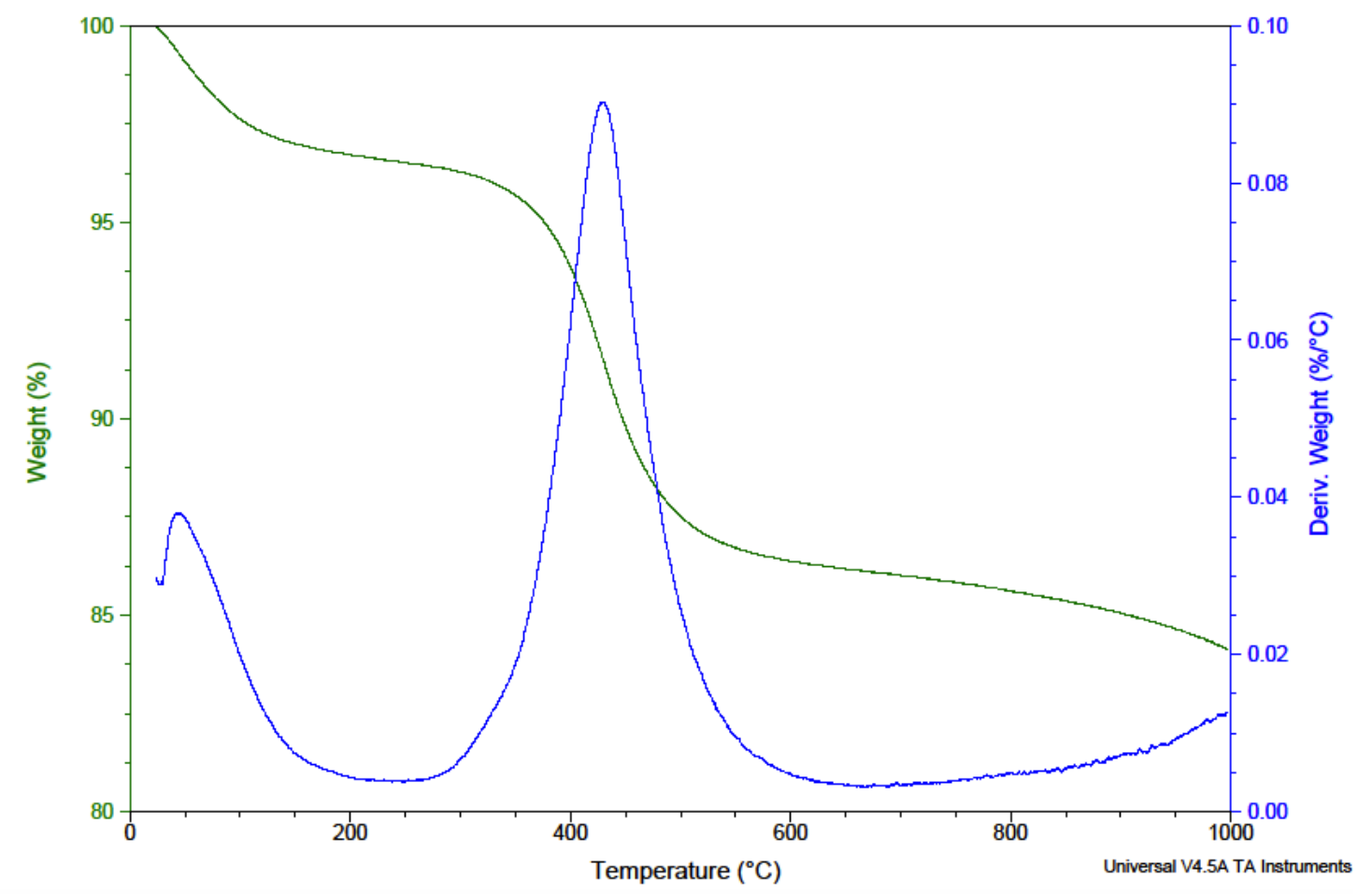

Figure S37: TGA analysis of used HP SAPO-34

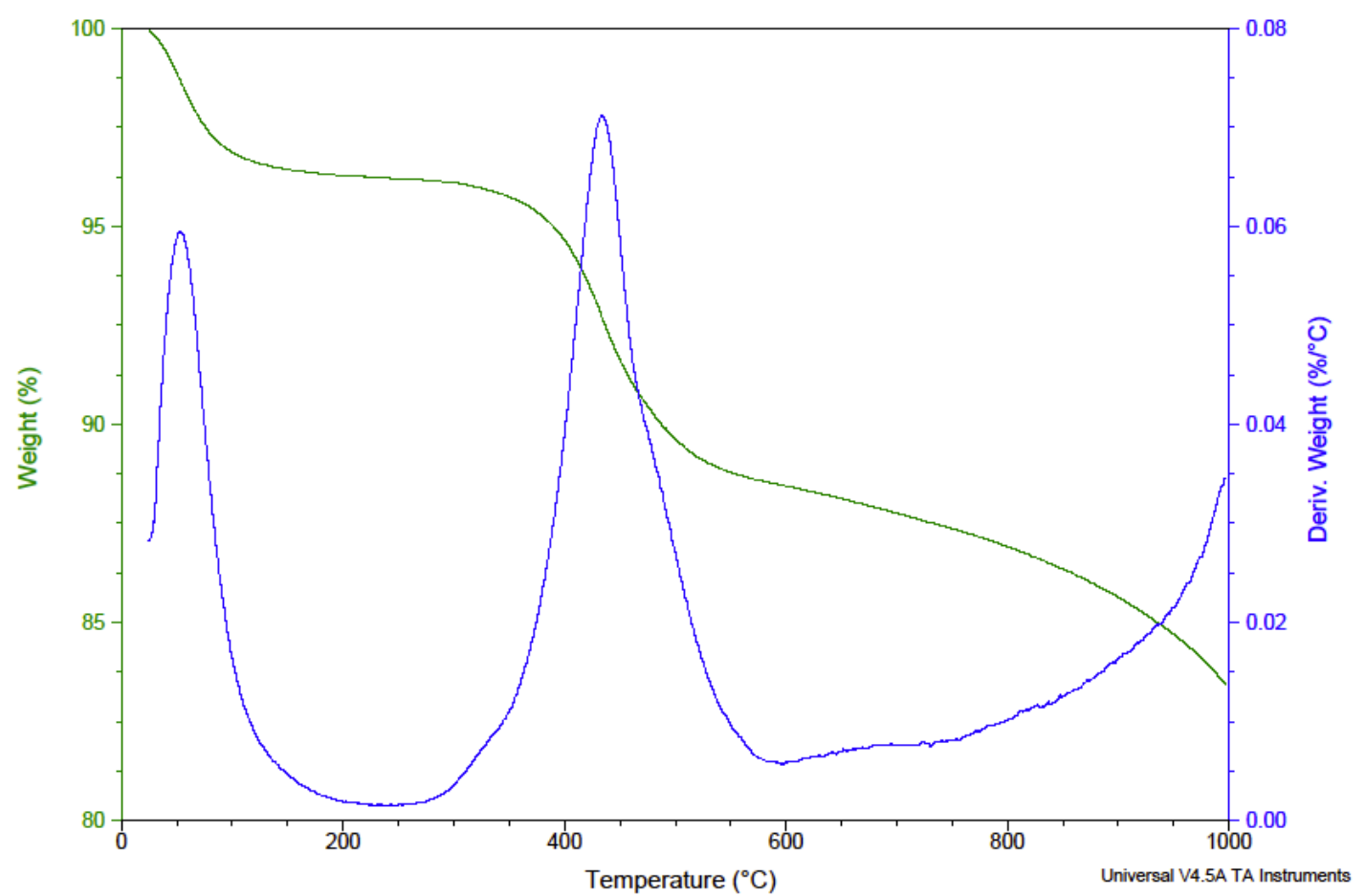

Figure S38: TGA analysis of used SAPO-34 


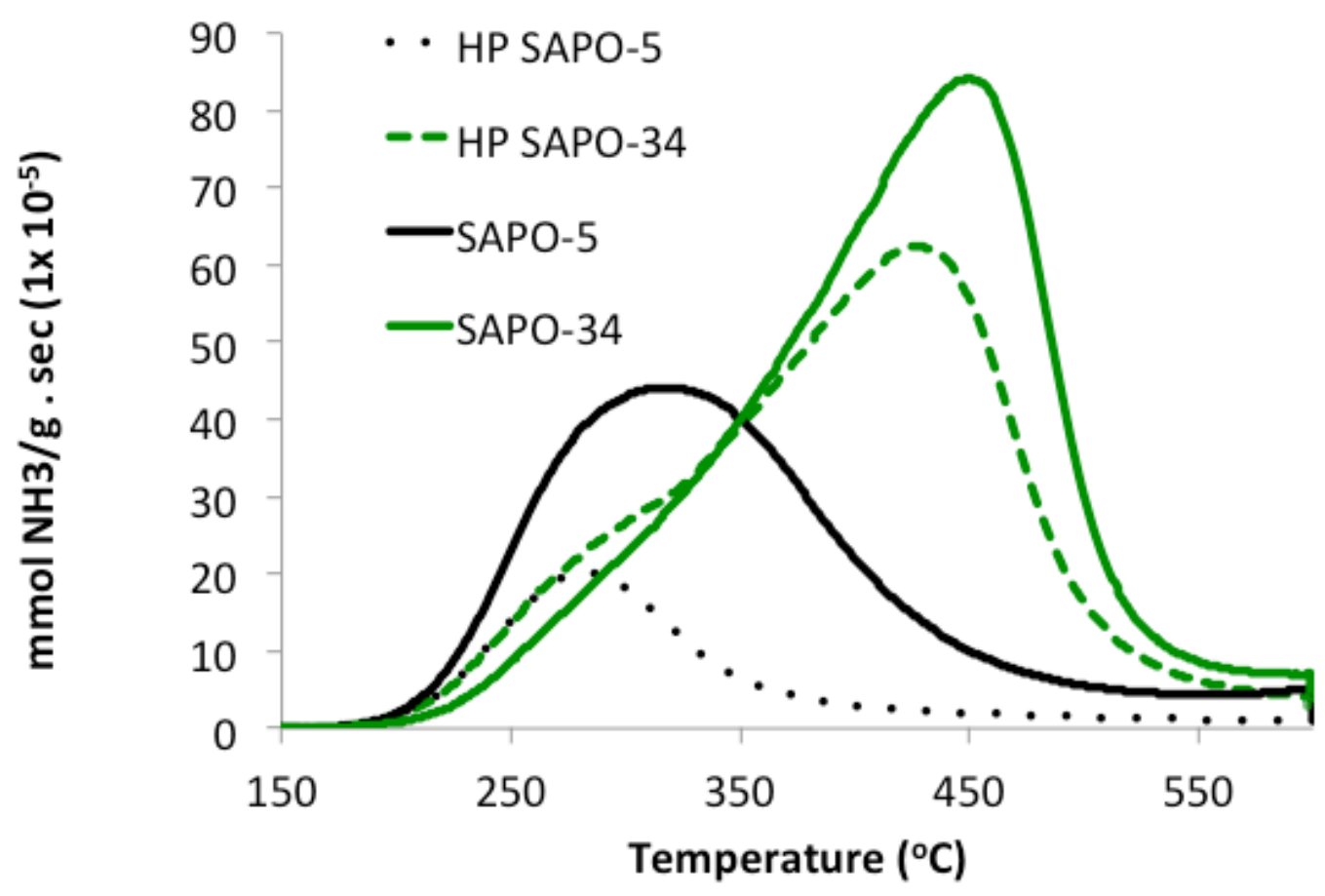

Figure S39: Temperature programme desorption of $\mathrm{NH}_{3}$ on the catalysts. The peak maxima are at similar temperatures for the hierarchically porous and the corresponding microporous catalysts indicating that the acid site strengths are comparable between the two systems. 


\section{Vapor-Phase Beckmann Rearrangement of Cyclooctanone Oxime}

\section{Mass Balance}

The feed solution for assessing the carbon balance using mesitylene as the internal standard was composed of:

Mesitylene: $0.4283 \mathrm{~g}$

Cyclooctanone oxime: $4.0288 \mathrm{~g}$

Ethanol: $35.7456 \mathrm{~g}$

Response factor for caprylic lactam: 0.7480 (Calibrated)

Response factor for cyclooctanone oxime: 0.6545 (Calibrated)

Response factor for cyclooctanone: 0.6781 (Calibrated)

Performing an identical procedure to the one described earlier for cyclohexanone oxime, the following GC data was obtained at 598K, WHSV of $0.79 \mathrm{hr}^{-1}$ with HP SAPO-5. By using the corresponding response factors it was possible to calculate the number of moles from the peak areas.

Table S8: Peak areas and calculated moles of the reagents in the catalytic reaction.

\begin{tabular}{|l|l|l|}
\hline Molecule & Peak Area (AU) & Calculated Moles \\
\hline Mesitylene & 56994 & 0.004 \\
\hline Cyclooctanone oxime & 10970 & 0.001 \\
\hline Caprylic lactam & 210061 & 0.020 \\
\hline Cyclooctanone & 0 & 0 \\
\hline Others & 9022 & 0.001 \\
\hline
\end{tabular}

Conversion $=100 \times$ (Initial moles of oxime- Moles of oxime $) /$ Initial moles of oxime Conversion $=95.9 \%$

Selectivity $=100 \times$ (Moles of lactam)/ Initial moles of oxime Selectivity $=94.3 \%$

Mass balance $=100 \times(($ Moles of [oxime+ lactam + ketone + others] $) /$ Initial moles of oxime)

Mass balance $=\mathbf{9 7 . 1 \%}$

Both the reproducibility and carbon balance are within accepted limits.

A blank reaction was performed with just the glass beads and after 6 hours at $598 \mathrm{~K}$ no conversion or production of caprylic lactam was detected. 


\section{Catalytic Activity}

\section{Selectivity}
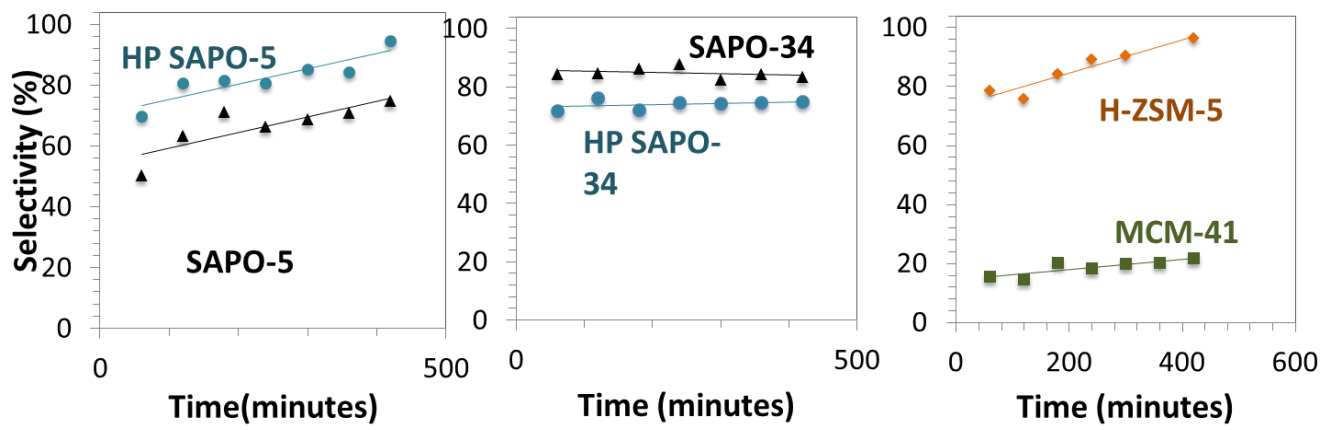

Figure S40: Selectivities for caprylic lactam in the vapor-phase Beckmann rearrangement of cyclooctanone oxime. 


\section{$\underline{\text { XRD Post Catalysis }}$}

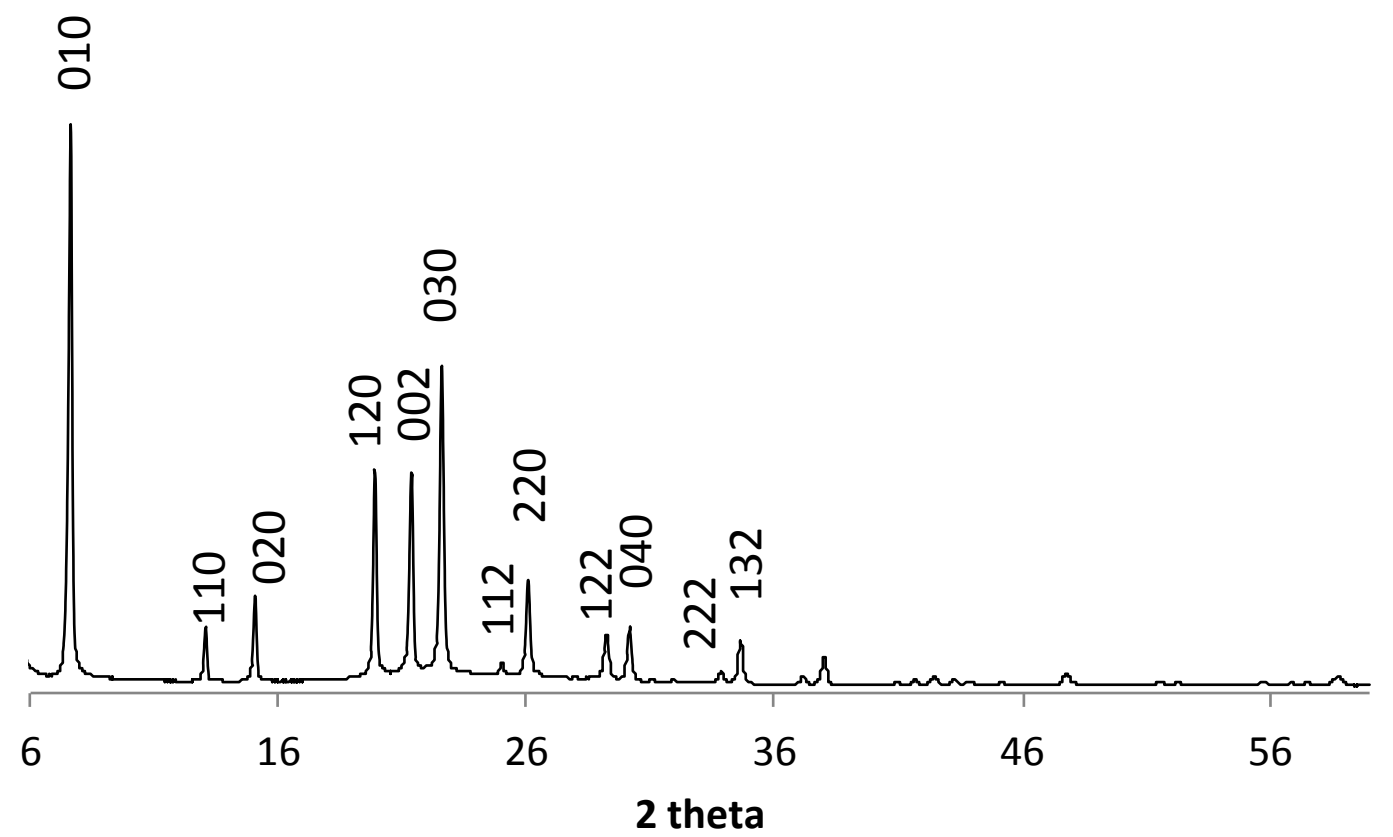

Figure S41: Typical XRD of HP SAPO-5 after catalysis indicating the structural integrity and phase-purity after reaction.

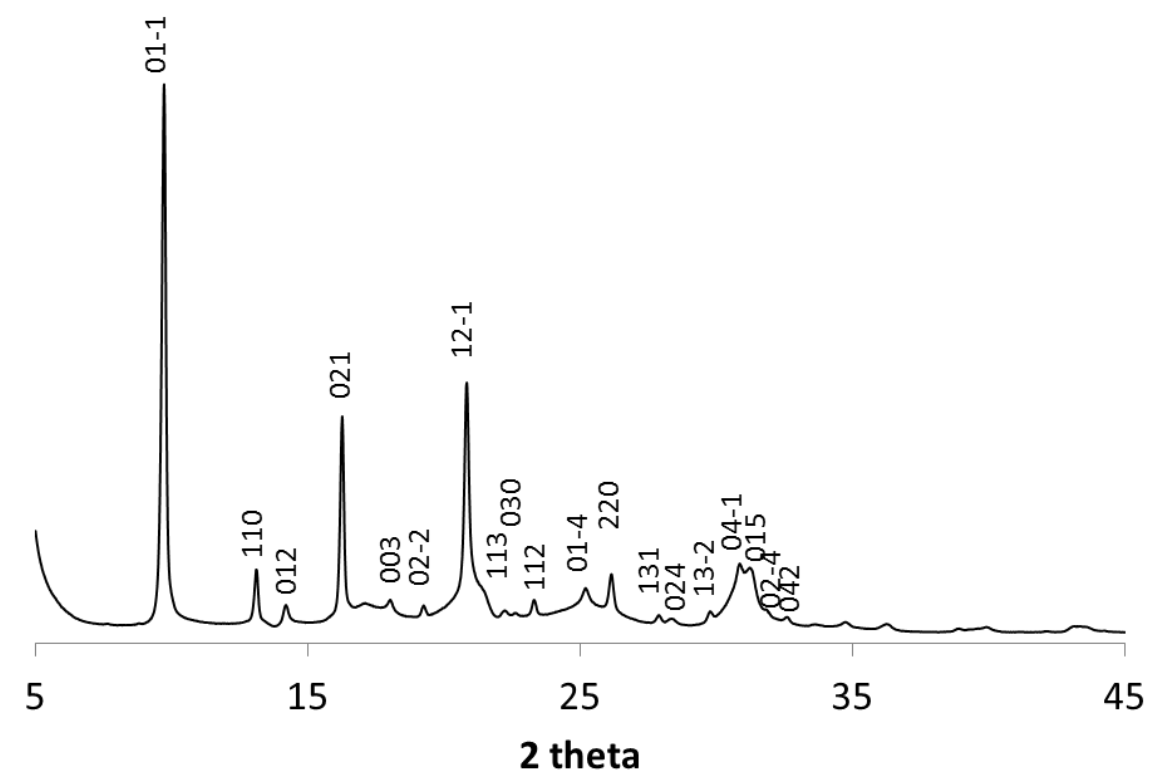

Figure S42: Typical XRD of HP SAPO-34 after catalysis indicating that the catalyst is phase-pure and stable after the reaction. 


\section{$\underline{\text { References }}$}

1. Lang, N; Tuel, A. Chem. Mater. 2004, 16, 1961-1966 